\title{
The effect of dimethyl fumarate on phenotype and function of B lymphocytes in MS patients
}

\author{
INAUGURAL-DISSERTATION \\ zur Erlangung des Doktorgrades \\ der Medizinischen Fakultät der \\ Georg-August-Universität zu Göttingen
}

\author{
vorgelegt von \\ Jan Werner Traub \\ aus
}

Bad Urach

Göttingen 2020 
Dekan:

\section{Betreuungsausschuss:}

Betreuer:

Ko-Betreuer:

\section{Prüfungskommission:}

Referent:

Ko-Referent:

Datum der mündlichen Prüfung:
Prof. Dr. med. W. Brück

Prof. Dr. med. M. Weber

Prof. Dr. med. P. Lingor

Prof. Dr. med. M. Weber

Prof. Dr. med. A. Flügel 
Hiermit erkläre ich, die Dissertation mit dem Titel "The effect of dimethyl fumarate on phenotype and function of B lymphocytes in MS patients" eigenständig angefertigt und keine anderen als die von mir angegebenen Quellen und Hilfsmittel verwendet zu haben.

Göttingen, den 11.01.2020 
Die Daten, auf denen die vorliegende Arbeit basiert, wurden teilweise publiziert:

Traub J, Traffehn S, Ochs J, Häusser-Kinzel S, Stephan S, Scannevin R, Brück W, Metz I, Weber MS (2019): Dimethyl fumarate impairs differentiated $\mathrm{B}$ cells and fosters central nervous system integrity in treatment of multiple sclerosis. Brain Pathol 29, 640-657

Posterpräsentation im Rahmen des 34th congress of the European committee for treatment and research in multiple sclerosis (ECTRIMS), Berlin, 09.10.12.10.2018 


\section{Table of contents}

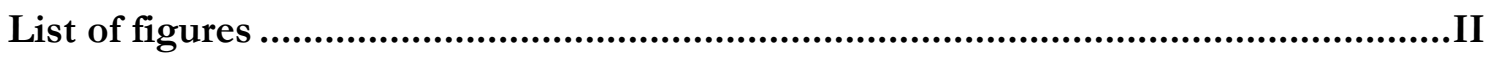

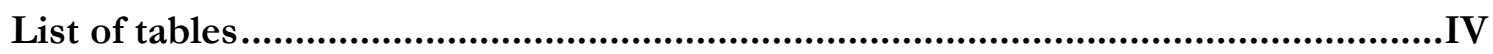

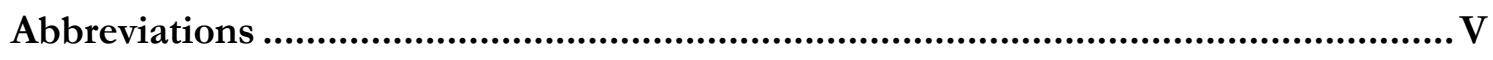

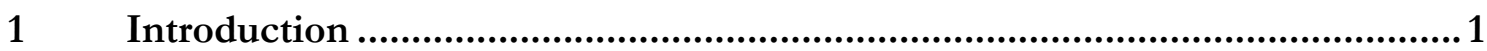

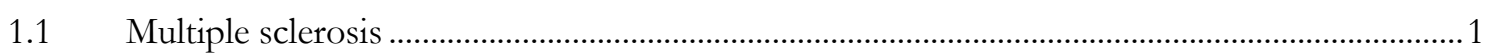

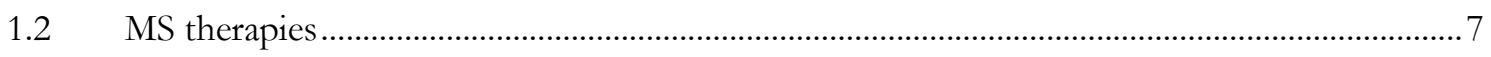

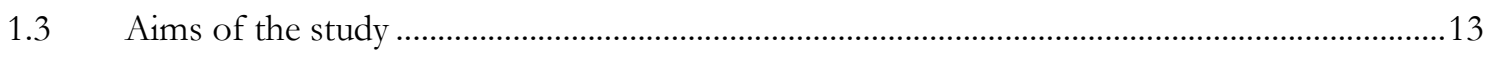

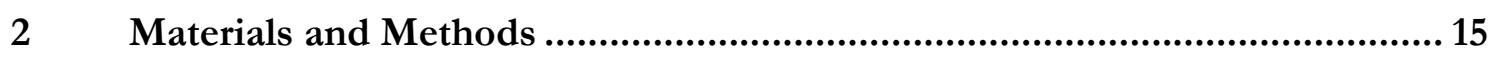

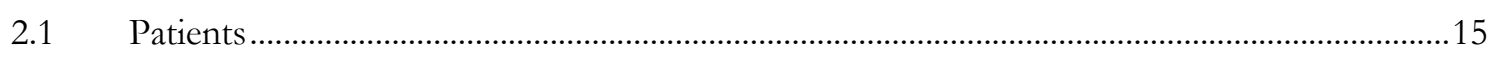

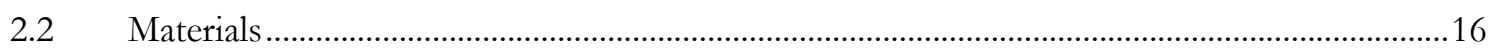

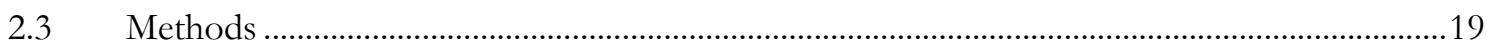

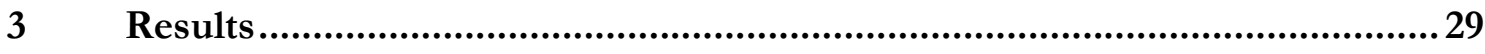

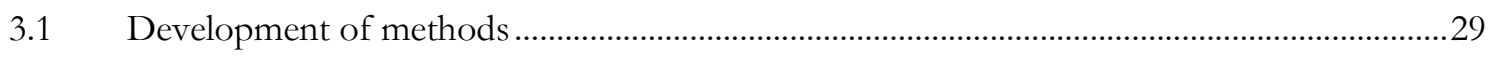

3.2 Cell distribution and survival ................................................................................................. 34

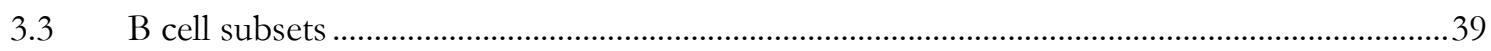

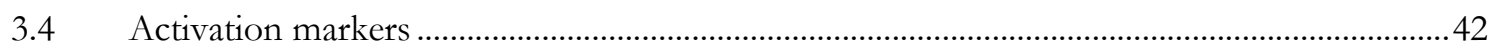

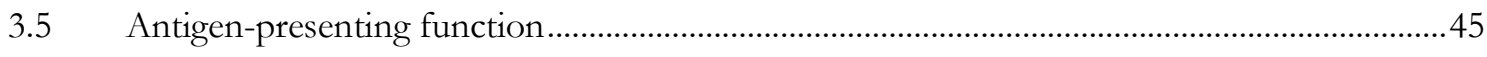

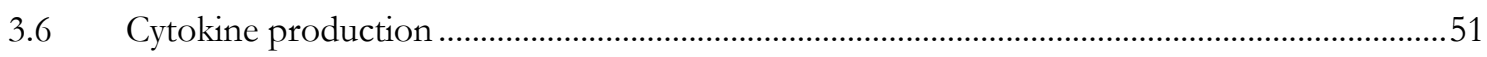

3.7 The influence of treatment duration on DMF-mediated changes in the immune cell

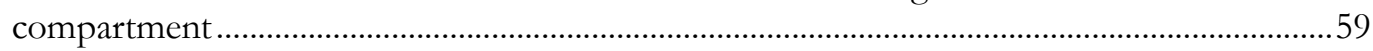

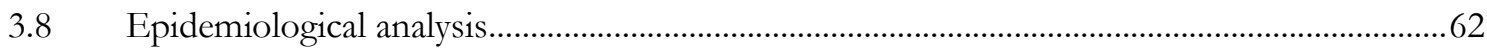

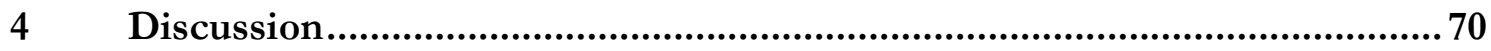

4.1 The effect of dimethyl fumarate on B cells .......................................................................... 70

4.2 The effects of dimethyl fumarate on other leukocytes............................................................... 75

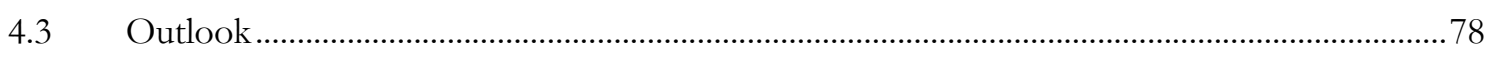

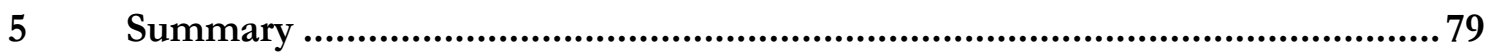

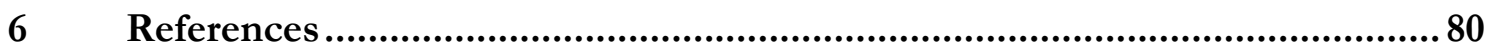




\section{List of figures}

Figure 1: Pre-gating and gating strategy for B cell subsets and surface molecule expression............27

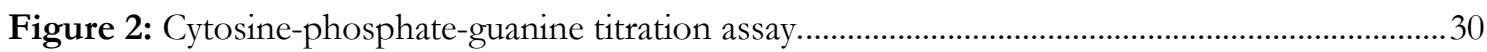

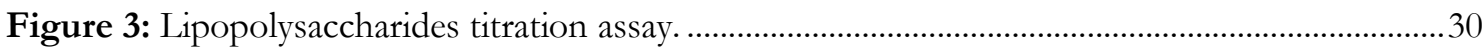

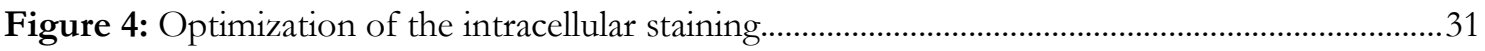

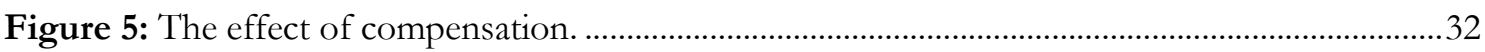

Figure 6: Compensation matrices of the antigen presentation panel (e),..............................................33

Figure 7: Compensation matrices of all five acquisition panels. ........................................................... 34

Figure 8: Dimethyl fumarate decreased the proportion of intact cells (excluded by size)..................35

Figure 9: Upon stimulation, the proportion of living cells was reduced by dimethyl fumarate.........36

Figure 10: Dimethyl fumarate decreased the frequency of living B cells.............................................36

Figure 11: $\mathrm{CD} 14^{+}$myeloid cell frequency increased upon dimethyl fumarate treatment....................37

Figure 12: $\mathrm{CD}^{+}{ }^{+} \mathrm{T}$ cell frequency was not altered by dimethyl fumarate..............................................38

Figure 13: Dimethyl fumarate treatment reduced the frequency of $\mathrm{CD} 8^{+} \mathrm{T}$ cells...............................38

Figure 14: B cell frequency was not altered by dimethyl fumarate.....................................................39

Figure 15: Dimethyl fumarate treatment increased transitional B cell frequency.................................40

Figure 16: Antigen-experienced and memory B cell frequencies were reduced by dimethyl

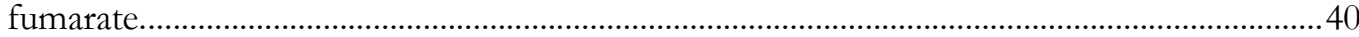

Figure 17: Dimethyl fumarate treatment reduced the frequency of plasmablasts................................ 41

Figure 18: CD25 expression on B cells was reduced by dimethyl fumarate treatment........................42

Figure 19: CD69 was reduced by dimethyl fumarate on cytosine-phosphate-guaninestimulated B cells. …………………………………………………………………………....4

Figure 20: Dimethyl fumarate reduced the expression of CD95 on B cells.......................................43

Figure 21: CD150+ B cells were reduced upon dimethyl fumarate treatment. ....................................4

Figure 22: Dimethyl fumarate did not alter the expression of activation markers on myeloid phagocytes.

Figure 23: Major histocompatibility complex - class II expression on B cells was not altered

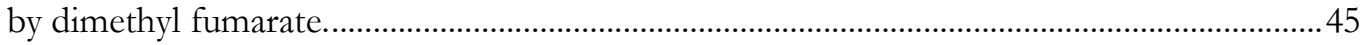

Figure 24: In the longitudinal samples, dimethyl fumarate upregulated major histocompatibility complex - class II on B cells.

Figure 25: Major histocompatibility complex - class II on B cells tended to increase the longer dimethyl fumarate was taken.

Figure 26: Upon stimulation, CD40 expression on B cells was reduced...............................................47

Figure 27: Dimethyl fumarate reduced the expression of CD80 on B cells........................................48

Figure 28: CD86 upregulation upon stimulation was inhibited by dimethyl fumarate.........................48

Figure 29: Dimethyl fumarate did not change the major histocompatibility complex - class II expression on myeloid-derived phagocytes.

Figure 30: The expression of major histocompatibility complex - class II positively correlated with the duration of dimethyl fumarate intake.

Figure 31: Dimethyl fumarate reduced the expression of CD80 on myeloid-derived phagocytes.

Figure 32: Interleukin-6 production of B cells was reduced upon dimethyl fumarate treatment.....51

Figure 33: Tumor necrosis factor alpha production of B cells was decreased upon dimethyl fumarate treatment

Figure 34: Dimethyl fumarate reduced the interleukin-10 production of $B$ cells.

Figure 35: Interleukin-10 / interleukin-6 ratio was increased in B cells upon dimethyl fumarate treatment. 
Figure 36: Dimethyl fumarate treatment elevated the interleukin-10 / tumor necrosis factor alpha ratio in $\mathrm{B}$ cells.

Figure 37: Interleukin-6 production in myeloid-derived phagocytes was reduced by dimethyl fumarate.

Figure 38: Tumor necrosis factor alpha production in $\mathrm{CD}_{14}{ }^{+}$myeloid cells was reduced by dimethyl fumarate.

Figure 39: Dimethyl fumarate did not alter the interleukin-10 production of $\mathrm{CD} 14^{+}$myeloid cells.

Figure 40: Interleukin-10 / interleukin-6 ratio increased in CD14+ myeloid cells upon dimethyl fumarate.

Figure 41: In CD14+ myeloid cells, the interleukin-10 / tumor necrosis factor ratio was not altered by dimethyl fumarate.

Figure 42: The interleukin-6 concentration was not changed in the supernatant. .58

Figure 43: Dimethyl fumarate does not change the concentration of interleukin-10 in the supernatant

Figure 44: Dimethyl fumarate induced an anti-inflammatory shift in the supernatant.

Figure 45: $\mathrm{CD}_{14}{ }^{+}$myeloid cell and $\mathrm{CD}^{+} \mathrm{T}$ cell frequencies as a function of dimethyl fumarate treatment duration.

Figure 46: $\mathrm{CD}^{2} 9^{+}$and $\mathrm{CD} 80^{+} \mathrm{B}$ cell frequencies as a function of dimethyl fumarate treatment duration.

Figure 47: Correlation between cell frequencies and patient-related data......................................64

Figure 48: Correlation between B cell subsets and patient-related data..........................................65

Figure 49: Correlation between cell B cells subsets and patient-related data...................................66

Figure 50: Correlation between antigen-presenting function relevant markers and patientrelated data.

Figure 51: Correlation between B cell cytokine production and patient-related data.

Figure 52: Correlation between myeloid-derived phagocyte cytokine production and patientrelated data. 


\section{List of tables}

Table 1: McDonald criteria (Thompson et al. 2018) ........................................................................ 4

Table 2: Demographic and disease characteristics of the patients.....................................................15

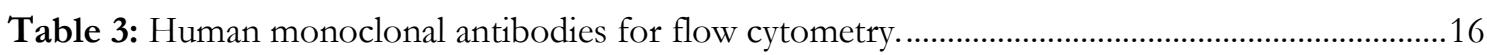

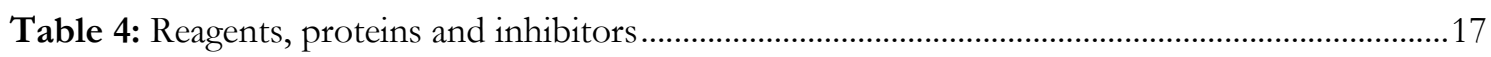

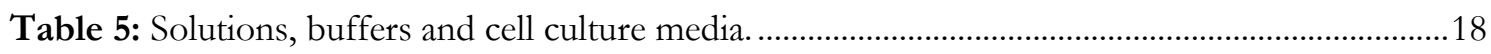

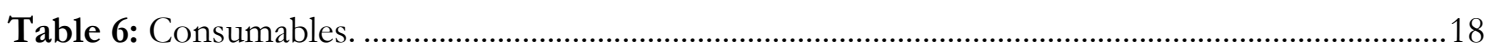

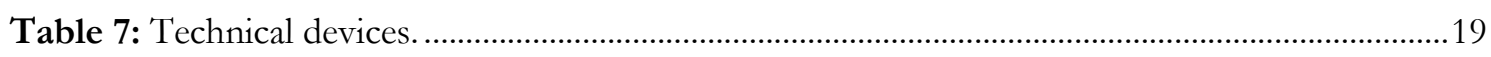

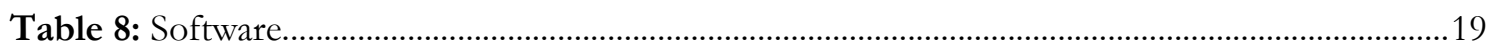

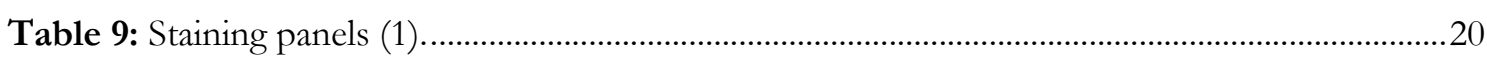

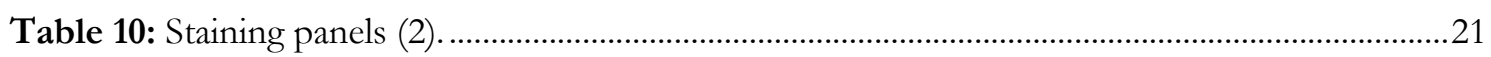

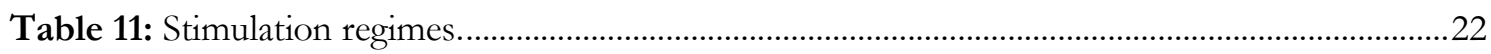

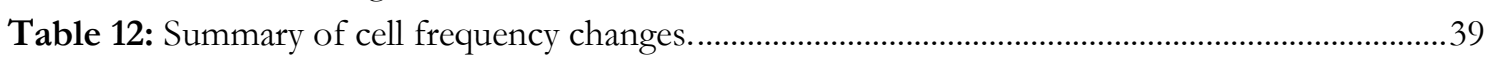

Table 13: Summary of B cell subset changes. ......................................................................................41

Table 14: Summary of dimethyl fumarate-induced changes of B cell activation markers.................44

Table 15: Dimethyl fumarate-induced changes of molecules involved in antigen presentation

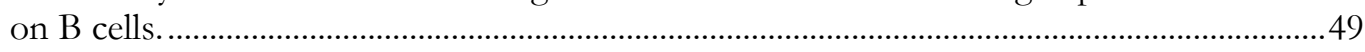

Table 16: Dimethyl fumarate-induced changes of the cytokine production of $B$ cells......................54

Table 17: Dimethyl fumarate-induced changes of the cytokine production of CD14+ myeloid

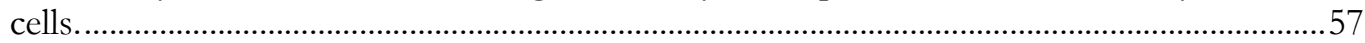

Table 18: Correlation of cell frequencies with duration of dimethyl fumarate medication...............59

Table 19: Correlation between B cell subset frequency and the duration of dimethyl fumarate intake.

Table 20: Correlation between cell surface markers and the duration of dimethyl fumarate intake. 


\section{Abbreviations}

\begin{tabular}{|c|c|}
\hline APC & antigen-presenting cells \\
\hline BAFF & B cell activation factor \\
\hline BBB & blood-brain barrier \\
\hline BSA & bovine serum albumin \\
\hline CD & cluster of differentiation \\
\hline CIS & clinically isolated syndrome \\
\hline CNS & central nervous system \\
\hline CpG & cytosine-phosphate-guanine \\
\hline CSF & cerebrospinal fluid \\
\hline CTLA-4 & cytotoxic T lymphocyte-associated protein \\
\hline dil. fac. & dilution factor \\
\hline DIS & dissemination in space \\
\hline DIT & dissemination in time \\
\hline DMEM & Dulbecco's Modified Eagle's Medium \\
\hline DMF & dimethyl fumarate \\
\hline DMSO & dimethyl sulfoxide \\
\hline EAE & experimental autoimmune encephalitis \\
\hline EDSS & expanded disability status scale \\
\hline EDTA & ethylene diamine tetraacedic acid disodium salt dihydrate \\
\hline ELISA & enzyme-linked immunosorbent assay \\
\hline EMA & European Medicines Agency \\
\hline FACS & fluorescence-activated cell sorting \\
\hline FCS & fetal calf serum \\
\hline FDA & US Food and Drug Administration \\
\hline FSC & forward scatter \\
\hline HCAR2 & hydroxyl-carboxylic acid receptor 2 \\
\hline IFN- $\beta$ & interferon-beta \\
\hline IFN- $\gamma$ & interferon-gamma \\
\hline IgG & immunoglobulin $\mathrm{G}$ \\
\hline IL & interleukin \\
\hline LINGO-1 & $\begin{array}{l}\text { leucine rich repeat and immunoglobulin-like domain containing } \\
\text { protein } 1\end{array}$ \\
\hline LPS & lipopolysaccharides \\
\hline MAPK & mitogen-activated protein kinase \\
\hline MFI & mean fluorescence intensity \\
\hline MHC-II & major histocompatibility complex - class II \\
\hline MMF & monomethyl fumarate \\
\hline MOG & myelin oligodendrocytic glycoprotein \\
\hline MRI & magnetic resonance imaging \\
\hline MS & multiple sclerosis \\
\hline
\end{tabular}




\begin{tabular}{ll} 
Nrf2 & nuclear factor erythroid 2-related factor \\
NF- $\boldsymbol{x}$ B & nuclear factor 'kappa-light-chain-enhancer' of activated B cells \\
OCB & oligoclonal band \\
p.o. & per os \\
PAMP & pathogen-associated molecular pattern \\
PBMC & peripheral blood mononuclear cells \\
PBS & phosphate-buffered salt solution \\
PMA & phorbol 12-myristate 13-acetate \\
PML & progressive multifocal leukoencephalopathy \\
PPMS & primary progressive multiple sclerosis \\
PRMS & progressive-relapsing multiple sclerosis \\
RIS & radiologically isolated syndrome \\
rpm & revolutions per minute \\
RPMI-1640 & Roswell Park Memorial Institute-1640 \\
RRMS & relapsing-remitting multiple sclerosis \\
s.c. & subcutaneous \\
SPMS & secondary progressive multiple sclerosis \\
Th & T helper \\
TLR & toll-like receptor \\
TMB & tetramethylbenzidine \\
TNF & tumor necrosis factor \\
TRAF2 & TNF receptor-associated factor 2 \\
\hline
\end{tabular}




\section{Introduction}

\subsection{Multiple sclerosis}

Millions of patients worldwide are affected by multiple sclerosis (MS), a chronic inflammatory demyelinating disease of the central nervous system (CNS) (Nakahara et al. 2012). The disease was first described in the year 1838 by Robert Carswell, who illustrated lesions of the brain and the spinal cord. It is characterized by the pathological hallmarks inflammation, demyelination, axonal loss and gliosis (Bruck and Stadelmann 2003). The exact cause remains unknown while a combination of genetic, environmental and infectious factors is being discussed (Compston and Coles 2008). There is no known cure for MS; treatment strategies try to prevent or ameliorate relapses and to slow down chronic progression.

\subsubsection{Epidemiology, etiology and impact}

In 2018, about 2.3 million people worldwide were suffering from MS. In Germany, the prevalence is estimated to 149 cases per 100,000 inhabitants (Hein and Hopfenmüller 2000). Several studies from Tasmania, Spain and Norway have revealed a strong increase in prevalence and incidence of MS in the last decades (Grytten et al. 2015; Izquierdo et al. 2015; Simpson et al. 2011). It is twice as common in women than in men and usually begins between the age of 20 and 50 (Milo and Kahana 2010). The development of modern diagnostic tools such as magnetic resonance imaging (MRI) and a higher life expectancy of MS patients might be the cause for the rising prevalence.

Although the prevalence and incidence vary strongly among different populations and regions, a strong correlation between latitude and the incidence of MS can be observed (Simpson et al. 2011). Interestingly, this effect has been seen in genetically homogenous populations as well as in people that migrated to a country with a higher latitude. Therefore, many investigations on the effect of vitamin D in MS are being performed (PierrotDeseilligny and Souberbielle 2013).

The exact cause of MS is still unknown. Many genes that increase the risk to develop MS are associated with immunologically relevant proteins like human leukocyte antigen - D related, interleukin (IL)-10, C-C chemokine receptor type 5 and interferon-gamma (IFN- $\gamma$ ). Environmental risk factors include infections with Ebstein-Barr virus, human herpesvirus 6 and herpes simplex virus 6 , low vitamin D levels as well as geographic factors, such as increasing distance to the equator (Sospedra and Martin 2005). It was also shown that a high salt consumption and a "western diet" increase the risk for MS (Kleinewietfeld et al. 2013; Manzel et al. 2014). 
MS has a low mortality, but is the most common reason for disability in young adults (Raffel et al. 2017). Employment rates of MS patients drastically decrease with disease progression (Dorstyn et al. 2017). The same effect can be seen when quality of life is assessed (Orme et al. 2007). The healthcare costs for one patient are approximately $€ 31.000$ per year (Fernández et al. 2017).

\subsubsection{Symptoms and forms}

Depending on the affected CNS region, almost any neurological symptom or sign can be observed in MS patients. Very common are visual symptoms, including nystagmus, optic neuritis and diplopia. Anxiety, fatigue, cognitive impairment, depression and instable mood represent central symptoms. Speech may be affected through dysarthria and many patients show symptoms of dysphagia. Musculoskeletal problems include spasms, ataxia and weakness, whereas sensation is affected by pain, hypoesthiasis and paraesthiasis. MS symptoms can furthermore affect the gastrointestinal tract: Incontinence, diarrhea or constipation are seen in many patients. In addition, urinary problems such as incontinence or retention can be observed (Milo and Kahana 2010). Due to the variability and complexity of the clinical appearance, the disease activity is quantified with the expanded disability status scale (EDSS) score, reaching from zero (no clinical symptoms) to ten (death), focusing mainly on motoric symptoms (Kurtzke 1983).

There are different patterns of progression in MS. In 1996, the US National Multiple Sclerosis society distinguished relapsing-remitting MS (RRMS), secondary progressive MS (SPMS), primary progressive MS (PPMS) and progressive-relapsing MS (PRMS) based on the clinical course at the onset of the disease (Lublin et al. 2014).

Because of a deeper understanding of MS in the following years, PRMS was removed in 2014 and the two new phenotypes clinically isolated syndrome (CIS) and radiologically isolated syndrome (RIS) were added (Lublin et al. 2014).

RIS represents the most frequent form of asymptomatic MS, where MRI suggests MS lesions, but the patients are symptom-free (Granberg et al. 2013). In most cases though, MS begins with a CIS, a condition where symptoms occur, but patients do not fulfil all MS criteria. Subsequently, 70\% of those patients develop an RRMS phenotype (Miller et al. 2005). This is the most common subset affecting almost $90 \%$ of all MS patients (Compston and Coles 2002). It is characterized by unpredictable relapses, defined as an acute worsening of the disease within days or weeks, followed by complete or partial recovery. Between the relapses, the clinical course is usually stable. "Benign MS" describes the condition where symptoms always resolve after a relapse (Pittock and Rodriguez 2008).

Almost $70 \%$ of the RRMS cases however convert into SPMS over time when recovery from relapse becomes incomplete and disability accumulates (Weinshenker 1998). This progress usually takes about 20 years (Rovaris et al. 2006). Rarely, there might be occasional relapses with minor remissions also in the secondary progressive phase (Lublin et al. 1996). 
A small proportion of MS patients has no remission at all after the initial symptoms. This entity is called PPMS (Thompson et al. 1997). The disability continuously worsens while plateaus or minor improvements might occur. At the onset of disease, patients with PPMS are about 10 years older than RRMS patients (Miller and Leary 2007). Whereas in RRMS, symptoms develop rapidly within days, this process takes months or years in PPMS. Spastic and ataxic paresis occur as a result of a progressive myelopathy (Ontaneda and Fox 2015).

Occasionally, atypical forms of MS occur, including Marburg's acute MS, Balò's concentric sclerosis and Schilder's diffuse sclerosis, which are very rare and potentially considered as distinct diseases (Stadelmann and Brück 2004).

\subsubsection{Diagnosis}

As described above, the clinical presentation of MS is very heterogeneous. Hence, the diagnosis is based on clinical symptoms combined with radiological imaging and laboratory data.

To the present day, MS is commonly diagnosed according to the McDonald criteria (Table 1), which were introduced in 2001 and reviewed three times in the following years (McDonald et al. 2001; Polman et al. 2011; Polman et al. 2005; Thompson et al. 2018). Accordingly, the diagnosis is based on three features: clinical symptoms, disease evidence assessed by MRI and cerebrospinal fluid diagnostics.

To improve the McDonald criteria of 2010, the magnetic resonance imaging in multiple sclerosis (MAGNIMS), a European collaborative research network, published new MRI recommendations in 2016. In those, MRI findings accurately prove dissemination in space (DIS) and dissemination in time (DIT), excluding diseases with symptoms similar to MS (Filippi et al. 2016).

Laboratory analysis of cerebrospinal fluid (CSF) may detect elevated, intrathecally produced immunoglobulin (Ig)G levels, which are seen in $75 \%$ of all MS patients (Link and Huang 2006). To discover deficits in clinically silent cases, evoked potential testing, for example for sight, vision or motor function, can be used, as MRI hardly detects very small lesions. 
Table 1: McDonald criteria (Thompson et al. 2018)

\begin{tabular}{|c|c|c|}
\hline $\begin{array}{l}\text { Clinical } \\
\text { attacks }\end{array}$ & Objective clinical lesions & Additional criteria to make diagnosis \\
\hline 2 or more & 2 or more & None; clinical evidence will suffice \\
\hline 2 or more & $\begin{array}{l}1 \text { (as well as clear-cut historical } \\
\text { evidence of a previous attack } \\
\text { involving a lesion in a distinct } \\
\text { anatomical location) }\end{array}$ & None; clinical evidence will suffice \\
\hline 2 or more & 1 & $\begin{array}{l}\text { Dissemination in space (DIS), demonstrated by } \\
\text { MRI or further clinical attack involving } \\
\text { different site. }\end{array}$ \\
\hline 1 & 2 or more & $\begin{array}{l}\text { Dissemination in time (DIT) demonstrated by } \\
\text { an additional clinical attack or by magnetic } \\
\text { resonance imaging (MRI) OR demonstration of } \\
\text { cerebrospinal fluid (CSF)-specific oligoclonal } \\
\text { bands }\end{array}$ \\
\hline 1 & 1 & $\begin{array}{l}\text { DIS and DIT (or CSF-specific oligoclonal } \\
\text { bands) }\end{array}$ \\
\hline $\begin{array}{l}\text { 0 } \\
\text { (progression } \\
\text { from onset) }\end{array}$ & 0 & $\begin{array}{c}\text { One year of disability progression } \\
\text { (retrospectively or prospectively determined) } \\
\text { independent of clinical relapse PLUS two of the } \\
\text { following criteria: } \\
\text { - One or more T2-hyperintense lesions characteristic } \\
\text { of multiple sclerosis in one or more of the following } \\
\text { brain regions: periventricular, cortical or } \\
\text { juxtacortical, or infratentorial } \\
\text { - Two or more T2-hyperintense lesions in the spinal } \\
\text { cord } \\
\text { - Presence of CSF-specific oligoclonal bands }\end{array}$ \\
\hline
\end{tabular}

\subsubsection{Immunopathogenesis and pathology}

Most theories on the pathophysiology of MS can be divided into two major models: The inside-out or the outside-in theory. According to the outside-in theory, the disease is initiated by the peripheral immune system, where pathogenic, autoreactive $\mathrm{T}$ cells are activated by viral, environmental and/or genetic stimuli mentioned above (Hemmer et al. 2015). Despite intensive investigations however, no specific antigen evoking such an autoimmune response could be identified so far (Banki et al. 1994; Wucherpfennig et al. 1997). 
Regarding disease pathogenesis, activated autoreactive cluster of differentiation (CD) $4^{+} \mathrm{T}$ cells are thought to upregulate adhesion molecules and bind certain receptors of the endothelium allowing them to cross the blood-brain barrier (BBB). Inside the CNS, they are reactivated by local antigen-presenting cells (APC), on which they recognize their specific antigen. In the process of reactivation, they differentiate into $T$ helper $\left(T_{h}\right) 1$ and $T_{h} 17$ cells. By secreting pro-inflammatory cytokines such as IFN- $\gamma$ and IL-17, they are assumed to trigger an increased production of chemokines. In turn, this causes the endothelial cells to upregulate the expression of adhesion molecules and attracts other cellular components of the immune system like monocytes, dendritic cells, T cells, B cells and plasma cells to invade CNS tissue as the BBB has become penetrable (Bartholomäus et al. 2009). It is assumed that these infiltrating cells lead to a general inflammatory condition within the CNS. Being stimulated by tumor necrosis factor (TNF) and IFN- $\gamma$, APC are thought to upregulate distinct surface molecules and hereby perpetuate $\mathrm{T}$ cell activation within the CNS. As a consequence, macrophages and microglia release reactive oxygen and nitrogen species that can injure oligodendrocytes and myelin sheaths (Fischer et al. 2012). Further damage is assumed to be caused by cytotoxic $\mathrm{CD}^{+} \mathrm{T}$ cells that attack glial cells directly. The invading peripheral immune cells together then form the typical inflammatory demyelinating lesions that can be observed in MRI and histology.

Early-active lesions are characterized by demyelinating areas that contain varying numbers of macrophages filled with myelin degradation products, B cells, plasma cells, and perivascular and parenchymal $\mathrm{T}$ cell infiltrates. According to their cellular composition, they can be further subdivided into four different patterns (Lucchinetti et al. 2000). Incomplete demyelination, acute axonal damage but also regeneration are typical signs for acute lesions (Kuhlmann et al. 2002). Because of a disrupted BBB, they correlate with the presence of gadolinium-enhancing lesions in the MRI.

In contrast, progressive forms of MS show a lower number of newly-forming inflammatory lesions. Grey and white matter atrophy is accompanied by gradual expansion of existing lesions where macrophages with phagocyted myelin can be seen only at the lesion border and where just few lymphocytes are present within the lesion (Lassmann 2010). Moreover, normal-appearing white matter shows signs of diffuse abnormal inflammation and oligodendrocyte precursor cells are more prominent than mature oligodendrocytes (Nylander and Hafler 2012).

Supporting the inside-out theory, some authors however claimed that inflammation begins within the CNS, as early lesions show high microglial activation with only few leukocytes, assuming that these pro-inflammatory microglia are causing the BBB breakdown (Barnett and Prineas 2004). Further, it has been discussed that the starting point of MS are aggregates of microglia that represent an initial brain damage. Subsequently, CNS-specific antigens can be drained from the brain tissue through lymphatic vessels into cervical lymph nodes and activate immune cells in the periphery (Stys et al. 2012; Kipnis 2016). 


\subsubsection{The role of $B$ cells}

The most compelling evidence for the involvement of B cells in MS was the astonishing success of B cell-depleting therapies using monoclonal antibodies against CD20, such as rituximab and ocrelizumab (Hauser et al. 2008; Hauser et al. 2017). The efficiency of those anti-CD20 antibody therapies is thought to be related to the loss of B cell cytokine-producing and antigen-presenting functions, whereas pathogenic autoantibodies seem to play a minor role (Li et al. 2015).

Thus, the classical focus on $\mathrm{T}$ cells as the only relevant key effector cell type in the pathophysiology of MS has been questioned in the past years (Franciotta et al. 2008). Mature naive B cells are very efficient APC and interact with $\mathrm{CD}^{+}{ }^{+} \mathrm{T}_{\mathrm{h}}$ cells to become fully activated. Therefore, B cells encounter antigen specifically via their B cell receptor, process it to linearized peptides and present it via the major histocompatibility complex - class II (MHCII) to $\mathrm{CD}^{+}{ }^{+} \mathrm{T}$ cells. Subsequently, responding $\mathrm{T}$ cells are activated and differentiate into distinct effector $\mathrm{T}$ cells depending on the received stimuli. In turn, the presenting $\mathrm{B}$ cell can differentiate into either memory B cells or antibody-producing plasma cells (Franciotta et al. 2008). Furthermore, B cells are a relevant source of different cytokines. On the one hand, they are able to produce the pro-inflammatory cytokines IL-6, TNF and IFN- $\gamma$ that promote the immunological answer to pathogens, in autoimmune diseases however also to the body's own structures (Krumbholz and Meinl 2014). On the other hand, by producing IL-10 and IL-35, they can also downregulate inflammation by inducing anti-inflammatory processes (Fillatreau et al. 2002).

The first evidence for the pathogenic involvement of $\mathrm{B}$ cells were oligoclonal bands (OCB) discovered in the CSF of MS patients more than 50 years ago (Lowenthal et al. 1960). Recent data indicated that those OCB originate from plasma cell clones within the brain parenchyma and the CSF (Obermeier et al. 2011). More precisely, they seem to be located perivascular in both early and chronic MS lesions, where they are assumed to produce immunoglobulin G (IgG) antibodies (Henderson et al. 2009). It has been demonstrated that these antibodies can be directed against various antigens including multiple viruses, nuclear components and brain antigens, but also so-called "nonsense antigens", meaning that they are not directed against a known structure. Moreover, B cells can be found in the meninges of MS patients where they form B cell-rich structures that resemble tertiary B cell follicles (Howell et al. 2011).

These findings mainly focused on the relevance of antibodies produced by plasma cells. In the last years though, it has been shown that other cellular B cell properties may be more crucial for the development of MS, since the clinical effect of anti-CD20 therapies was not on account of reduced antibody level (Hauser et al. 2008). In this context, one potentially important function of B cells may be their capacity to act as APC: Costimulatory molecules, such as CD80 and CD86, that are essential for the interaction between B and T cells, are upregulated in MS patients (Fraussen et al. 2014). These findings indicate that pathogenic B cells may be involved in a CNS-directed autoimmune reaction. In addition, the cytokine 
production of B cells in MS patients is altered. They show a higher production of the inflammatory cytokines IL-6 and TNF when compared to healthy controls (Barr et al. 2012). Investigating the myeloid cell-secreted B cell activation factor (BAFF), an important survival stimulus especially for auto-aggressive B cells subtypes, it was shown that the concentration of BAFF is elevated in MS lesions and on B lymphocytes in the blood (Krumbholz et al. 2005; Montes Diaz et al. 2018). In contrast, the number of regulatory B cells, that can produce the anti-inflammatory cytokines IL-10 and IL-35, were much lower in the peripheral blood of MS patients compared to healthy controls (Knippenberg et al. 2011). This indicates that the regulatory functions of $\mathrm{B}$ cells, such as suppression of $\mathrm{T}$ cell activation and induction of regulatory T cells may be severely limited in MS.

It must be noted that compared to $\mathrm{T}$ cells and macrophages, $\mathrm{B}$ cells and plasma cells are rather rare in MS lesions. However, "pattern II" lesions, that show immunoglobulin and complement deposition and require plasma cell function, are the most frequent lesion subtype found in MS patients (Lassmann et al. 2007). As B cells are mostly found in early and active lesions, it is assumed that their relevance is higher in those early lesions (Michel et al. 2015).

These findings and descriptions emphasize that B cells are important in the pathophysiology of MS. Thereby, their altered phenotype and enhanced pro-inflammatory properties in MS patients indicate that they are actively involved in promoting inflammation.

\subsection{MS therapies}

Down to the present day, there is no known cure for MS. Most approved drugs harbor immune-modulating properties and aim to obviate rather than repair tissue injury. In doing so, they prevent new attacks and slow down disability progression. Therapeutic options depend on the progress of the disease and require an individual risk-benefit evaluation.

\subsubsection{Treatment of acute attacks/relapses}

A relapse is defined as an acute worsening of symptoms within days or weeks and symptoms must last at least 24 hours. During those attacks, an intravenous application of high-dosed glucocorticoids such as methylprednisolone is the standard therapy. The common dosage is $1000 \mathrm{mg} / \mathrm{d}$ for 3-5 days (Compston and Coles 2008). Oral administration is also possible and seems to have a similar safety profile with comparable efficacy (Burton et al. 2012). Long-term recovery however is not improved. If this treatment fails or is not tolerated, immunoadsorption or plasma exchange can be performed (Faissner et al. 2016).

\subsubsection{Disease-modifying therapies}

As of 2019, there are currently twelve drugs approved for RRMS. Generally, a combination of different MS medications or dose escalation are not recommended. According to the 
current European guidelines, interferon-beta (IFN- $\beta$; subcutan (s.c.) /intramuscular), glatiramer acetate (GA; s.c.), teriflunomide (per os (p.o.)) and dimethyl fumarate (DMF; p.o.) are used as basic therapy in patients with low disease activity (Montalban et al. 2018).

These drugs are generally well tolerated and show a relatively high safety profile (Cross and Naismith 2014). All four drugs reduce relapse rates and decrease the rate of new MRI lesions and disability progression (Bar-Or et al. 2014; Paty and Li 1993; Mikol et al. 2008; Gold et al. 2012).

If the basic therapy does not suffice and the disease progresses, another basic therapy or a so-called escalation therapy should be chosen. Consequently, patients with a high disease activity can be treated with fingolimod (p.o.), natalizumab (intravenous (i.v.)), alemtuzumab (i.v.) or ocrelizumab (i.v.).

These drugs are therapeutically more efficient than the basic therapies, but not as safe and well tolerated. Several phase III studies confirmed their huge effect on disability progression, new MRI lesions and relapse rates.

Alternative escalation therapy drugs that showed higher efficacy than interferons, include daclizumab (i.v.), which was withdrawn from the marked in 2018, and mitoxanthrone (i.v.) (Hauser et al. 2017; Martinelli Boneschi et al. 2005; Wynn et al. 2010).

For PPMS, only few therapeutic options are available. Most tested disease-modifying therapies did not show any beneficial effects (Ontaneda and Fox 2015). Only the $\mathrm{B}$ cell-depleting monoclonal antibody rituximab has been widely used off-label for many years in PPMS patients, until its further humanized successor ocrelizumab has been approved by the US Food and Drug administration (FDA) in March 2017 and by the European Medicines Agency (EMA) in February 2018 (McGinley et al. 2017).

For the treatment of SPMS only mitoxanthrone has been approved (Scott and Figgitt 2004). While the number of inflammatory infiltrates was reduced, disease progression could not be stopped by this treatment (Ontaneda and Fox 2015).

\subsubsection{Treatment to repair tissue}

The limited success of immunomodulation in progressive forms of MS shows that the development of neuroprotective and repairing therapies is highly desirable. At the moment, there is no approved neuroprotective or repairing drug on the market, but there is an ongoing trial called MS-SMART, that evaluates the effect of three neuroprotective agents (amiloride, riluzole and fluoxetine) in patients with SPMS (Connick et al. 2018).

Another drug that shows promising results in remyelination is a leucine-rich repeat and immunoglobulin-like domain-containing protein 1 (LINGO-1) antagonist. LINGO-1 is a Nogo receptor-interacting protein that appears to be a negative regulator of oligodendrocyte differentiation and myelination (Mi et al. 2007). 


\subsubsection{Treatment of symptoms}

Some symptoms like spasticity, depression, fatigue and unstable bladder can be improved by neuro-rehabilitation or medication, while the disease progression is not affected (Kesselring and Beer 2005). The best way to reduce neurological symptoms is a multidisciplinary approach that increases the patients' activity and participation. Physical exercise, psychological therapy and behavioral cognitive approaches can lead to a higher life quality of MS patients (Khan et al. 2007).

\subsubsection{Dimethyl fumarate}

Being the methyl ester of the fumaric acid, DMF itself is a small molecule with the chemical formula $\mathrm{C}_{6} \mathrm{H}_{8} \mathrm{O}_{4}$. As lipophilic oral agent named BG-12 (tradename Tecfidera), DMF has been approved for the treatment of RRMS in 2014.

Fumarates were originally developed as radio sensitizers to make tumor cells more vulnerable to radiotherapy (Held et al. 1988). Already in the year 1957, the biochemist Schwekendiek successfully tested DMF against psoriasis in a self-experiment (Schweckendieck 1959). Based on successful clinical trials, DMF was officially permitted for the treatment of psoriasis in 1994 as the active substance of the oral drug Fumaderm (Altmeyer et al. 1994). DMF also showed a beneficial effect in other chronic autoimmune diseases, such as granuloma anulare, necrobiosis lipoidica, Crohn's disease and sarcoidosis (Meissner et al. 2012).

In the beginning of the $21^{\text {st }}$ century, German physicians observed that psoriasis patients taking DMF that suffered from MS at the same time, had reduced relapse rates and less disability progression (Reich et al. 2009). Based on these observations and the fact that both psoriasis and MS are considered T cell-driven diseases, clinical investigations on the effect of DMF in MS patients were initiated (Meissner et al. 2012; Reich et al. 2009).

\subsubsection{Clinical trials}

Being considered effective in MS, Fumaderm, a composite of different ethyl hydrogen fumarate-salts and dimethyl fumarate was tested in an exploratory, prospective, open-label study in patients with RRMS in 2006. The trial showed that Fumaderm modulates T cellmediated cytokine production and significantly reduces frequency and number of new gadolinium-enhancing MRI lesions (Schimrigk et al. 2006). These promising results lead to the development of an oral formulation of dimethyl fumarate, named BG-12, which was tested in a Phase IIb study involving 257 patients with RRMS. Similar to Fumaderm, BG-12 reduced the relapse rates significantly by $32 \%$ and new gadolinium-enhancing lesions by $69 \%$ when patients received $240 \mathrm{mg}$ three times a day. Furthermore, fewer new or enlarging T2hyperintense and new T1-hypointense lesions were observed upon treatment (Kappos et al. 2008). 
Based on these findings, two randomized, controlled phase III studies were conducted. The DEFINE study included 1237 patients with RRMS and could confirm that BG-12 significantly reduces the proportion of patients with a relapse, the annualized relapse rate, the rate of disability progression and the number of lesions on MRI when compared to placebo. Evaluating the dosage, there was no big difference in the patients' outcome when comparing twice or three times daily BG-12 intake (Gold et al. 2012). The other performed phase III study was called CONFIRM and tested the effect of BG-12 compared to placebo and glatiramer acetate in 1430 patients. It demonstrated similar effects to the DEFINE study: BG-12 (taken twice of thrice daily) significantly reduced relapse rates and improved neuroradiologic outcomes relative to placebo. When compared to GA, BG-12 was superior in the annualized relapse rate and new or enlarging MRI lesions (Fox et al. 2012).

Here upon, BG-12 was marketed under the name Tecfidera and was approved for the treatment of RRMS by the FDA in 2013 and by the EMA in 2014. Being administrated orally, the recommended dosage is $120 \mathrm{mg}$ twice a day for the first week of treatment and $240 \mathrm{mg}$ thereafter. Age, gender and weight do not require a dosage adjustment (Montalban et al. 2018).

\subsubsection{Side effects and safety concerns}

Generally, DMF is considered an immunomodulatory drug with a reasonable safety profile. In the course of the aforementioned studies, it was found that adverse events upon DMF treatment include abdominal pain, flushing, and hot flush. Dose-related adverse events were headache, fatigue and hot flashes. In some cases, increased liver enzymes and microalbuminuria were observed (Kappos et al. 2008).

Although DMF is considered rather immunomodulatory than immunosuppressive, there is a risk of developing a critically low lymphocyte cell count. It was shown that lymphocyte monitoring before and during DMF treatment helps identifying patients at risk for developing severe, prolonged lymphopenia (Fox et al. 2016).

In some rare cases, low lymphocyte counts due to DMF intake lead to the development of progressive multifocal leukoencephalopathy (PML). At the present day, five cases of PML in MS patients treated with DMF have been reported, with one patient not surviving this complication. In one of the mentioned cases, PML occurred in a patient with normal lymphocyte counts (Nieuwkamp et al. 2015; Rosenkranz et al. 2015; van Kester et al. 2015; van Oosten et al. 2013). These safety concerns suggest that down to the present day, the exact mechanism of action of DMF and especially its effects on the immune system are not yet fully understood.

\subsubsection{Mode of action}

Esterases in the human gut are thought to hydrolyze DMF quickly, effective and pre-systemic into monomethyl fumarate (MMF). This molecule is more stable in blood than DMF and 
assumed to be its active metabolite (Schmidt et al. 2007). Consequently, most biological effects of DMF are supposed to be MMF-mediated. For DMF/MMF, two major modes of action are proposed: Antioxidative/neuroprotective and immunomodulatory effects.

To investigate these effects in a more detailed manner, several in vivo and vitro studies have been performed. Murine MS models include the experimental autoimmune encephalitis (EAE), where the injection of myelin oligodendrocyte glycoprotein (MOG) peptides induces neuroinflammation and the toxic cuprizone model leading to oligodendrocyte death and a subsequent reversible demyelination (Torkildsen et al. 2008). Those models confirmed the clinical benefits observed in patients: when mice were treated with DMF, both in a preventive and in a therapeutic setting, clinical symptoms improved (Traub et al. 2019; Chen et al. 2014; Linker et al. 2011). In vitro studies helped elucidating direct effects of MMF/DMF on immune cells or CNS-derived cells, respectively.

\subsubsection{Antioxidative effects}

The assumption that DMF has anti-oxidative effects is based on several data derived from EAE, in vitro experiments and studies of human biopsies: In vivo experiments in mice showed that DMF significantly promoted preservation of myelin and axons and diminished astrogliosis in the MOG peptide (35-55)-induced EAE. When analyzed histologically, DMF-treated mice had a higher number of surviving neurons compared to controls. It is suggested that this beneficial effect is mediated by an upregulation of antioxidant response pathways (Thiessen et al. 2010). In this regard, nuclear factor erythroid 2-related factor (Nrf2), a transcription factor that promotes the production of anti-oxidative enzymes is assumed to be the key target of DMF. One of the enzymes regulated by Nrf2, the nicotinamide adenine dinucleotide phosphate quinolone oxidoreductase-1, was increased in the liver and cerebellum of MOG peptide immunized rats (Linker et al. 2011). Further upregulated enzymes are glutathione-reductase 1, glutathione-S-transferase, uridine diphosphate-glucuronosyltransferase, hemoxygenase and multi drug resistance proteins. Another study could reveal that the concentration of Nrf2 is increased in glial cells and neurons of DMF-treated animals compared to the control group (Scannevin et al. 2012). In human biopsies, DMF treatment led to a translocation of Nrf2 into the nuclei, predominantly in astrocytes (Metz et al. 2015). Further prove of the Nrf2-dependent effect derived from the observation that Nrf2 knockout mice had no clinical benefit of DMF treatment (Linker et al. 2011). The same effect was seen in mice that did not express the hydroxyl-carboxylic acid receptor 2 (HCAR2) indicating that this molecule may also be relevant for the beneficial effects of DMF (Chen et al. 2014). In the cuprizone mouse model, feeding of this agent leads to toxic demyelination, which is thought to be independent of the peripheral immune system. In this model, DMF could slightly accelerate re-myelination and reduce acute axonal damage (Moharregh-Khiabani et al. 2010; Traub et al. 2019). Furthermore, it has been reported that in mice, DMF protects neural stem/progenitor cells from oxidative damage through the 
aforementioned Nrf2 - mitogen-activated protein kinase (MAPK) pathway by upregulating anti-oxidative enzymes (Wang et al. 2015).

In vitro studies confirmed the beneficial effect of DMF on the survival of neurons and their axons, which was associated with a reduced astroglial activation (Reick et al. 2014). Moreover, Nrf2 was shown to be upregulated in microglia, endothelial cells, astrocytes, neurons and oligodendrocytes when they were exposed to DMF in vitro (Thiessen et al. 2010). Neurons treated with DMF showed an Nrf2-dependent upregulation of their antioxidant response and showed better in vitro survival after toxic oxidative challenge (Scannevin et al. 2012). Furthermore, an upregulation of glutathione and other antioxidant enzymes in oligodendrocytes has been shown when they were incubated with DMF in vitro (Albrecht et al. 2012). In summary, DMF and MMF exert evident antioxidative effects on CNS-resident cells.

\subsubsection{Immunomodulatory effects}

Next to its antioxidative function, DMF has also strong modulating effects on the peripheral immune system: In vitro studies showed that DMF reduces the chemokine (C-C motif) ligand-2-induced chemotaxis of human monocytes, which potentially leads to a decreased CNS infiltration (Cross et al. 2011). It has been further demonstrated that DMF inhibits the production of pro-inflammatory cytokines, such as IL-1 $\beta$, IL-6 and TNF via activated microglia and astrocytes (Wilms et al. 2010). Moreover, data indicate that the decreased production of TNF upon DMF treatment results in a significant downregulation of adhesion molecules such as intracellular adhesion molecule 1, vascular cell adhesion molecule 1 and E-selectin on the endothelium, resulting in an impairment of dynamic lymphocyteendothelial cell interactions in vitro (Wallbrecht et al. 2011).

Several studies elucidated that DMF and MMF are capable of binding the intracellular nuclear factor 'kappa-light-chain-enhancer' of activated B-cells (NF- $x$ B). In doing so, they inhibit the translocation of this protein into the nucleus and its binding to the deoxyribonucleic acid. This affects the cell maturation and leads to a reduced anti-inflammatory cytokine production as well as an altered antigen-presenting function (Gillard et al. 2015; Litjens et al. 2006; Loewe et al. 2002). In addition, it has been demonstrated that DMF inhibits toll-like receptor(TLR)induced M1 and K63 ubiquitin formation and thereby blocks pro-inflammatory cytokine production (McGuire et al. 2016).

Furthermore, it was shown that the enzyme glyceraldehyde 3-phosphate dehydrogenase $(\mathrm{GAPDH})$ is succinated and inactivated by the MMF (Kornberg et al. 2018). Thereby, aerobic glycolysis of activated myeloid and lymphoid cells with a high metabolic turnover is thought to be downregulated, mediating the anti-inflammatory effects of DMF.

Animal experiments showed that DMF treatment reduces the infiltration of macrophages into the spinal cord of EAE-affected mice (Linker et al. 2011). This is possibly mediated by its active metabolite MMF, which is assumed to be a potent agonist of HCAR2, a receptor 
that is mostly expressed on neutrophils, but also other immune cells (Lukasova et al. 2011). By impairing the neutrophil recruitment, DMF may exert a secondary immunomodulatory function leading to less infiltration (Chen et al. 2014). Supporting this hypothesis, another study showed that in EAE mice, beneficial effects on microglia seem to be also HCAR2-dependent and to rescue synaptic dysregulation in the inflamed CNS (Parodi et al. 2015).

As MS was traditionally considered a T cell-driven disease, first studies regarding the effect of DMF on the peripheral immune system of MS patients were performed with a focus on $\mathrm{T}$ cells. These experiments with in vitro DMF-treated mononuclear blood cells revealed that DMF fosters a $T_{h} 2$ cell response (Jong et al. 1996). A few years later, it was shown that MMF increases the production of IL-4 and IL- 5 of human monocyte-derived dendritic cells, which are known to foster $T_{h} 2$ cell development and down-regulate $T_{h} 1$ cell responses (Litjens et al. 2004). Along the same line, it was demonstrated that DMF stimulates the development of Type II dendritic cells that produce anti-inflammatory IL-10. Those cells in turn are able to induce IL-4 secreting $T_{h} 2$ cells both in vivo and in vitro (Ghoreschi et al. 2011). The shift towards $\mathrm{T}_{\mathrm{h}} 2$ cells that was seen in all these experiments could be confirmed in human blood samples of patients treated with DMF (Gross et al. 2016). Besides the overall reduction in the absolute count of circulating lymphocytes upon DMF treatment (Gold et al. 2017), it has been reported that DMF causes a specific reduction of $\mathrm{CD}^{+} \mathrm{T}$ cells, resulting in a shift towards an increasing frequency of $\mathrm{CD}^{+}{ }^{+} \mathrm{T}$ cells (Spencer et al. 2015). These $\mathrm{CD} 4^{+}$cells additionally showed a rather anti-inflammatory cytokine profile while the frequency of follicular $T_{h}$ cells was reduced upon DMF treatment (Holm Hansen et al. 2018; Tahvili et al. 2015). Furthermore, memory $T$ cell counts appear to be significantly diminished upon treatment (Spencer et al. 2015; Longbrake and Cross 2016). Another study suggests that DMF is a potent inducer of apoptosis in human T cells in vitro (Treumer et al. 2003). In summary, T cell-mediated inflammation seems to be strongly inhibited by DMF treatment.

DMF treatment does not affect the absolute number of monocytes in the blood, but due to deceasing leukocyte numbers, there is a relative increase of the monocyte frequency. Furthermore, DMF was shown to reduce the monocytic production of pro-inflammatory cytokines such as IL-6 and TNF and increased the apoptosis rate of human monocytes (Michell-Robinson et al. 2016).

B lymphocytes are assumed to play a key role in the pathogenesis of MS by their two main functions cytokine secretion and antigen presentation (Lund 2008). However, at the beginning of this data gathering, not much was known about the impact of DMF on B cells.

\subsection{Aims of the study}

Even though DMF is frequently prescribed for treatment of RRMS and more than 100,000 patients worldwide have taken it since 2013, DMF's exact mechanism of action is still not 
fully understood. As MS was long time considered a mainly T cell-driven disease, many data have been collected focusing on the effect of DMF on these cells. However, whether and how it influences $B$ cells, which were newly recognized key player in the immunopathogenesis of MS, where they serve as APC and source of pro-inflammatory cytokines, is still elusive.

Therefore, the current study was performed to gain a deeper understanding how DMF treatment influences B cells and their disease-driving functions in a more detailed manner: Peripheral blood mononuclear cells (PBMC) of DMF-treated patients and controls were acquired to investigate different aspects of DMF-mediated effects, especially on B cells. To understand whether DMF alters the general competition of PBMC, major immune cell frequencies such as T cells, CD14 ${ }^{+}$phagocytes and B cells were determined. Next, with more detailed focus on $\mathrm{B}$ lymphocytes, different maturation stages of $\mathrm{B}$ cell development were assessed. B cells can either be activated unspecifically by cytokines and chemokines secreted by other immune cells or pathogen-associated molecular pattern (PAMP) binding to toll-like receptors or specifically by the binding of antigen to the $\mathrm{B}$ cells receptor. Hence, this study analyzed the expression of certain surface molecules (CD25, CD69, CD95 and CD150) involved in cell interaction and signaling. For antigen presentation, APC internalize molecules either randomly (myeloid cells) or specifically (B cells), process them and present linearized peptides to responding $\mathrm{T}$ cells. This cell-cell contact is then further stabilized by the interaction of co-stimulatory molecules and the exchange of secretory signals. The nature of these signals determines the fate of the responding $\mathrm{T}$ cells and shapes their differentiation. That's why CD40, CD80, CD86 and MHC-II were measured in this investigation. Lastly, B cells and myeloid-derived phagocytes can influence other immune cells such as T cells by the secretion of diverse cytokines and chemokines. These soluble factors bind to receptors on $\mathrm{T}$ cells and thereby trigger a cascade of effects and functions determining their differentiation. Major pro- (TNF and IL-6) and anti-inflammatory (IL-10) cytokines were evaluated to understand the effect of DMF in this field. 


\section{Materials and Methods}

\subsection{Patients}

Blood samples were taken from patients of the Göttingen University Medical Center in Germany between 2015 and 2017 with confirmed RRMS. The diagnosis was based on the McDonald criteria. Treated patients were taking DMF (Tecfidera) for at least 3 months when phlebotomy was performed. In the aggregate, 30 control MS patient samples were compared to 31 samples of MS patients taking DMF. Six patients were analyzed longitudinal, meaning that blood samples were taken before and during treatment. In addition, demographic and disease-related information was collected from all patients. All participants provided informed consent prior to study enrollment as approved by the Göttingen University ethics review board (\#3/4/14).

Table 2: Demographic and disease characteristics of the patients. (DMF = dimethyl fumarate; $\mathrm{y}=$ years; $\mathrm{SD}=$ standard deviation; EDSS = extended disability status scale; MS = multiple sclerosis)

\begin{tabular}{|l|cc|c|}
\hline & \multicolumn{2}{|c|}{ horizontal } & $\begin{array}{c}\text { longitudinal } \\
\text { (switch to DMF) }\end{array}$ \\
\hline Number of patients & control & DMF & 6 \\
Age [y] (mean \pm SD) & 24 & 25 & $34.3 \pm 9.4$ \\
Female sex [\%] & $37.2 \pm 9.8$ & $35.4 \pm 11.1$ & 50.0 \\
EDSS score (mean \pm SD) & 75.0 & 48.0 & $1.8 \pm 1.3$ \\
MS since [y] (mean \pm SD) & $2.2 \pm 2.0$ & $1.4 \pm 1.2$ & $10.8 \pm 7.4$ \\
DMF since [m] (mean \pm SD) & $7.7 \pm 10.0$ & $3.0 \pm 3.2$ & $-/ 7.6 \pm 2.2$ \\
Previous treatment [cases]: & - & $12.8 \pm 7.5$ & 0 \\
$\quad$ Natalizumab & 2 & & 1 \\
\multicolumn{1}{|c|}{ IFN- $\beta$} & 5 & 5 & 2 \\
Glatiramer acetate & 2 & 6 & \\
\hline
\end{tabular}




\subsection{Materials}

Table 3: Human monoclonal antibodies for flow cytometry. $(\mathrm{CD}=$ cluster of differentiation; MHC-II = major histocompatibility complex - class II; IL = interleukin; TNF = tumor necrosis factor)

\begin{tabular}{|c|c|c|c|c|}
\hline Antigen & Fluorochrome & Clone & Dilution & $\begin{array}{l}\text { Manufacturing } \\
\text { company }\end{array}$ \\
\hline CD4 & PE-Cy7 & RPA-T4 & $1: 100$ & BD Biosciences \\
\hline CD8 & PE & HIT-8a & 1:100 & eBioscience \\
\hline CD14 & BV421 & M5E2 & $1: 100$ & BD Biosciences \\
\hline CD14 & FITC & M5E2 & 1:100 & BD Biosciences \\
\hline CD14 & PE-CF594 & $\mathrm{M} \varphi \mathrm{P} 9$ & $1: 100$ & BD Biosciences \\
\hline CD19 & APC & HIB19 & 1:100 & BD Biosciences \\
\hline CD19 & FITC & HIB19 & 1:100 & BD Biosciences \\
\hline CD19 & PerCp-Сy5.5 & HIB19 & 1:100 & BioLegend \\
\hline CD19 & PE-Cy5 & HIB19 & 1:100 & BD Biosciences \\
\hline $\mathrm{CD} 20$ & APC-Cy7 & L27 & 1:100 & BD Biosciences \\
\hline $\mathrm{CD} 24$ & PerCp-Cy5.5 & ML5 & 1:100 & BioLegend \\
\hline CD25 & BV605 & ВС96 & 1:100 & BioLegend \\
\hline CD27 & PacificBlue & O323 & 1:100 & BioLegend \\
\hline CD38 & FITC & HIT2 & 1:100 & BioLegend \\
\hline CD40 & PE/Dazzle & $5 \mathrm{C} 3$ & 1:100 & BioLegend \\
\hline CD69 & FITC & FN50 & 1:100 & BioLegend \\
\hline CD80 & PE-Cy7 & L307.4 & 1:100 & BD Biosciences \\
\hline CD86 & BV421 & FUN-1 & 1:100 & BD Biosciences \\
\hline CD95 & $\mathrm{PE}$ & DX2 & 1:100 & BioLegend \\
\hline CD150 & BV421 & A12 & 1:100 & BD Biosciences \\
\hline MHC-II & APC & Tü36 & 1:100 & BioLegend \\
\hline IL-6 & FITC & MQ2-13A5 & $1: 50$ & BD Biosciences \\
\hline IL-10 & PE-CF594 & JES3-19F1 & $1: 50$ & BD Biosciences \\
\hline TNF & A700 & MAb11 & $1: 50$ & BD Biosciences \\
\hline Dead/Live & ZombieAqua $^{\text {TM }}$ & & $1: 100$ & BioLegend \\
\hline Dead/Live & ZombieNIR $^{\mathrm{TM}}$ & & 1:100 & BioLegend \\
\hline
\end{tabular}


Table 4: Reagents, proteins and inhibitors.

\begin{tabular}{|c|c|}
\hline & Company \\
\hline BSA (bovine serum albumin) & SERVA GmbH \\
\hline BD fluorescence-activated cell sorting (FACS) Clean ${ }^{\mathrm{TM}}$ & BD Biosciences \\
\hline BD FACS Flow ${ }^{T M}$ & BD Biosciences \\
\hline BD FACS Rinse ${ }^{\mathrm{TM}}$ & BD Biosciences \\
\hline BioColl separation solution & Biochrom \\
\hline Cytosine-phosphate-Guanine (CpG) oligodeoxynucleotides & Sigma Aldrich \\
\hline Cytofix/Cytoperm ${ }^{\mathrm{TM}}$ & BD Biosciences \\
\hline DMEM (Dulbecco's Modified Eagle's medium) & Sigma Aldrich \\
\hline DMSO (dimethyl sulfoxide) & Sigma Aldrich \\
\hline EDTA (ethylene diamine tetraacetic acid disodium salt dihydrate) & Carl Roth \\
\hline Ethanol 100\% & Merck Millipore \\
\hline Fc-block ${ }^{\mathbf{T M}}$ & BioLegend \\
\hline FCS (fetal calf serum) & Sigma Aldrich \\
\hline GolgiPlugTM & BD Biosciences \\
\hline $\mathrm{H}_{2} \mathrm{SO}_{4}$ (sulfuric acid) $97 \%$ & Merck Millipore \\
\hline Ionomycin & Sigma Aldrich \\
\hline LPS (lipopolysaccharides) & Sigma Aldrich \\
\hline $\mathrm{NaCO}_{3}$ (sodium carbonate) & Merck Millipore \\
\hline $\mathrm{NaHCO}_{3}$ (sodium hydrogen carbonate) & Merck Millipore \\
\hline PBS (phosphate buffered salt solution) & Sigma Aldrich \\
\hline Perm/Wash ${ }^{\text {TM }}$ buffer, $10 \mathrm{x}$ & BD Biosciences \\
\hline Paraformaldehyde & Merck Millipore \\
\hline PMA (phorbol 12-myristrate 13-acetate) & Sigma Aldrich \\
\hline RPMI-1640 (Roswell Park Memorial Institute-1640) & Sigma Aldrich \\
\hline Sodium pyruvate $100 \mathrm{mM}$ & Sigma Aldrich \\
\hline TMB (tetramethylbenzidine) & eBioscience \\
\hline TrypanBlue & SigmaAldrich \\
\hline Tween & Merck Millipore \\
\hline$\beta$-mercaptoethanol & Sigma Aldrich \\
\hline
\end{tabular}


Table 5: Solutions, buffers and cell culture media. (RPMI = Roswell Park Memorial Institute-1640; FCS = fetal calf serum; DMSO = dimethyl sulfoxide; ELISA = enzyme-linked immunosorbent assay; $\mathrm{PBS}=$ phosphate-buffered salt solution; BSA = bovine serum albumin)

\begin{tabular}{|l|l|}
\hline Solution & Composition \\
\hline Cryo-medium & $60 \% \mathrm{RPMI}_{\text {complete }} 20 \% \mathrm{FCS}, 20 \%$ DMSO \\
ELISA blocking buffer & 1.81 distilled water, $200 \mathrm{ml}$ PBS 10x, $20 \mathrm{~g}$ BSA \\
ELISA coating buffer & 11 distilled water, $8.4 \mathrm{~g} \mathrm{NaHCO}, 3.5 \mathrm{~g} \mathrm{NACO}_{3}$ \\
ELISA stop solution & $1 \mathrm{M} \mathrm{H}_{2} \mathrm{SO}_{4}$ solution \\
ELISA wash buffer & 1.81 distilled water, $200 \mathrm{ml}$ PBS $10 \mathrm{x}, 1 \mathrm{ml}$ Tween \\
FACS buffer $^{2 \% ~ F C S ~ i n ~ P B S ~}$ \\
RPMI $_{\text {complete }}$ & $0.51 \mathrm{RPMI}-1640,50 \mathrm{ml} \mathrm{FCS,} 5 \mathrm{ml}$ sodium pyruvate, $5 \mathrm{ml} \mathrm{L-}$ \\
& glutamine, $0.5 \mathrm{ml} \beta$-mercaptoethanol \\
\hline
\end{tabular}

Table 6: Consumables. $\quad$ FACS $=$ fluorescence-activated cell sorting; ELISA $=$ enzyme-linked immunosorbent assay)

\begin{tabular}{|l|l|}
\hline Product & Manufacturing company \\
\hline 96 well plates, flat bottom & Sarstedt \\
96 well plates, round bottom & Sarstedt \\
FACS tubes, $5 \mathrm{ml}$ & Sarstedt \\
Micro tubes $(0.1 \mathrm{ml}, \mathbf{0 . 5} \mathrm{ml}, \mathbf{1} \mathrm{ml}, \mathbf{1 . 5} \mathrm{ml})$ & Eppendorf \\
Multistep pipettes, $1 \mathrm{ml}$ & Eppendorf \\
NunctM 96 well ELISA plates & ThermoScientific \\
Pipettes $(10 \mu 1,200 \mu 1,1000 \mu l)$ & Sarstedt \\
Pipettes $(5 \mathrm{ml}, 10 \mathrm{ml}, \mathbf{2 5} \mathrm{ml})$ & Sarstedt \\
\hline
\end{tabular}


Table 7: Technical devices.

\begin{tabular}{|l|l|}
\hline Device & Manufacturing company \\
\hline BBD 620 cell incubator & ThermoScientific \\
Centrifuge 5415R & Eppendorf \\
Centrifuge 5810R & Eppendorf \\
CKX41 light microscope & Olympus \\
FACS LSRII Fortressa & BD Biosciences \\
IMARK & Bio-Rad \\
Neubauer chamber & Superior Marienfeld \\
SAFE 2020 clean bench & ThermoScientific \\
\hline
\end{tabular}

Table 8: Software.

\begin{tabular}{|l|l|}
\hline Software & Company \\
\hline Microplate Manager $\mathbf{6}^{\mathbf{T M}}$ & Bio-Rad \\
FACSdiva $^{\text {TM }}$ 6.1.2 & BD biosciences \\
FlowJo $^{\text {TM }} 10.2$ & Tree Star Inc. \\
GraphPad Prism $^{\text {TM }} \mathbf{6 . 0 1}$ & GraphPad software Inc. \\
\hline
\end{tabular}

\subsection{Methods}

PBMC isolation, sample processing, cryopreservation and subsequent unfreezing were performed according to established protocols in the laboratory.

\subsubsection{Preparation of PBMC}

Patients' blood samples (each $30 \mathrm{ml}$ ) were taken by phlebotomy directly into tubes containing EDTA to avoid cell agglutination. Thereafter, the blood was transferred into sterile tubes and diluted 3:1 with PBS. To isolate peripheral blood mononuclear cells, this blood-PBS suspension was given carefully onto a $20 \mathrm{ml}$ BioColl ${ }^{\mathrm{TM}}$ layer and centrifuged for 35 minutes at $448 \mathrm{~g}$ and $21{ }^{\circ} \mathrm{C}$ with low acceleration and no brake. After centrifugation, PBMC were concentrated in the interphase between BioColl and the soluble compounds of the blood, visible as a white layer, which were transferred into a new tube. To remove undesirable residues, the cells were diluted twice with DMEM followed by centrifugation for 10 minutes at 1250 revolutions per minute $(\mathrm{rpm})$ and $4{ }^{\circ} \mathrm{C}$. Thereafter, cells were resuspended in $5 \mathrm{ml}$ 
DMEM and the cell number was determined as described below. For cryo-conservation, cells were diluted to a density of $4 \times 10^{6} \mathrm{ml}^{-1}$ in DMEM. Each cryo-tube was filled with $500 \mu \mathrm{l}$ of the suspension and $500 \mu \mathrm{l}$ cryo-medium to achieve a total cell count of 2 million cells per tube. Using an ethanol cooling box, that allows a slow cooling rate of $1^{\circ} \mathrm{K}$ per minute, the cryo-tubes were frozen and stored in a $-80{ }^{\circ} \mathrm{C}$ freezer until further preparation.

\subsubsection{Flow cytometric staining panels and stimulation regime}

To examine the above mentioned aims of the study, different staining panels and stimulation regimes had to be established. This was necessary because there is a technical limit of the number of different fluorophores that can be used on the same cells without a too pronounced spectral overlap. This overlap would lead to undesired unspecific signals in the different detectors. Thus, the developed panels were tested and optimized before the patients' samples were measured. In addition, spectral analysis of the distinct fluorophore constellations was conducted. The resulting panels are shown in Table $\mathbf{9}$ and 10:

Table 9: Staining panels (1). (CD = cluster of differentiation; MHC-II = major histocompatibility complex - class II; IL = interleukin; TNF = tumor necrosis factor)

\begin{tabular}{|c|c|c|}
\hline Cell distribution (a) & B cell subsets (b) & Cytokine production (c) \\
\hline CD4- PE-Cy7 & CD19- APC & CD14- BV421 \\
CD8- PE & CD20- APC-Cy7 & CD19- PE-Cy5 \\
CD14- PE-CF594 & CD24- PerCp-Cy5.5 & IL-6 - FITC \\
CD19- FITC & CD27- PacificBlue & IL-10- PE-CF594 \\
CD150- BV421 & CD38- FITC & TNF - A700 \\
MHC-II - APC & CD95- PE & ZombieNIR \\
ZombieAqua & ZombieAqua & \\
\hline
\end{tabular}


Table 10: Staining panels (2). (CD = cluster of differentiation; MHC-II = major histocompatibility complex - class II; IL = interleukin; TNF = tumor necrosis factor)

\begin{tabular}{|c|c|}
\hline Activation markers (d) & Antigen-presenting function (e) \\
\hline CD19- PerCp-Cy5.5 & CD14 - FITC \\
CD25- BV605 & CD19- PerCp-Cy5.5 \\
CD40- PE/Dazzle & CD40- PE/Dazzle \\
CD69- FITC & CD80- PE-Cy7 \\
CD80- PE-Cy7 & CD86- BV421 \\
CD86- BV421 & MHC-II - APC \\
MHC-II - APC & CD95- PE \\
CD95 - PE & ZombieNIR \\
ZombieNIR & \\
\hline
\end{tabular}

As some activation markers and cytokines are expressed or synthesized only upon stimulation, cells in some panels were stimulated and incubated before the staining took place. The following four substances were used for this:

The $\mathbf{C p G}$ dinucleotide has a high abundance in the microbial genome and is recognized by immune cells as a PAMP. Through the TLR-9 receptor, it promotes antibody and cytokine production.

Lipopolysaccharides (LPS) are found on the outer membrane of gram-negative bacteria. Therefore, immune cells produce pro-inflammatory cytokines when LPS stimulates them through a TLR-4 receptor NF- $x \mathrm{~B}$ pathway.

Ionomycin is an ionophore produced by the bacterium Streptomyces conglobatus. It enhances cellular calcium influx and makes cells produce more cytokines.

The tumor promoter phorbol 12-myristate 13-acetate (PMA) also upregulates cytokine production via a protein kinase $\mathrm{C}$ pathway.

The developed stimulation regimes are presented in Table 11. 
Table 11: Stimulation regimes. (LPS $=$ lipopolysaccharide; $\mathrm{h}=$ hours; $\mathrm{CpG}=$ cytosine-phosphate-guanine; PMA = phorbol 12-myristate 13-acetate)

\begin{tabular}{|l|l|}
\hline Staining panel & Stimulation regime \\
\hline Cell distribution (a) & No stimulation \\
B cell subsets (b) & No stimulation \\
Antigen-presenting function (e) & No stimulation \\
Cell distribution (a) & $100 \mathrm{pg} / \mathrm{ml} \mathrm{LPS} \mathrm{for} 20 \mathrm{~h}$ \\
Activation markers (d) & $2 \mu \mathrm{g} / \mathrm{ml} \mathrm{CpG} \mathrm{for} 20 \mathrm{~h}$ \\
Cytokine production (c) & $1 \mu \mathrm{g} / \mathrm{ml} \mathrm{CpG} \mathrm{for} 20 \mathrm{~h}$, then + GolgiPlug, after $2 \mathrm{~h}$ \\
& $+500 \mathrm{ng} / \mathrm{ml}$ ionomycin and $20 \mathrm{ng} / \mathrm{ml}$ PMA for $2 \mathrm{~h}$ \\
\hline
\end{tabular}

The following surface molecules were assessed to evaluate $\mathbf{B}$ cell activation:

CD25 is a type I transmembrane glycoprotein and can be found on activated mononuclear immune cells. It associates with CD122 and CD132 to form the IL-2 receptor complex. The MS drug daclizumab binds and blocks this protein (Leonard et al. 1985).

CD27 is a type I membrane protein that binds CD70 and plays a role in T cell co-stimulation and $\mathrm{B}$ cell maturation. It was shown to be part of the TNF receptor-associated factor 2 (TRAF2) NF- $x$ B pathway (Akiba et al. 1998). SIVA, a pro-apoptotic protein, can bind CD27 and may induce apoptosis (Prasad et al. 1997).

CD69 is a type II transmembrane protein and member of the C-type lecin family. It is involved in early activation processes of lymphocytes. Furthermore, it functions as a signaltransmitting receptor in platelets, natural killer (NK) cells and lymphocytes (Cambiaggi et al. 1992).

CD95 is a so-called death receptor and additionally known as FAS receptor or apoptosis antigen 1. It is a member of the TNF receptor superfamily and upregulated in activated T and B lymphocytes. Binding FAS ligand (CD178) induces a trimerization and consequently the formation of the death-inducing signal complex. This leads to the apoptosis of the cell and has been shown to play a role in the maintenance of peripheral tolerance. Thus, defects in CD95 might be involved in the development of autoimmune diseases. The expression of CD95 is regulated by NF-xB (Liu et al. 2012).

CD150, also known as signaling lymphocytic activation molecule, is a type I transmembrane molecule that is also a member of the Ig superfamily. It is expressed on stimulated B cells, T cells, dendritic cells and endothelial cells. It can bind itself as ligand and is involved in B cell proliferation, co-stimulation, immunoglobulin production and signal transduction (Howie 2002). 
The ability of $\mathrm{B}$ cells and $\mathrm{CD} 14^{+}$phagocytes to present antigen was assessed with the following key molecules:

MHC-II is found on any antigen-presenting cell and crucial for the initiation of immune responses. After internalization of an external protein, APC digest the phagocyted molecules to linearized peptides inside their phagolysosomes. Subsequently, they are loaded onto MHC-II molecules and presented via MHC-II on the cell surface. Antigen-specific CD4 ${ }^{+} \mathrm{T}$ cells recognize these short peptides via their $\mathrm{T}$ cell receptor.

Being a type I glycoprotein, CD40 has been reported to be involved in B cell differentiation, co-stimulation, isotype class-switching and protection of B cells from apoptosis. It is a member of the TNF receptor superfamily and important for the B cell - T cell interaction by binding CD40 ligand on T cells (Pype et al. 2000).

CD80, also known as B7-1 is mainly expressed on APC such as activated B cells, macrophages and dendritic cells. It belongs to the immunoglobulin superfamily and forms a type I glycoprotein. By interacting with $\mathrm{CD} 28$ and $\mathrm{CD} 152$ (cytotoxic $\mathrm{T}$ lymphocyte-associated protein 4 (CTLA-4)), it plays a crucial role in the regulation of the T cell activation. While binding CD28 provides a strong costimulatory signal, the interaction with CTLA-4 inhibits T cell activation (Bhatia et al. 2006).

CD86 is a type I glycoprotein, works in tandem with CD80 to prime T cells and is also known as B7-2. Similar to CD80 it is expressed on antigen-presenting cells and interacts with CD28 and CD152 (CTLA-4) on T cells providing co-stimulatory or inhibitory signals, respectively. Its expression occurs earlier than CD80 and it was shown to be involved in immunoglobulin class-switching (Chen et al. 1994).

To evaluate B cell function in further detail, the following cytokines were assessed:

IL-6 is also called lymphocyte stimulating factor or IFN- $\beta 2$. As a pro-inflammatory protein, it plays an important role in the interaction between the initial and the acquired immune response. IL-6 supports B cell growth and activation. Activated B cells themselves produce IL-6 and further foster the inflammatory process, especially by generating $T_{h} 17$ cells, which are relevant is the pathophysiology of MS (Hunter and Jones 2015). In line, it has been documented that in MS patients, B cells secrete increased levels of IL-6 (Bar-Or et al. 2010).

TNF is the most prominent member of the TNF superfamily, it is mainly secreted by macrophages, but also by lymphocytes, endothelial cells and others. It enhances the chemotaxis of granulocytes and stimulates the phagocytosis of macrophages. Thus, it is considered a pro-inflammatory cytokine. Binding TNF receptor 1 , it has similar downstream effects as IL-6, mediated by TRAF2 and NF- $x \mathrm{~B}$. This cytokine is important for the current study as B cells of MS-patients were shown to produce high levels of TNF (Bar-Or et al. 2010). 
IL-10 is considered an anti-inflammatory cytokine. Therefore, it was called cytokinesynthesis inhibitory factor. Main producer of IL-10 are monocytes, but to a smaller extend it is also synthesized by regulatory $\mathrm{T}$ and B lymphocytes. In patients with MS, IL-10 levels are reduced (Duddy et al. 2007). Its production is highly regulated and may depend of MAPK1/2, p38 and NF- $x \mathrm{~B}$ signaling. Autoregulation is also presumed. When IL-10 is binding to the IL-10 receptor complex, it has multiple effects on the downstream pathway. It suppresses the expression of pro-inflammatory cytokines, MHC-II and co-stimulatory molecules on macrophages (Mosser and Zhang 2008).

\subsubsection{Sample preparation}

The frozen cryo-tubes containing $2,000,000$ cells each were stored at $-80{ }^{\circ} \mathrm{C}$. For quick and safe thawing of the samples they were put into a $37^{\circ} \mathrm{C}$ water quench for 30 seconds. As the cyro-medium is toxic for unfrozen cells, they were transferred into $40 \mathrm{ml}$ RPMI immediately after thawing. After centrifugation $\left(1250 \mathrm{rpm}, 4^{\circ} \mathrm{C}, 10 \mathrm{~min}\right)$, the supernatant was removed and the sedimented cells were resuspended in $20 \mathrm{ml}$ RPMI followed by a second centrifugation. Being resuspended in $1 \mathrm{ml} \mathrm{RPMI}$, the cell number was determined.

Therefore, a Neubauer chamber was used. To exclude dead cells, $10 \mu \mathrm{l}$ of cell suspension was diluted in $40 \mu \mathrm{l}$ or $90 \mu \mathrm{l}$ TrypanBlue (prediluted 1:10 in PBS), resulting in a dilution factor (dil. fac.) of 5 or 10. Subsequently, $10 \mu$ l of the mixture was given onto the counting chamber. Cells on four different $1 \mathrm{~mm}^{2}$ squares, representing a volume of $0.1 \mu \mathrm{l}$ each, were counted. Hereby the total cell number could be calculated using the following formula:

$$
\text { Total cell count }=\frac{\text { Counted cells }}{4} * 10000 * \text { dil.fac. } * \text { volume of suspension }[\mathrm{ml}]
$$

Before cell staining, the cell suspension was then diluted with RPMI to a concentration of $2 \times 10^{6}$ cells $/ \mathrm{ml}$. A total of 200,000 cells $(100 \mu$ l of the prepared cell suspension) were added into the single wells of a 96 well plate. If cells were not stimulated (panels a, b, e) U-bottom 96-well plates were used and samples were directly stained as described below (2.3.4).

In order to stimulate the expression of activation markers and the production of cytokines (panel a, b, c), cells were plated in flat bottom 96-well plates. According to the stimulation regimes (Table 11), $2 \mu \mathrm{g} / \mathrm{ml} \mathrm{CpG}$ or $100 \mathrm{pg} / \mathrm{ml} \mathrm{LPS}$ were added to the cell suspension. These plates were then incubated at $37^{\circ} \mathrm{C}$ and $5 \% \mathrm{CO}_{2}$ for 20 hours. Supernatants were thereafter frozen at $-18{ }^{\circ} \mathrm{C}$ until further examination.

\subsubsection{Dead/Live staining}

To exclude dead cells from the analysis, cells were stained with fixable Zombie ${ }^{\mathrm{TM}}$ dyes (BioLegend), which react with primary amine groups on proteins. In living cells with an intact cell membrane, the dye only binds to surface proteins resulting in a low staining. The fact that the cell membrane of dead cells is much more permeable lets the dye enter and label all 
kinds of proteins within the cytoplasm. Subsequently, they appear much brighter in Zombie $^{\mathrm{TM}}$ fluorescence than living cells.

Before staining, cells were resuspended in $200 \mu \mathrm{l}$ PBS followed by centrifugation (1250 rpm, $\left.4{ }^{\circ} \mathrm{C}, 7 \mathrm{~min}\right)$. Thereafter, the supernatant was removed, $30 \mu \mathrm{l}$ of the Zombie dye (1:100 diluted in PBS) were given onto the cells, excluding non-Zombie single stains. The incubation time was $10 \mathrm{~min}$ at room temperature in the dark. Then the cells were washed in $200 \mu$ fluorescence-activated cell sorting (FACS) buffer.

\subsubsection{Surface staining}

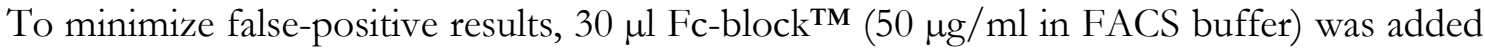
to the cells in each well. This inhibitor blocks non-specific binding of antibodies to Fc receptors on the cell surface. After an incubation of $10 \mathrm{~min}$ at $4{ }^{\circ} \mathrm{C}$ in the dark, surface staining could be performed. The fluorophore-labeled antibodies of each panel were diluted 1:100 in FACS buffer in the same micro tube. The resulting antibody-mix contained all fluorophores for one panel. Additionally, single antibodies were diluted 1:100 in FACS buffer.

$30 \mu \mathrm{l}$ of the antibody-mix were added to all patients' samples and single antibody solutions to the single stain wells. The following incubation in the dark lasted $15 \mathrm{~min}$ at $4{ }^{\circ} \mathrm{C}$. Thereafter, the cells were washed with $200 \mu \mathrm{l} \mathrm{FACS} \mathrm{buffer}$ and then re-suspended in $100 \mu \mathrm{l}$ FACS buffer. For all panels, excluding the intracellular staining of the cytokine, the cell suspension of each well was transferred into $5 \mathrm{ml} \mathrm{FACS}$ tubes.

\subsubsection{Intracellular staining}

To block the Golgi apparatus, $10 \mu$ GolgiPlug $^{\mathrm{TM}}$ (pre-diluted 1:10 in RPMI) was added to the CpG-stimulated cells after 20 hours (panel c). This is necessary to accumulate synthesized proteins including cytokines inside the cells, making them detectable by flow cytometry. To further enhance cytokine production, $500 \mathrm{ng} / \mathrm{ml}$ ionomycin and $20 \mathrm{ng} / \mathrm{ml}$ PMA was added to each well after 2 hours (Table 11). Following an incubation of additional 2 hours, the plate was centrifuged and $230 \mu$ supernatant of each well was transferred into a new 96-well plate and stored at $-18{ }^{\circ} \mathrm{C}$ until cytokine concentration was analyzed by enzyme-linked immunosorbent assay (ELISA). The plated cells in turn were resuspended in FACS buffer and cell surface staining was performed as described in 2.3.4 and 2.3.5.

For intracellular staining, the cell membrane was permeabilized by adding $100 \mu \mathrm{Fix} / \mathrm{Perm}^{\mathrm{TM}}$ per well after cell surface staining for 30 minutes at $4{ }^{\circ} \mathrm{C}$ in the dark. Thereafter, cells were washed with $200 \mu \mathrm{l} \mathrm{Perm} / \mathrm{Wash}^{\mathrm{TM}}$ per well. $50 \mu \mathrm{l}$ of the prepared intracellular antibody mix (pre-diluted 1:50 in Perm/Wash ${ }^{\mathrm{TM}}$ ) were put onto the cells. Because of the increased permeability, the fluorophore-labeled antibodies can get inside the cell and label the cytokines of interest. For optimal staining, cells were incubated for 18 hours at $4{ }^{\circ} \mathrm{C}$ in the dark. The next day, cells were washed twice in $200 \mu \mathrm{l} \mathrm{Perm} / \mathrm{Wash}^{\mathrm{TM}}$ followed by a single wash with 
$200 \mu \mathrm{l}$ FACS buffer. Resuspended in $100 \mu \mathrm{l}$ FACS buffer, each sample was transferred into a $5 \mathrm{ml} \mathrm{FACS}$ tube and stored at $4^{\circ} \mathrm{C}$ before evaluation.

\subsubsection{Flow cytometry}

The fluorophore-labeled cells were analyzed by flow cytometry using the LSRII Fortressa FACS machine by BD Biosciences. To improve the accuracy of the flow cytometric staining, all patients' samples were plated in duplicates. Additional cells were plated in order to perform single stainings of the fluorophores. Each sample was acquired for 65 seconds per tube with a maximum of 100,000 events recorded.

As not all samples could be measured at the same time, the procedure was repeated several times. The compensation created when acquiring the data was very similar in all panels and runs. This is shown below. To make the comparison between the different runs more valid, the same compensation was applied for all runs.

The obtained data were analyzed using the FlowJo 10.2 software. 


\subsubsection{Gating strategy}

To avoid false positive signals, only living cells were analyzed. Thus, pre-gating was performed as shown in Figure 1A.

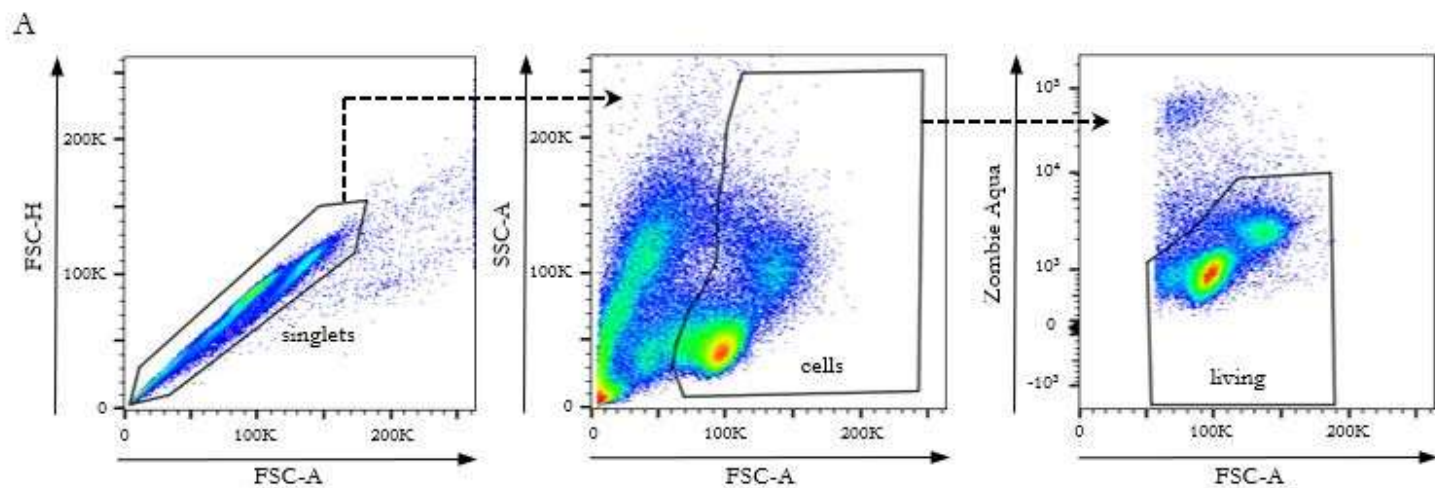

B

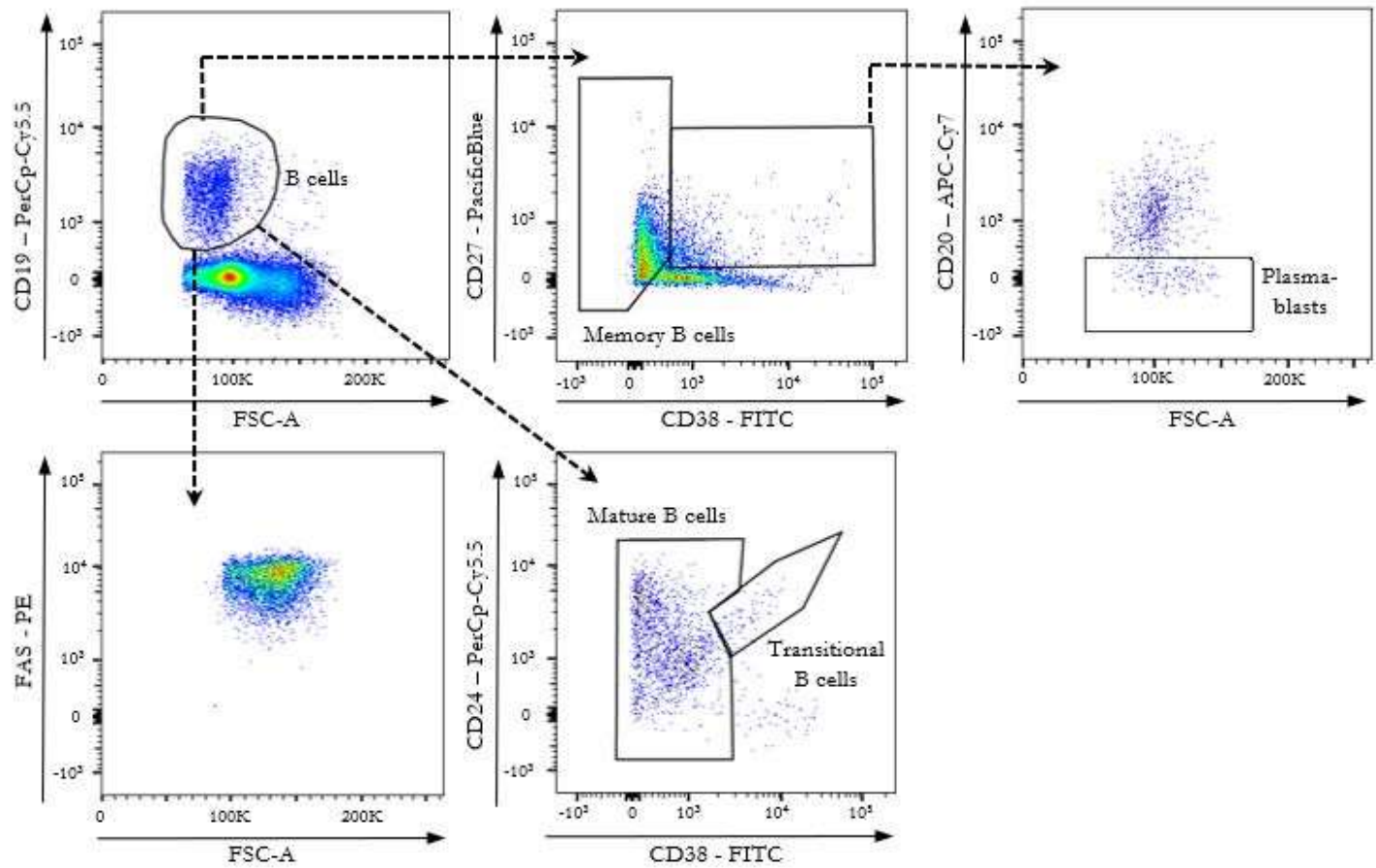

Figure 1: Pre-gating and gating strategy for B cell subsets and surface molecule expression. (A) Within all recorded events, singlets and cells were found using size exclusion (FSC = forward side scatter; SSC $=$ side scatter). Living cells were thereafter defined as Zombie-. (B) Within the living cells, the B cell gate was defined as cluster of differentiation (CD)19+. Surface marker were evaluated as mean fluorescence intensity. B cell subpopulations are defined as follows: memory B cells $\left(\mathrm{CD} 27^{\text {var }} \mathrm{CD} 38^{-}\right)$, plasmablasts $\left(\mathrm{CD} 20^{-} \mathrm{CD} 27^{+} \mathrm{CD} 38^{+}\right)$, mature B cells $\left(\mathrm{CD} 24\right.$ var $\left.\mathrm{CD} 38^{\text {low }}\right)$ and transitional B cells $\left(\mathrm{CD} 24^{\text {high }}\right.$ CD38high).

In the resulting pool of the living cells, the different cell types were defined according to their characteristic cell surface molecules. To quantify the expression of different cell surface markers, the mean fluorescence intensity (MFI) was used. In B cells, further subset gating was executed as shown in Figure 1B. 


\subsubsection{ELISA of supernatants}

To examine not only the frequency of cytokine-producing cells, but to also measure the amount of secreted cytokines, the concentration of IL-6, TNF and IL-10 was quantified in the frozen culture supernatants using ELISA. To bind the cytokine of interest to the cell surface of a 96-well ELISA plate, the plate was coated with capture antibody (BioLegend), according to the manufacturer's recommendation and incubated overnight at $4{ }^{\circ} \mathrm{C}$ in the dark. The next day, the plate was washed three times with ELISA wash buffer. To avoid unspecific binding, $200 \mu$ l ELISA blocking buffer were added and the covered plate was put on a cycler for 1 hour for incubation. During incubation time, standard samples and patient samples were prepared according to the manufacturer's recommendation. Thereafter, the plate was washed three times to remove unbound proteins and $30 \mu \mathrm{l}$ of the samples were added. After an incubation of 2 hours, the plate was washed to remove unbound components and ELISA detection antibody solution (BioLegend) was added to each well and incubated for 1 hour according to the manufacturer's recommendation. After washing the plate with ELISA wash buffer, $100 \mu \mathrm{l}$ Strept/Avidin solution (BioLegend) was added to each well and incubated for 30 minutes following the manufacturers' recommendation. To remove unbound Strept/Avidin the plate was washed three times and $100 \mu \mathrm{l} \mathrm{3,} \mathrm{3,} \mathrm{5,} \mathrm{5'}$ tetramethylbenzidine (TMB) was added to each well. The reaction was stopped by adding $100 \mu \mathrm{l}$ of ELISA Stop after 20 minutes, changing the color from blue to yellow. For data analyses, iMark microplate reader and software was used.

\subsubsection{Normalization}

To make the obtained data even more precise, the same control blood was analyzed at each measurement cycle. As there is an unavoidable fluctuation between all runs, normalization was performed. Therefore, all values measured by the MFI of the cells were normalized to the mean of the control values by using the following formula:

$$
M F I_{n}=M F I_{\text {normalized }}=M F I_{\text {aquired }} * \frac{M F I_{\text {control }}}{\text { mean MFI(all controls })}
$$

\subsubsection{Statistical analysis}

The data of the patient groups were tested for Gauss distribution using the D'Agostino \& Pearson omnibus normality test, the Shapiro-Wilk normality test and the KolmogorovSmirnov normality test. For the comparison of two cross-sectional groups with Gauss distribution an unpaired t-test was performed; for longitudinal samples the paired t-test. If the data were not Gauss distributed, a Mann Whitney test was applied in the cross-sectional analysis and the Wilcoxon matched-pairs signed rank test was used for the longitudinal data. GraphPad Prism ${ }^{\mathrm{TM}} 6$ was used for all statistical analysis. A p value $<0.05$ was considered statistically significant. For the testing of possible correlations, linear regression was used. 


\section{$3 \quad$ Results}

\subsection{Development of methods}

For this study, several flow cytometric measurements were performed. This method is based on the mechanism that fluorescence-labeled antibodies bind their specific antigen on the cell surface or inside a cell. Thus, multiple antigens can be determined on one single cell. The FACS machine then acquires the labeled cells separately, allowing single cell analysis. This provides an antigen profile of every cell. As multiple fluorophores are combined, this method allows detecting several antigens on the surface of a single cell. Hence, cell populations, cell activation and cytokine production can be characterized. Before the measurement of the patients' samples was started, the optimal stimulation regimes, staining compositions and read-out strategies had to be established. Therefore, different pre-experiments were performed. The following results describe the most relevant assays to achieve the optimal procedure.

\subsubsection{CpG/LPS titration essays}

Some antigens are only upon activation expressed on B cells and myeloid-derived phagocytes. Therefore, the optimal stimulation for a reliable detection of these markers had to be established. As B cells express both TLR-9, binding CPG, and TLR-4, binding LPS, those stimulants were tested. To find the optimal concentration, PBMC of healthy donors were incubated with different concentrations of CpG or LPS and the expression of defined activation markers on $\mathrm{B}$ cells and $\mathrm{CD} 14^{+}$cells were assessed. The time of stimulation was not altered in this setting as 20 hours of stimulation turned out to be most suitable.

As shown in Figure 2, all examined activation markers were upregulated at a concentration of $10 \mu \mathrm{g} / \mathrm{ml} \mathrm{CpG}$ and most of them at $1 \mu \mathrm{g} / \mathrm{ml} \mathrm{CpG}$. That is why for all future experiments, a CpG concentration of $2 \mu \mathrm{g} / \mathrm{ml}$ was chosen, being a compromise between the necessity of an adequate upregulation and the avoidance of a ceiling effect. 

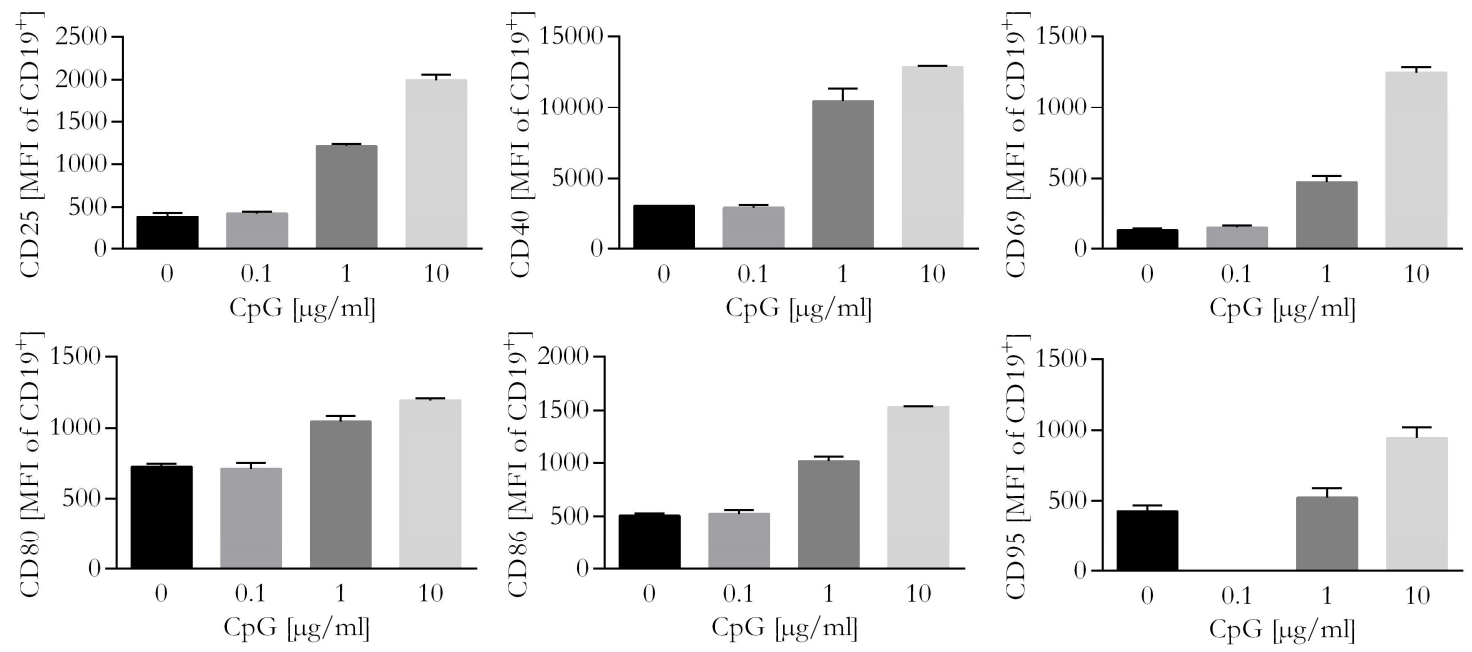

Figure 2: Cytosine-phosphate-guanine titration assay. Human peripheral blood mononuclear cells were stimulated in vitro for 20 hours with different concentrations of cytosine-phosphate-guanine (CpG). Flow cytometry was performed to measure the mean fluorescent intensity (MFI) of activation markers on B lymphocytes $\left(\mathrm{CD} 19^{+}\right)$. Data are presented as mean \pm standard error of the mean. $(n=3)$

The surface molecule CD150 is not constitutively expressed on myeloid-derived phagocytes, but upon activation of the cell. As myeloid cells express TLR-4, they can be activated by its ligand LPS. Hence, this stimulus was chosen and tested for the optimal concentration. As shown in Figure 3, a concentration of $100 \mathrm{pg} / \mathrm{ml}$ induced a significant upregulation of CD150 expression on $\mathrm{CD} 14^{+}$cells. For this reason, LPS stimulation was performed at a concentration of $100 \mathrm{pg} / \mathrm{ml}$ for all patient samples.

In a next step, it was examined whether in vitro LPS stimulation alters the composition of human PBMC. After 20 hours of stimulation, increasing concentrations of LPS induced a strong reduction of $\mathrm{CD} 14^{+}$cells and $\mathrm{CD} 4^{+} \mathrm{T}$ cells, while the other investigated cell types were compensatory increased (Figure 3). Thus, to avoid a methodical bias in this examination, cell population frequencies were assessed without any stimulation, providing a more accurate picture of the actual cell frequencies in the patients' blood.
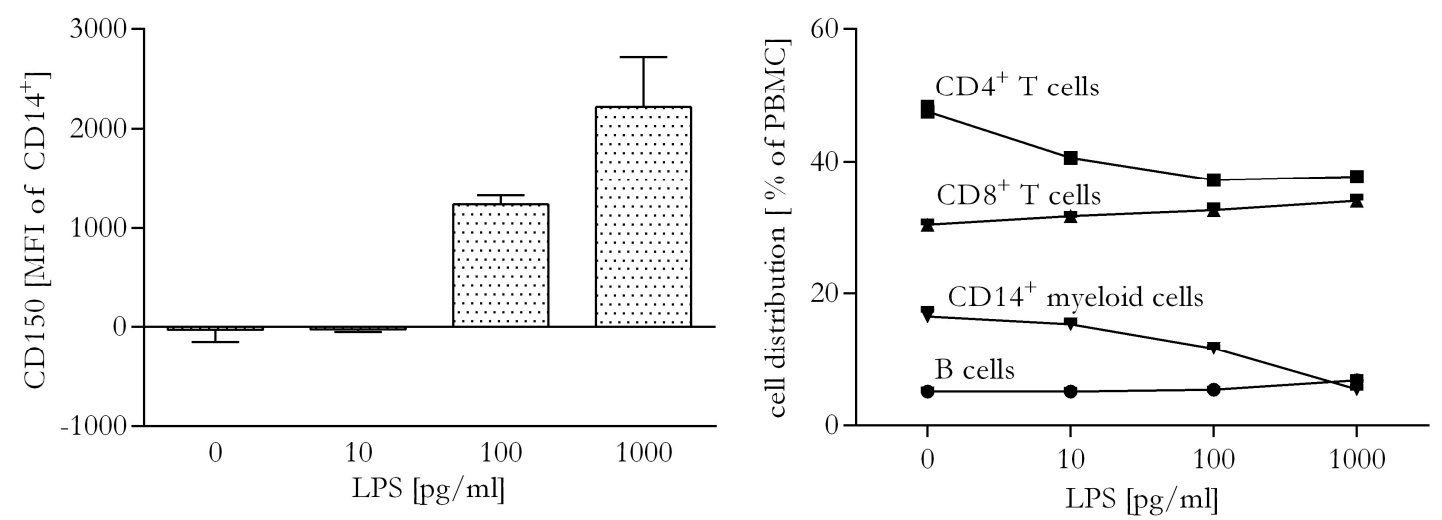

Figure 3: Lipopolysaccharides titration assay. Human peripheral blood mononuclear cells (PBMC) were stimulated in vitro for 20 hours with different concentrations of lipopolysaccharides (LPS). Flow cytometry was performed to measure the mean fluorescent intensity (MFI) of cluster of differentiation (CD)150 on myeloid phagocytes (CD14 ${ }^{+}$) (left graph). Cell frequencies of different cell types were also analyzed (right graph). Data are presented as mean \pm standard error of the mean $(n=3)$. 


\subsubsection{Intracellular staining}

To assess cytokine production by B cells ex vivo, flow cytometry analysis of intracellular antigens was performed. Therefore, cells were stimulated with CpG or LPS respectively and GolgiPlug was given to the cells to induce accumulation of cytokines within the Golgi apparatus, which makes cytokines easier detectable by this method. Next, cells were permeabilized to allow the intracellular antibodies enter the cell and bind their specific antigen. Flow cytometry then quantified the frequency of cytokine producing cells [\%] or the relative amount of accumulated cytokines [MFI] within the B cell gate.

To establish a valid and reliable strategy for the intracellular staining experiments, preexperiments had to be performed to adjust the individual parameters. In a first step, it was found that a pre-stimulation with $\mathrm{CpG}$ was necessary to make B cells produce cytokines at all. The optimal concentration was determined to be $1 \mu \mathrm{g} / \mathrm{ml}$ (data not shown). Further, an additional boost with ionomycin/PMA was necessary to increase the detectability of interleukins. However, the most relevant observation was that the duration of the antibody incubation had a huge impact on the detectability of the cytokines.

As presented in Figure 4, the prolongation of the incubation time with antibodies against the cytokines of interest from 30 minutes to 18 hours had a big effect on the measured cytokine expression. Especially the detection of IL-6 and TNF was relevantly enhanced. Thus, this experimental procedure was chosen for all patient samples, as an increased detection intensity (MFI) also increases the discriminatory power of the acquired data.
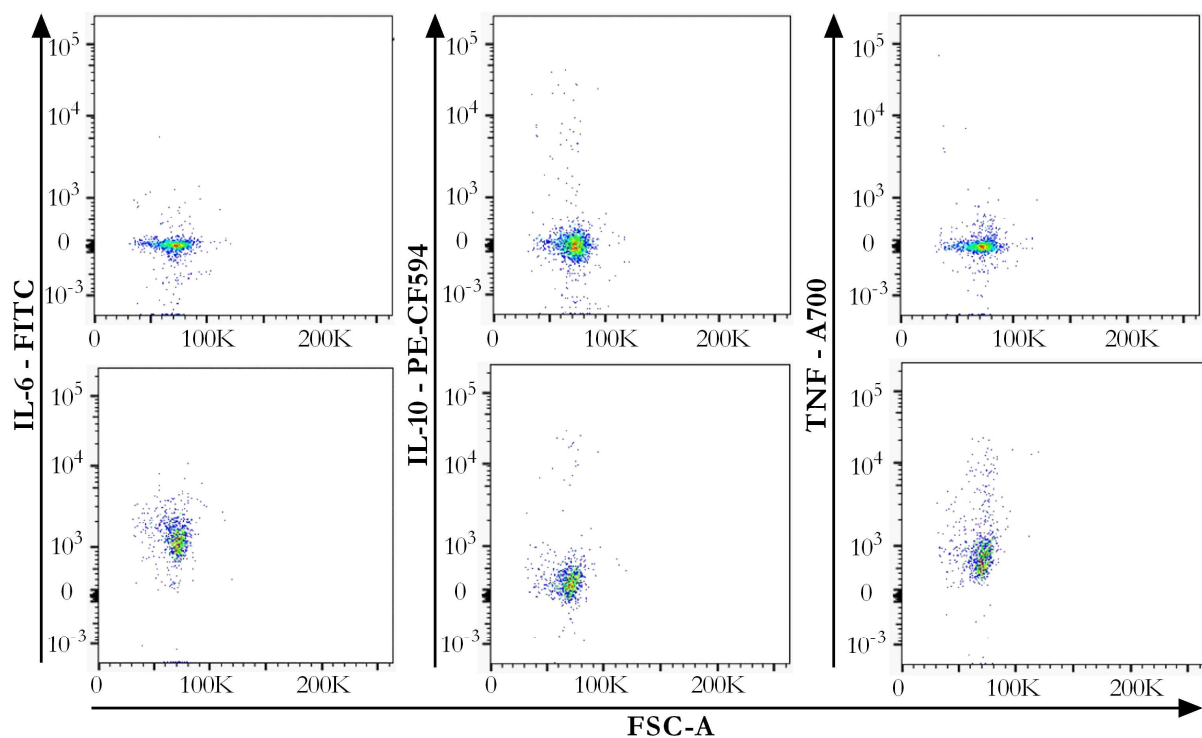

12 hours Fix/Perm $\downarrow$

+ intracellular staining $\downarrow$

$30 \mathrm{~min}$ incubation

$30 \mathrm{~min} \mathrm{Fix/Perm}$ $\downarrow$ + intracellular staining 16 hours incubation

Figure 4: Optimization of the intracellular staining. The flow-cytometric dot plots display the expression of the three cytokines of interest (interleukin-6 (IL-6); interleukin-10 (IL-10) and tumor necrosis factor (TNF)) on B cells (CD19+). In the upper row, intracellular stain incubation lasted only 30 minutes (min) after 12 hours of Fix/Perm application. The graphs below in represent the cytokine expression after 16 hours of incubation after only $30 \mathrm{~min}$ of Fix/Perm incubation (FSC $=$ forward scatter). 


\subsubsection{Compensation matrices}

The emission spectra of the used fluorophores have a natural overlap. To minimize the spillover of one fluorophore into the detection wavelength of another, the processing software is using so-called compensation matrices. By applying this mathematical method, a parallel detection of several fluorophore-conjugated antibodies in one panel is possible and reasonable. As not all patient samples could be measured in one run, several repeating measurement cycles had to be performed.

To find out which combinations of fluorophores are possible at all, different combinations were tested. Exemplarily, Figure 5 illustrates the effect of compensation on the flow cytometric dot plots. The reciprocal dependency of two antibodies with similar spectra could be removed completely in most cases, except their overlap was too large. This is due to the fact that two antibody-linked fluorophores with similar spectra have a high spectral overlap. Figure 5 shows that the closer the detection wavelengths of the antibodies are the more difficult it is to compensate them. This is the reason why in some cases, it is not possible to combine fluorophores.

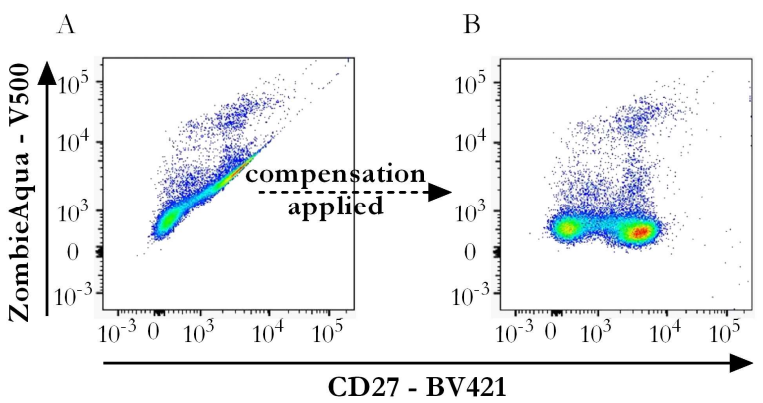

CD27 - BV421

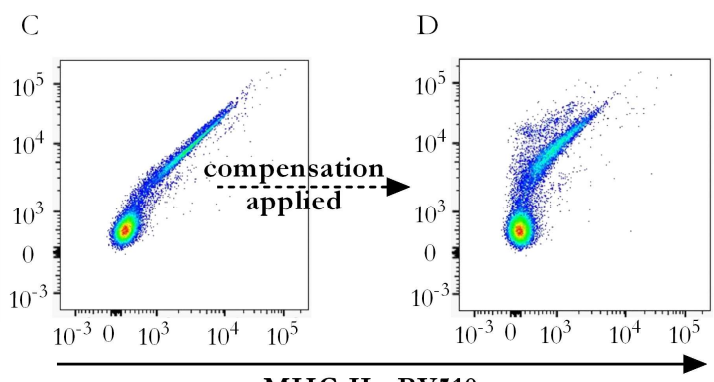

MHC-II - BV510

Figure 5: The effect of compensation. The dot plots show the intensity of the different fluorophores on cells excluded by size. These combinations had no biological relevance but helped in combining the different antibodies. $(\mathbf{A}+\mathbf{B})$ Shown is the effect of compensation between V500 (detected at 500nm wavelength) and BV421 (detected at 421 nm). As seen in the (B), the linear dependency between both fluorophores cannot be seen after compensation was applied. (C+D) In contrast, these dot plots represent detection wavelengths that are very close to each other (V500 (500nm) and BV510 (510nm)). In this case the compensation was not able to remove the reciprocal dependency $(\mathbf{D})$. 


\begin{tabular}{|c|c|c|c|c|c|c|c|c|c|}
\hline \multirow{10}{*}{$\begin{array}{c}\text { measurement } \\
\text { cycle } 1\end{array}$} & \multirow{9}{*}{$\begin{array}{l}\text { Show All } \\
\square \text { FITC.A :: CD } 14 \\
\square \text { PeICP.Cy5.5.A :: CD } 19 \\
\square \text { PE-CF594AA:: CD } 40 \\
\square \text { PE-Cy7.A :: CD80 } \\
\square \text { BV421.A :: CD86 } \\
\square \text { APC.A :: MHCII } \\
\square \text { PE-A :: FAS } \\
\square \text { APC-Cy7.A :: ZombieNIR }\end{array}$} & \multicolumn{6}{|c|}{ 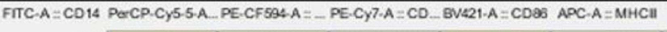 } & \multirow{2}{*}{$\begin{array}{r}P E-A=F A S \\
1.0127\end{array}$} & \multirow{2}{*}{$\begin{array}{r}\text { APC.C. } 7 \cdot A=Z \\
0.3098\end{array}$} \\
\hline & & 100 & 3.2765 & 1.381 & 0.5524 & 3.785 & 1.0701 & & \\
\hline & & 0 & 100 & 0 & 7.660 & 0 & 11,2786 & 0 & 1.2607 \\
\hline & & 1,3156 & 16.3741 & 100 & 3.3045 & 3.633 & 0.7211 & 17,4088 & 0.3143 \\
\hline & & 5.8511 & 5.8511 & 4.443 & 100 & 13.6823 & 3.4686 & 5.2387 & 1.8864 \\
\hline & & 1.9723 & 1.289 & 1.3025 & 0.2112 & 100 & 0.4361 & 1.3025 & 0 \\
\hline & & 0.274 & 0.9324 & 0.5485 & 2.0085 & 0.7272 & 100 & 0.2339 & 1.2657 \\
\hline & & 0,7487 & 6.5921 & 82,2149 & 0.886 & 0.2458 & 0.0728 & 100 & 0.0121 \\
\hline & & 0.2953 & 37.6249 & 0.2785 & 89,1972 & 0 & 5.1131 & 0.1792 & 100 \\
\hline & $\square$ Show All & \multicolumn{6}{|c|}{ 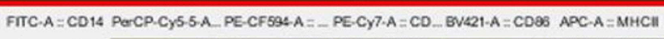 } & $P E \cdot A=F A S$ & $A P C \cdot C Y 7 \cdot A=Z$ \\
\hline \multirow{8}{*}{$\begin{array}{c}\text { measurement } \\
\text { cycle } 2\end{array}$} & \multirow{8}{*}{$\begin{array}{l}\square \text { FITC.A :: CD } 14 \\
\square \text { PeICP.CY5.5.A :: CD } 19 \\
\square \text { PE-CF594A :: CD40 } \\
\square \text { PE-Cy7-A :: CD80 } \\
\square \text { BV421.A :: CD86 } \\
\square \text { APC.A :: MHCII } \\
\square \text { PE-A :: FAS } \\
\square \text { APC.CY7.A :: ZombieNIR }\end{array}$} & 100 & 4.4073 & 1.5291 & 0.8583 & 4.9285 & 1.0477 & 1,284 & \begin{tabular}{|l|} 
\\
0.3742 \\
\end{tabular} \\
\hline & & 0 & 100 & 0.2329 & 8.8075 & 0 & 11,3701 & 0 & 1.252 \\
\hline & & 1,4515 & 16,4133 & 100 & 3.385 & 3,0285 & 0,3193 & 16,7985 & 0 \\
\hline & & 10.0772 & 6,7448 & 5,4858 & 100 & 17.8354 & 3,9811 & 5,9353 & 1,2378 \\
\hline & & 3.1573 & 1,6982 & 1,5807 & 0,3807 & 100 & 0.4541 & 1,1039 & 0 \\
\hline & & 0,4436 & 1.0351 & 0.6999 & 1,9027 & 0.8521 & 100 & 0.2485 & 1,3027 \\
\hline & & 0.8315 & 6.4078 & 62,1024 & 0.8454 & 0,3993 & 0 & 100 & 0 \\
\hline & & 0.7194 & 42,8358 & 0,319 & 81,1535 & 0,1741 & 5.1998 & 0.1975 & 100 \\
\hline \multirow{9}{*}{$\begin{array}{c}\text { measurement } \\
\text { cycle } 3\end{array}$} & \multirow{9}{*}{$\begin{array}{l}\square \text { FITC.A :: CD } 14 \\
\square \text { PeICP.CY5.5.A :: CD } 19 \\
\square \text { PE-CF5944A :: CD40 } \\
\square \text { PE-Cy7-A :: CD80 } \\
\square \text { BV421.A :: CD88 } \\
\square \text { APC.A :: MHCII } \\
\square \text { PE.A :: FAS } \\
\square \text { APC.Cy7.A :: ZombieNIR }\end{array}$} & $\mathrm{FITC} \cdot \mathrm{A}=\mathrm{CD} 14$ & CP.CY5.5.A. & $C F 594 A=-8$ & $\mathrm{Cy} 7 \mathrm{~A}=\mathrm{CD}-\mathrm{B}$ & $21 \cdot A=\operatorname{CD} 86$ & $\overline{D C-A=M H C I I}$ & $P E A=F A S$ & $A D C \cdot C y 7 \cdot A=Z$ \\
\hline & & 100 & 4.098 & 2.0401 & 0.3472 & 4.8411 & 0.9958 & 1,5626 & 0 \\
\hline & & 0 & 100 & 0 & 7.2406 & 0 & 11.0303 & 0 & 1,2747 \\
\hline & & 1.422 & 17.0928 & 100 & 3.6301 & 2.8292 & 0,3884 & 17,6949 & 0.1494 \\
\hline & & 10.2022 & 8.6857 & 8.75 & 100 & 15.1029 & 4.4449 & 7.5 & 3.2978 \\
\hline & & 3.1294 & 2.036 & 2.2647 & 0.3846 & 100 & 0.9411 & 1.7092 & 0.1568 \\
\hline & & 0.4315 & 0.9827 & 0.5328 & 1.8885 & 0.7966 & 100 & 0.2421 & 1.1242 \\
\hline & & 0.8709 & 0.4402 & 61.8343 & 0.8752 & 0,3981 & 0.1196 & 100 & 0.0513 \\
\hline & & 0.0672 & 39.6898 & 0.093 & 80.7033 & 0 & 5.5254 & 0.0592 & 100 \\
\hline
\end{tabular}

Figure 6: Compensation matrices of the antigen presentation panel (e). The percentages in the boxes indicate the spectral overlap between the different fluorophores. Exemplarily, three measurement cycles of panel (e) are displayed. The red marked compensation matrix was chosen and applied for all samples in panel (e). The colors of the single values indicate the extend of the spectral overlaps.

To determine whether one general compensation matrix should be applied to all samples of one panel or whether each run should be compensated separately, the obtained spectral overlaps of the different measurement cycles were compared with each other. An example of different compensations matrices acquired in separate runs is displayed in Figure 6. It was observed that the overlap values between the distinct fluorophores differ only slightly when different measurement cycles were compared to each other. Therefore, one compensation matrix was applied to all runs (Figure 6, red frame) to avoid data processing bias. This procedure was carried out for each panel separately (data not shown). Being in line with the example in Figure 6, they differed only marginally from each other and one compensation matrix was chosen for all runs of each panel.

This aspect was very important when staining panels were created as these findings had to be taken into consideration to prevent dependency of one marker of another. Therefore, several combinations were tested before the final five panels as displayed in Figure 7 were created and applied. 


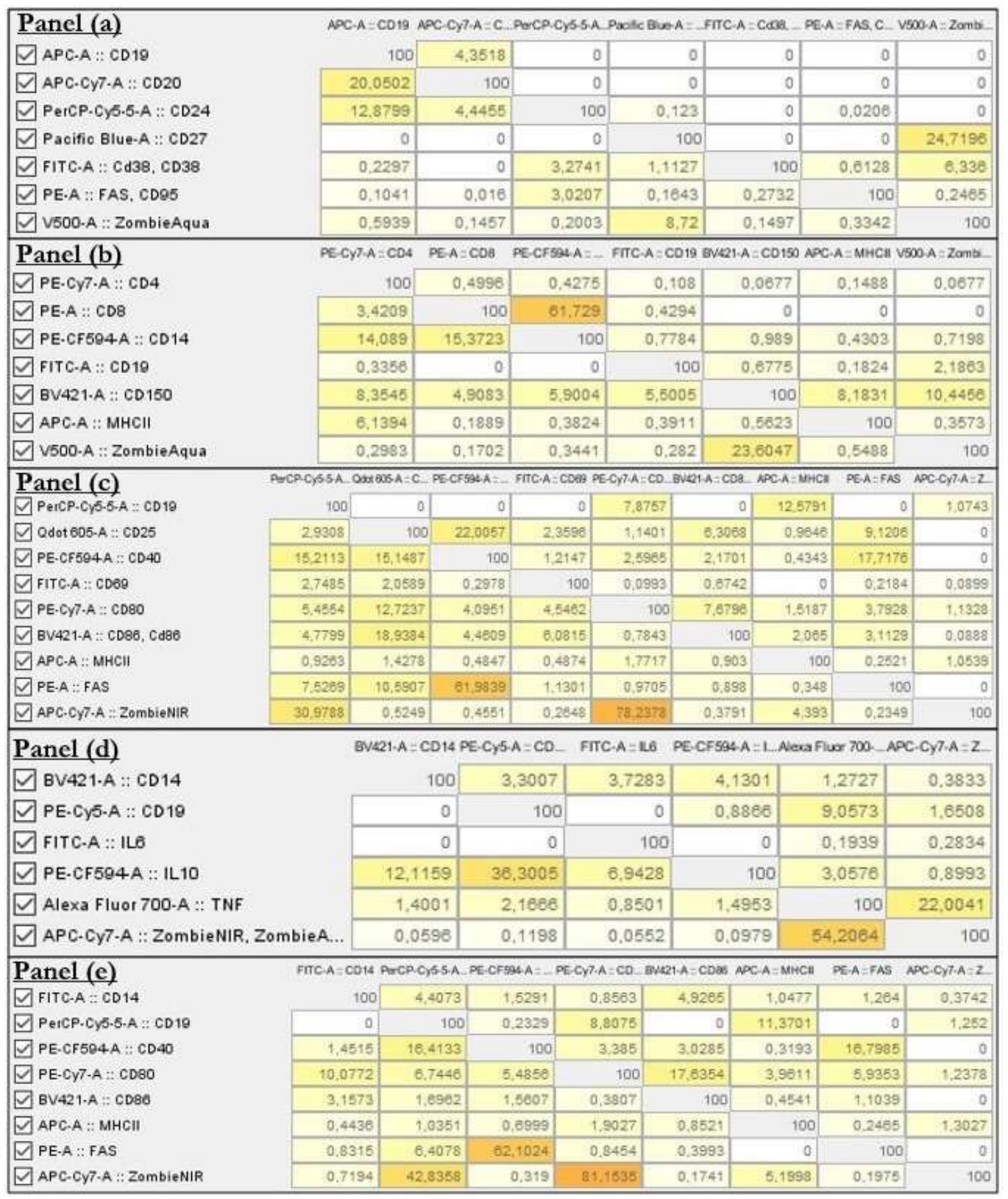

Figure 7: Compensation matrices of all five acquisition panels. The letters indicate the respective panel as described above. Spectral overlap of the single fluorophores is given in percent; colors identify high values.

\subsection{Cell distribution and survival}

DMF is thought to act as an immunomodulatory drug. To find out, what kind of cell types are affected by DMF treatment, the general composition of human PBMC was examined. This included the frequency of myeloid-derived phagocytes $\left(\mathrm{CD} 14^{+}\right), \mathrm{T}_{\mathrm{h}}$ cells $\left(\mathrm{CD} 4^{+}\right)$, cytotoxic T cells $\left(\mathrm{CD} 8^{+}\right)$and $\mathrm{B}$ cells $\left(\mathrm{CD} 19^{+}\right)$, as they represent the four major immune cell types within the PBMC. Prior to that, it was analyzed whether DMF alters the survival of immune cells in cell culture. 


\subsubsection{DMF treatment decreased the survival of PBMC in culture}

As previously shown in Figure 1A, dead and ruptured cells were excluded by their size (necrosis) and the live/dead indicator dye Zombie, which stains apoptotic cells. This step is required, since in the process of cell freezing, thawing, preparation and incubation, cells experience physical and chemical stress leading to their necrosis and/or apoptosis. Small, ruptured and dead cells were excluded from the analysis, as their antigen expression can be strongly altered compared to living cells and does not represent the physiological condition in the patients' blood. While small cells are a hint for dead cells that lose their integrity, Zombie dye positive cells mainly represent apoptotic cells. It was found that DMF therapy changed the resistance of PBMC against this external stress with a reduced proportion of intact cells in the DMF-treated samples (Figure 8). This significant finding was seen in all stimulation regimes, resembling a relative increase of necrotic cells upon DMF treatment.
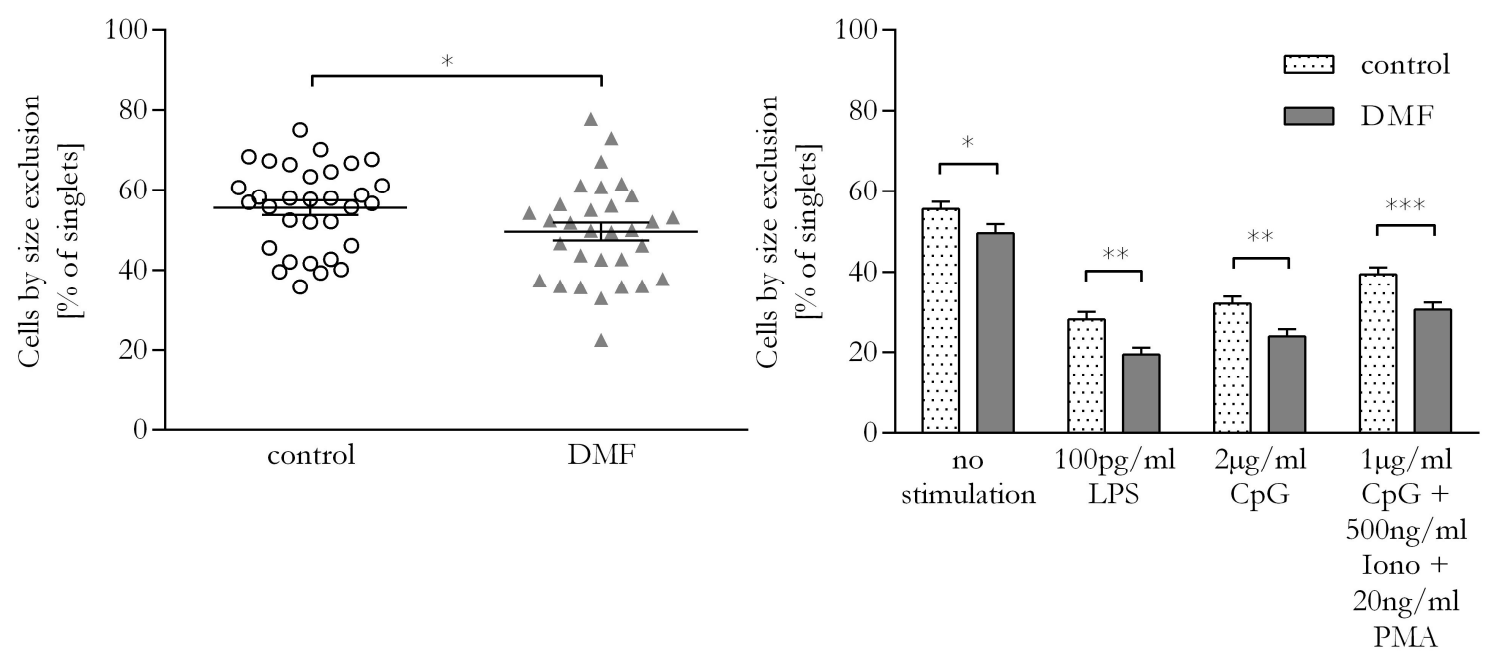

Figure 8: Dimethyl fumarate decreased the proportion of intact cells (excluded by size). Intact shaped cells were found using size exclusion in the forward scatter (FSC) / side scatter (SSC) dot plot. The left graph shows the frequency of intact cells within the pool of singlets without any stimulation. Control multiple sclerosis patients (circles) were compared to dimethyl fumarate (DMF)-treated matches (triangles). On the right side, mean intact cell frequencies of the different stimulation regimes are plotted. Bars indicate mean \pm standard error of the mean. ( $\mathrm{CpG}=$ cytosine-phosphate-guanine; Iono = ionomycin; PMA = 12-O-Tetradecanoylphorbol-13-acetate; LPS = lipopolysaccharide; $\mathrm{n}=31$ (DMF); $\mathrm{n}=30$ (control); unpaired t-test; $*=\mathrm{p}<0.05 ; * *=\mathrm{p}<0.01 ; * * *=\mathrm{p}<0.001$ )

Regarding Zombie negative, living cells, this effect was not as strong. Figure 9 shows that DMF treatment reduced the frequency of living cells within all size-excluded cells only upon the $\mathrm{CpG}+$ ionomycin + PMA stimulated condition. This suggests that apoptosis was only increased after strong stimulation. 

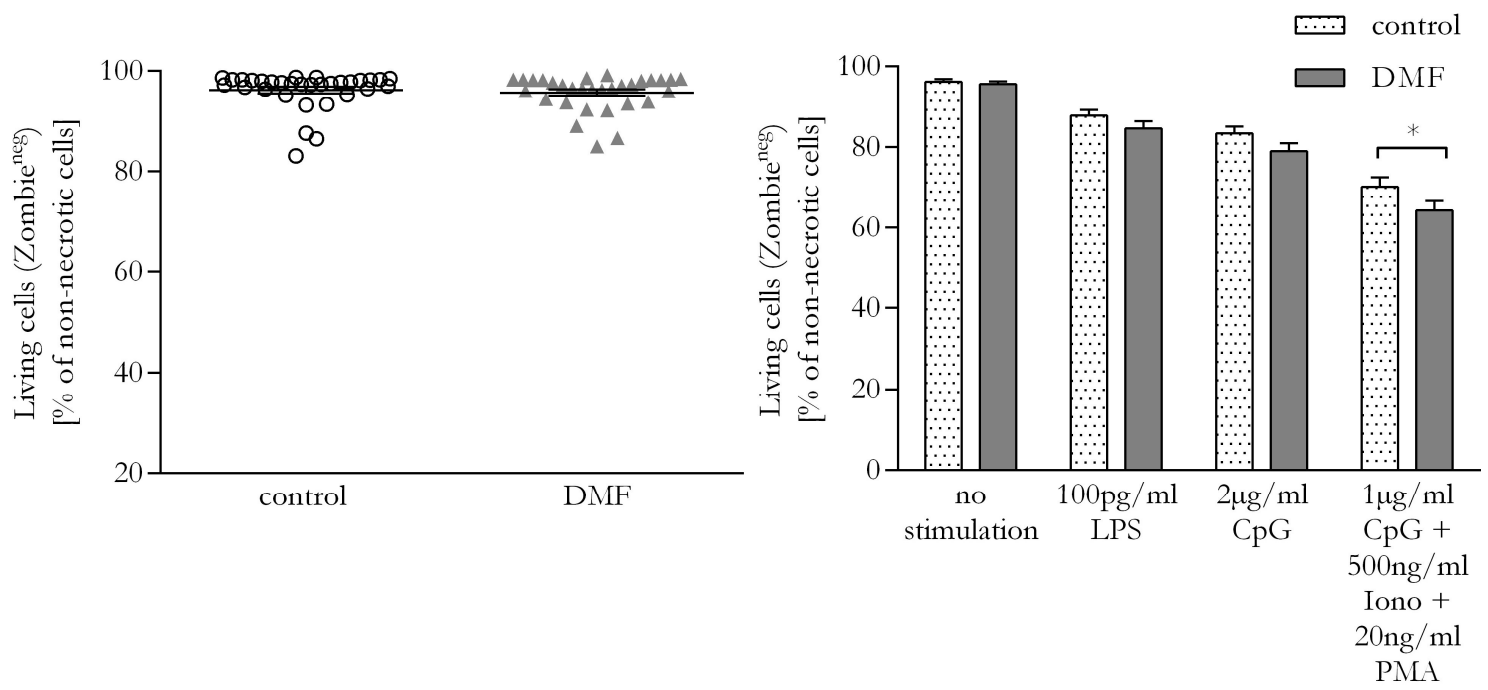

Figure 9: Upon stimulation, the proportion of living cells was reduced by dimethyl fumarate. Living cells were defined as Zombie negative. The left graph shows the frequency of living cells within the pool of intact cells (size excluded) without any stimulation. Control multiple sclerosis patients (circles) were compared to dimethyl fumarate (DMF)-treated matches (triangles). On the right side, mean living cell frequencies of the different stimulation regimes are plotted. Bars indicate mean \pm standard error of the mean. $(\mathrm{CpG}=$ cytosine-phosphate-guanine; Iono $=$ ionomycin; PMA $=$ 12-O-Tetradecanoylphorbol-13-acetate; LPS = lipopolysaccharide; $n=31$ (DMF); $n=30$ (control); unpaired t-test; * = $\mathrm{p}<0.05)$

To identify if the observed DMF-induced vulnerability towards external stress is more prominent in certain cell types, subpopulations were analyzed for apoptosis using Zombie dye. DMF treatment mainly affected the survival of B cells but did not change the frequency of living myeloid-derived phagocytes (Figure 10).
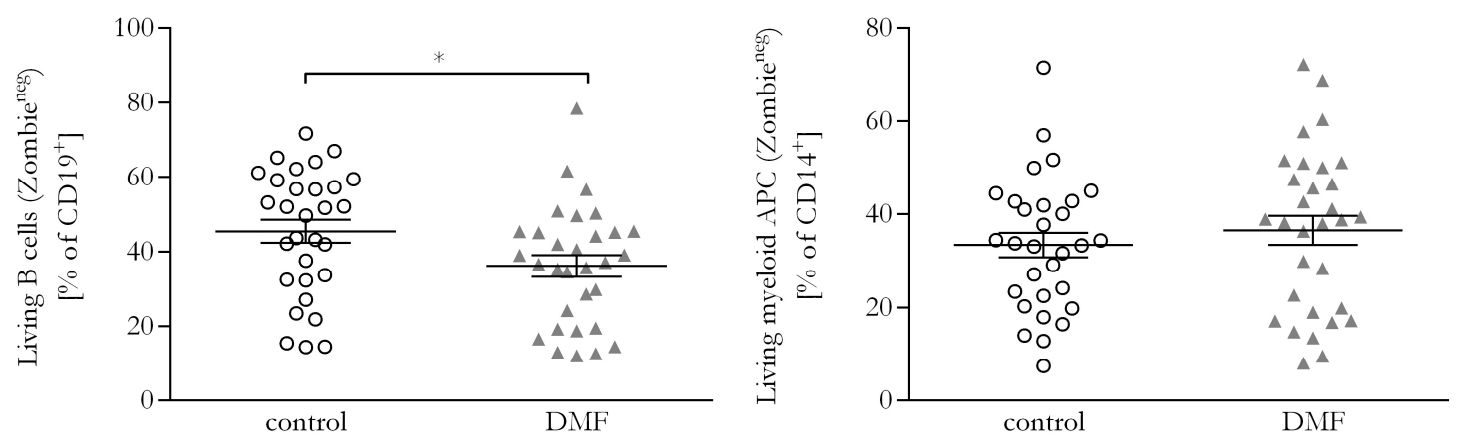

Figure 10: Dimethyl fumarate decreased the frequency of living B cells. Living cells were defined as Zombie negative. In this case, not stimulated B cells $\left(\mathrm{CD} 19^{+}\right)$and myeloid-derived phagocytes $\left(\mathrm{CD} 14^{+}\right)$were gated directly after size exclusion. Next, the Zombie dye signal was used to identify living cells. Control multiple sclerosis patients (circles) were compared to dimethyl fumarate (DMF)-treated matches (triangles). Bars indicate mean \pm standard error of the mean. (APC $=$ antigen-presenting cells; $n=31$ (DMF); $n=30$ (control); unpaired t-test; $*=\mathrm{p}<0.05$ )

\subsubsection{DMF treatment leads to an increased $\mathrm{CD}^{+} 4^{+}$myeloid cell frequency within the PBMC}

Next, it was evaluated how DMF changes the frequency of the distinct cell populations. As shown in Figure 11, the relative proportion of myeloid-derived phagocytes (being defined 
as $\mathrm{CD} 14^{+}$) within the PBMC pool was significantly increased upon DMF treatment in the cross-sectional analysis of all blood samples. A stimulation of the samples with $2 \mu \mathrm{g} / \mathrm{ml} \mathrm{CpG}$ or $100 \mathrm{pg} / \mathrm{ml}$ LPS for 20 hours caused a lower CD14 ${ }^{+}$myeloid cell frequency when compared to the unstimulated measurements. However, the trend towards an increased $\mathrm{CD} 14^{+}$myeloid cell frequency could also be seen in those experiments.
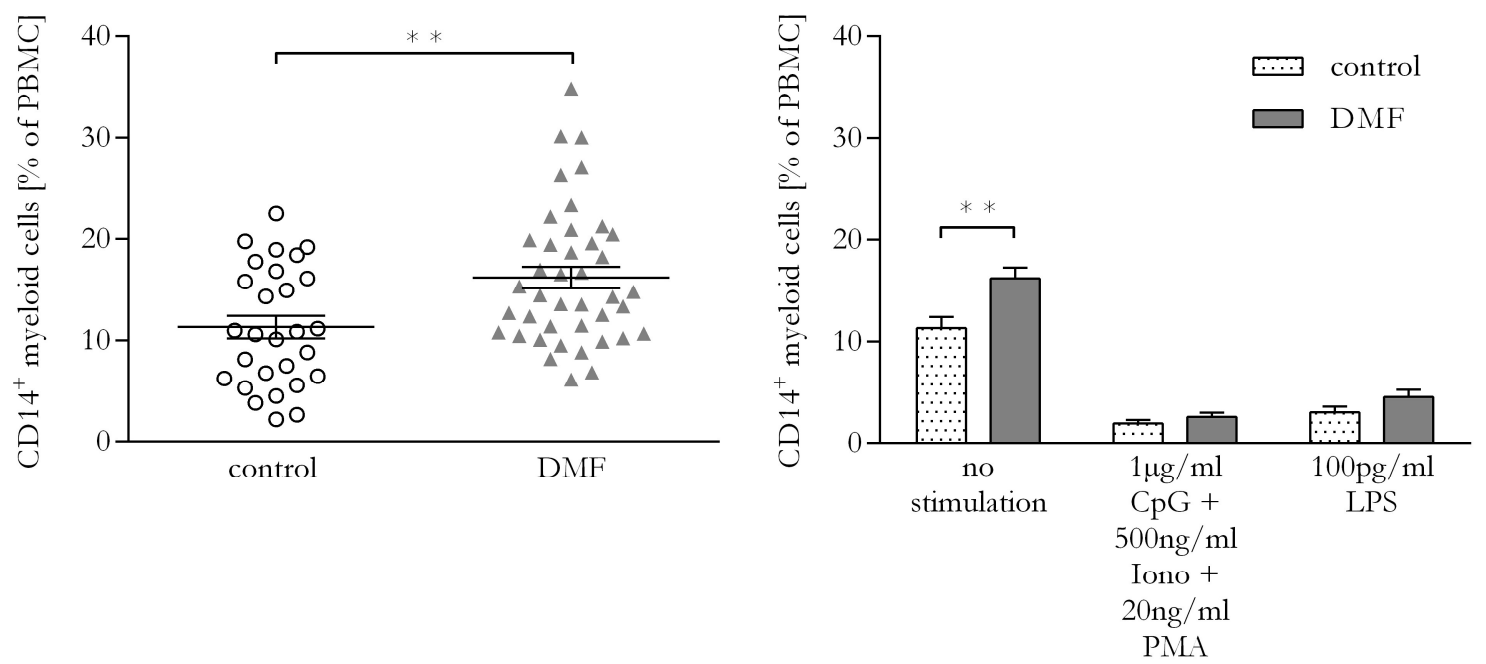

Figure 11: $\mathrm{CD} 14^{+}$myeloid cell frequency increased upon dimethyl fumarate treatment. The left graph shows the frequency of myeloid-derived phagocytes $\left(\mathrm{CD}^{+}\right)$within the peripheral blood mononuclear cell (PBMC) pool without any stimulation. Control multiple sclerosis patients (circles) were compared to dimethyl fumarate (DMF)-treated matches (triangles). On the right side, mean $\mathrm{CD}_{1} 4^{+}$myeloid cell frequencies of the different stimulation regimes are plotted. Bars indicate mean \pm standard error of the mean. ( $\mathrm{CpG}=$ cytosine-phosphate-guanine; Iono = ionomycin; PMA = 12-O-Tetradecanoylphorbol-13-acetate; LPS = lipopolysaccharide; $n=31$ (DMF); $n=30$ (control); unpaired t-test; ** = $\mathrm{p}<0.01)$

\subsubsection{No change in the frequency of $\mathrm{CD}^{+}$but relative reduction of $\mathrm{CD}^{+} \mathrm{T}$ cells}

To determine whether DMF treatment has an influence on the distribution of $\mathrm{T}_{\mathrm{h}}$ cells versus cytotoxic $\mathrm{T}$ cells, subpopulations were analyzed separately. This investigation revealed that DMF treatment did not alter the proportion of $\mathrm{CD}^{+} \mathrm{T}$ cells $\left(\mathrm{T}_{\mathrm{h}}\right.$ cells) within all PBMC in both the unstimulated and the LPS-stimulated condition (Figure 12). 

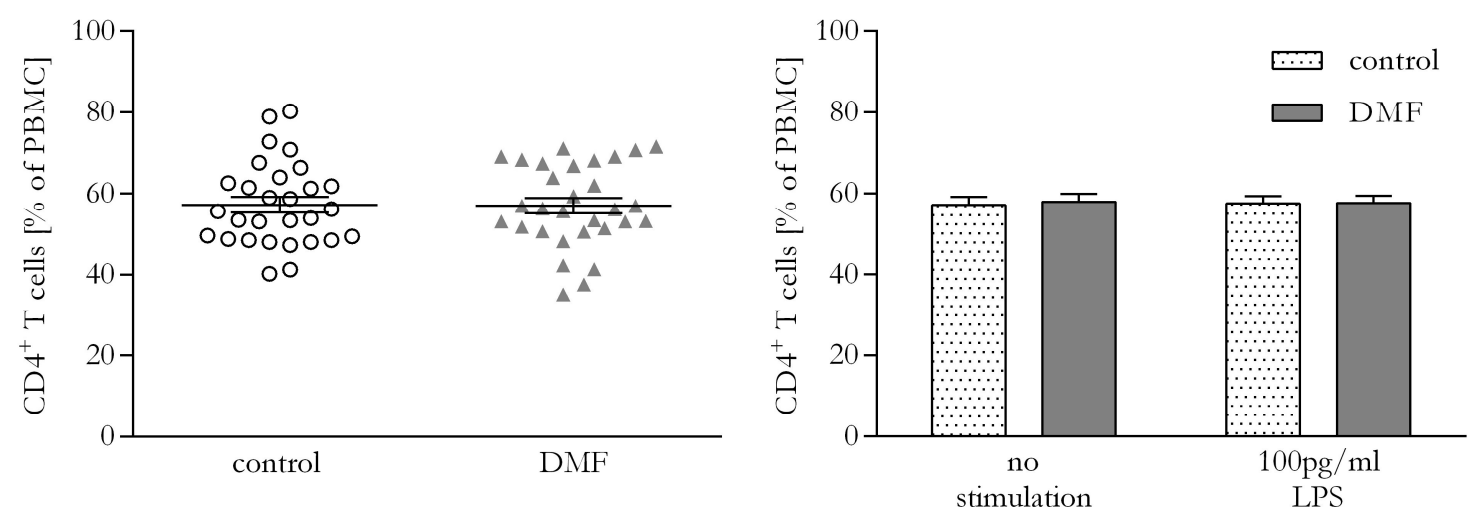

Figure 12: $\mathrm{CD}^{+} \mathrm{T}$ cell frequency was not altered by dimethyl fumarate. The left graph shows the frequency of $\mathrm{CD} 4^{+} \mathrm{T}$ cells within the peripheral blood mononuclear cell (PBMC) pool without any stimulation. Control multiple sclerosis patients (circles) were compared to dimethyl fumarate (DMF)-treated matches (triangles). On the right side, mean $\mathrm{CD} 4{ }^{+} \mathrm{T}$ cell frequencies of the different stimulation regimes are plotted. Bars indicate mean \pm standard error of the mean. (LPS = lipopolysaccharide; $n=31$ (DMF); $n=30$ (control); unpaired t-test; not significant)

The frequency of cytotoxic $\mathrm{T}$ cells, defined as $\mathrm{CD} 8^{+}$, was significantly decreased upon DMF treatment when cells were stimulated with $100 \mathrm{pg} / \mathrm{ml}$ LPS. A similar trend could be observed in the non-stimulated measurements (Figure 13).
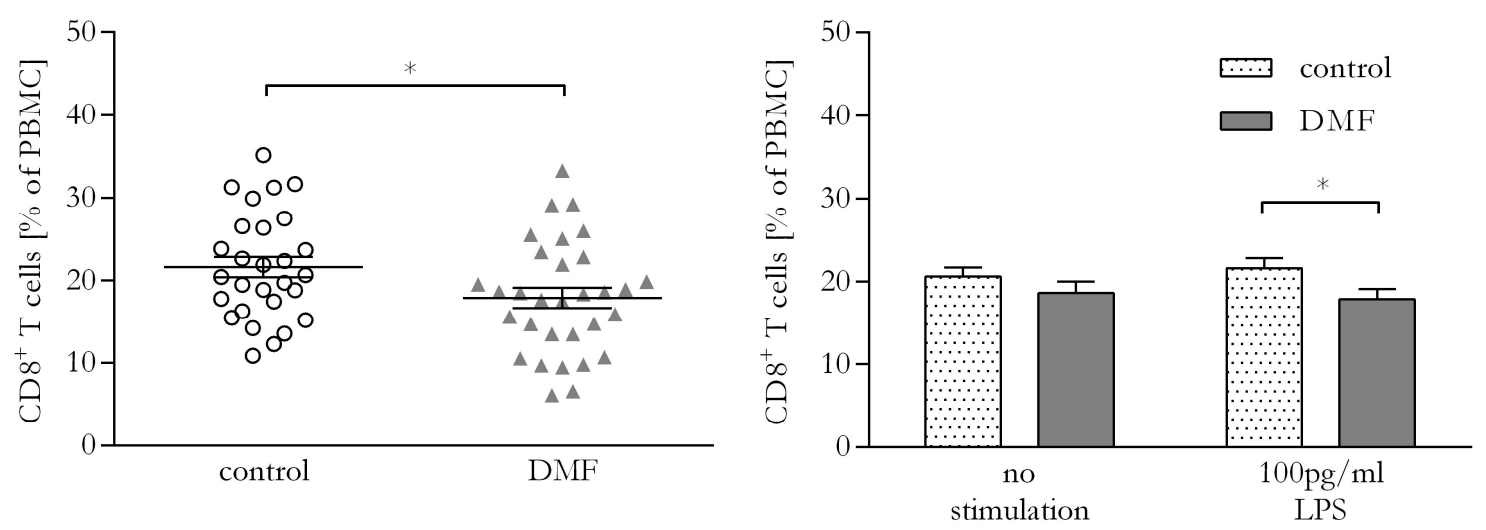

Figure 13: Dimethyl fumarate treatment reduced the frequency of $\mathrm{CD} 8^{+} \mathrm{T}$ cells. The left graph shows the frequency of $\mathrm{CD}^{+} \mathrm{T}$ cells within the peripheral blood mononuclear cell (PBMC) pool after an in vitro stimulation with $100 \mathrm{pg} / \mathrm{ml}$ lipopolysaccharide (LPS). Control multiple sclerosis patients (circles) were compared to dimethyl fumarate (DMF)-treated matches (triangles). On the right side, mean $\mathrm{CD} 8^{+} \mathrm{T}$ cell frequencies of the different stimulation regimes are plotted. Bars indicate mean \pm standard error of the mean. ( $n=31$ (DMF); $n=30$ (control); unpaired t-test; not significant)

\subsubsection{B cell frequency was not changed upon DMF treatment}

Since B cells were of major interest in this study, their frequency within all PBMC was obtained in every panel. In all different stimulation regimes, there was no significant change when DMF-treated patients were compared to controls with a slight, not significant tendency towards an increase (Figure 14). 

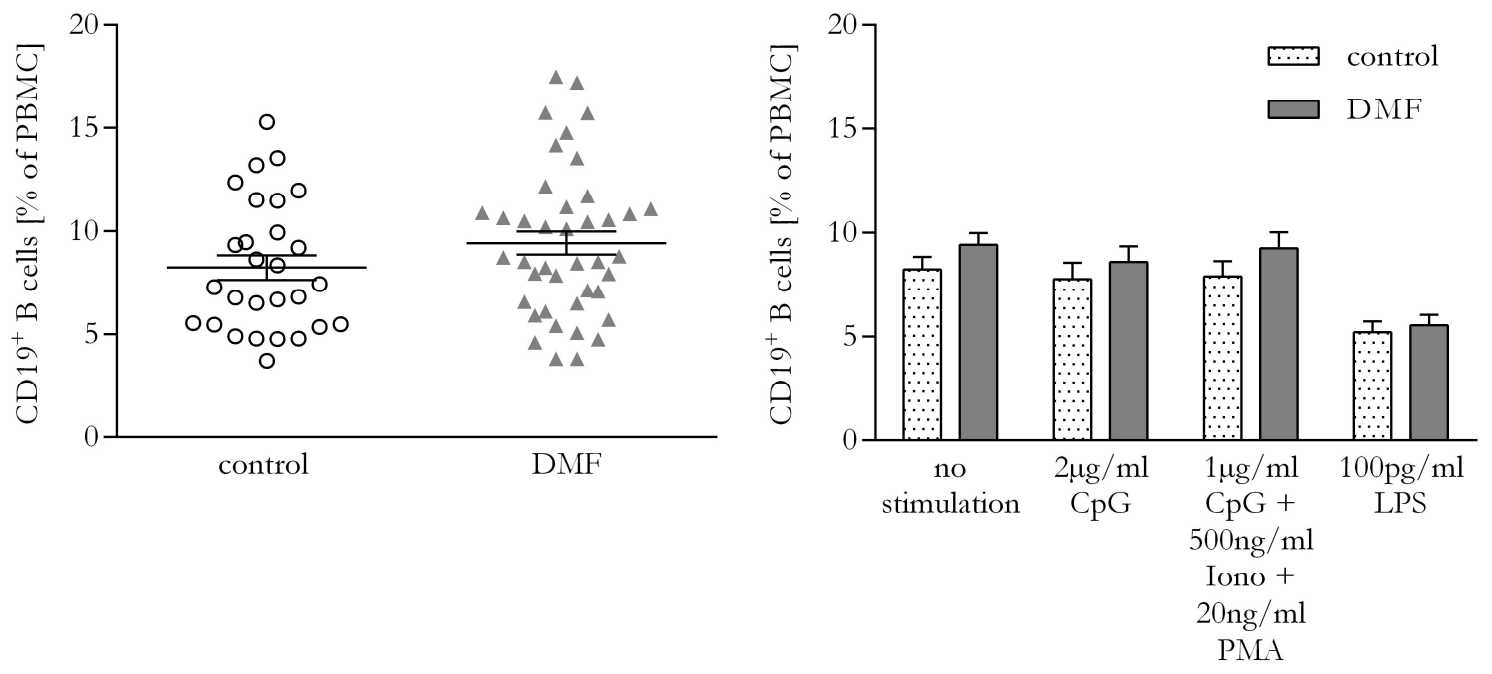

Figure 14: B cell frequency was not altered by dimethyl fumarate. The left graph shows the frequency of B cells $\left(\mathrm{CD} 19^{+}\right)$ within the peripheral blood mononuclear cell (PBMC) pool without any stimulation. Control multiple sclerosis patients (circles) were compared to dimethyl fumarate (DMF)-treated matches (triangles). On the right side, mean B cell frequencies of the different stimulation regimes are plotted. Bars indicate mean \pm standard error of the mean. (CpG $=$ cytosinephosphate-guanine; Iono = ionomycin; PMA = 12-O-Tetradecanoylphorbol-13-acetate; LPS = lipopolysaccharide; $n=31$ (DMF); $n=30$ (control); unpaired t-test; not significant)

Table 12: Summary of cell frequency changes. ( $\mathrm{SD}=$ standard deviation; $\mathrm{DMF}=$ dimethyl fumarate; $\mathrm{CD}=$ cluster of differentiation; $\mathrm{PBMC}=$ peripheral blood mononuclear cells; ns = not significant)

\begin{tabular}{|l|c|c|c|c|c|}
\hline & $\begin{array}{c}\text { Control } \\
\text { (mean } \pm \text { SD) }\end{array}$ & $\begin{array}{c}\text { DMF-treated } \\
\text { (mean } \pm \text { SD) }\end{array}$ & $\begin{array}{c}\text { Percental } \\
\text { change }\end{array}$ & p value & \\
\hline CD14+ (\% of PBMC) & $11.31 \pm 1.11$ & $16.21 \pm 1.06$ & $+43.3 \%$ & 0.0028 & $* *$ \\
$\mathbf{C D} 4+(\%$ of PBMC) & $57.21 \pm 1.91$ & $57.02 \pm 1.87$ & $-0.3 \%$ & 0.9353 & $\mathrm{~ns}$ \\
$\mathbf{C D}^{+}(\%$ of PBMC) & $21.59 \pm 1.22$ & $17.88 \pm 1.22$ & $-17.2 \%$ & 0.0359 & $*$ \\
$\mathbf{C D} 19^{+}(\%$ of PBMC) & $8.25 \pm 0.60$ & $9.43 \pm 0.56$ & $+14.3 \%$ & 0.1635 & $\mathrm{~ns}$ \\
\hline
\end{tabular}

\subsection{B cell subsets}

Next, it was tested whether DMF has a differential effect on B cell subsets and/or maturation stages, even though the total B cell frequency is not altered. Therefore, based on their distinct expression of surface markers, the frequencies of transitional B cells (CD24 $\left.4^{\text {high }} \mathrm{CD} 38^{\text {high }}\right)$, mature B cells $\left(\mathrm{CD} 24^{\text {high }} \mathrm{CD} 38^{\text {low }}\right)$, antigen-experienced B cells $\left(\mathrm{CD} 27^{+}\right)$, memory B cells $\left(\mathrm{CD} 27^{\text {var }} \mathrm{CD} 38^{\text {low }}\right)$ and plasmablasts $\left(\mathrm{CD} 20^{-} \mathrm{CD} 27^{+} \mathrm{CD} 38^{+}\right)$were determined.

\subsubsection{DMF increased the immature, transitional B cell frequency}

After the generation of B cells in the bone marrow, their earliest stage released in blood are immature, transitional B cells characterized by their high expression of CD24 and CD38. As shown in Figure 15, the frequency of these immature B cells within all PBMC was 
significantly increased upon DMF treatment. Complementary, the proportion of mature B cells defined as $\mathrm{CD} 24^{\mathrm{var}}$ and $\mathrm{CD} 38^{\text {low }}$ was reduced upon DMF treatment (Figure 15).
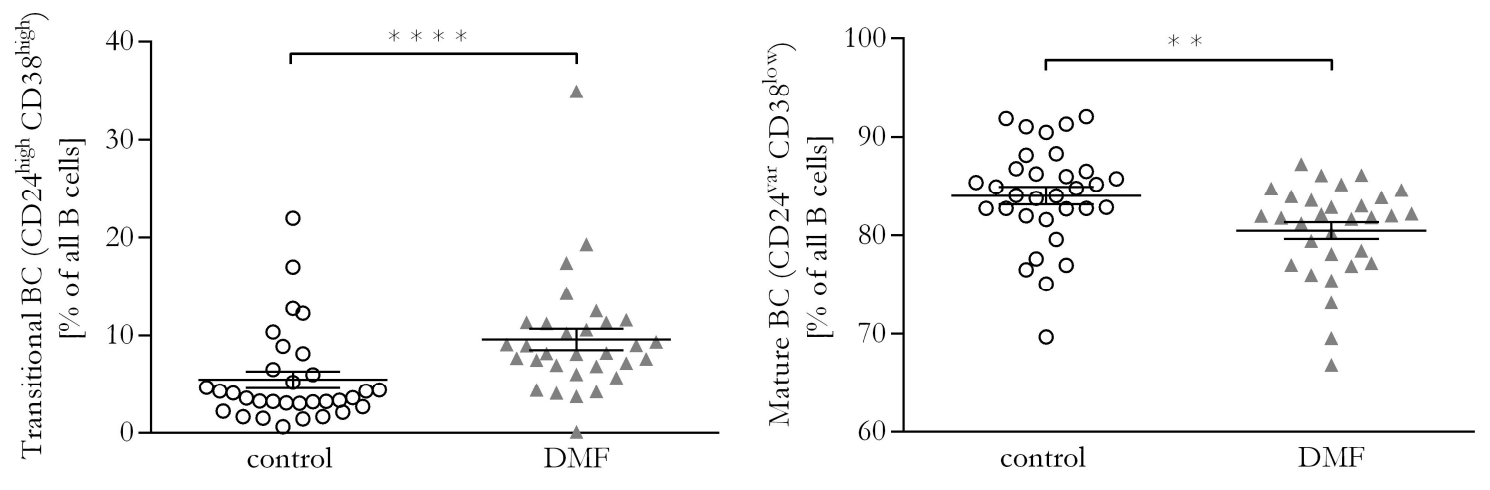

Figure 15: Dimethyl fumarate treatment increased transitional B cell frequency. Transitional B cell (BC) (CD24high $\left.\mathrm{CD} 38^{\text {high }}\right)$ and mature $\mathrm{BC}\left(\mathrm{CD} 24^{\text {var }} \mathrm{CD} 38^{\text {low }}\right)$ frequency of not stimulated pre-gated $\mathrm{BC}\left(\mathrm{CD} 19^{+}\right)$was measured using flow cytometry. Control multiple sclerosis patients (circles) were compared to dimethyl fumarate (DMF)-treated matches (triangles). Bars indicate mean \pm standard error of the mean. $(n=31$ (DMF); $n=30$ (control); unpaired t-test; ** $=\mathrm{p}<0.01$; $* * * *=\mathrm{p}<0.0001)$

\subsubsection{The frequency of antigen-experienced B cells was reduced in DMF-treated patients}

Antigen-experienced B cells can be characterized by their high expression of CD27. As presented in Figure 16, there was a significant reduction of these cells in the B cell pool of DMF-treated patients when compared to controls with an absolute reduction by $11.7 \%$.
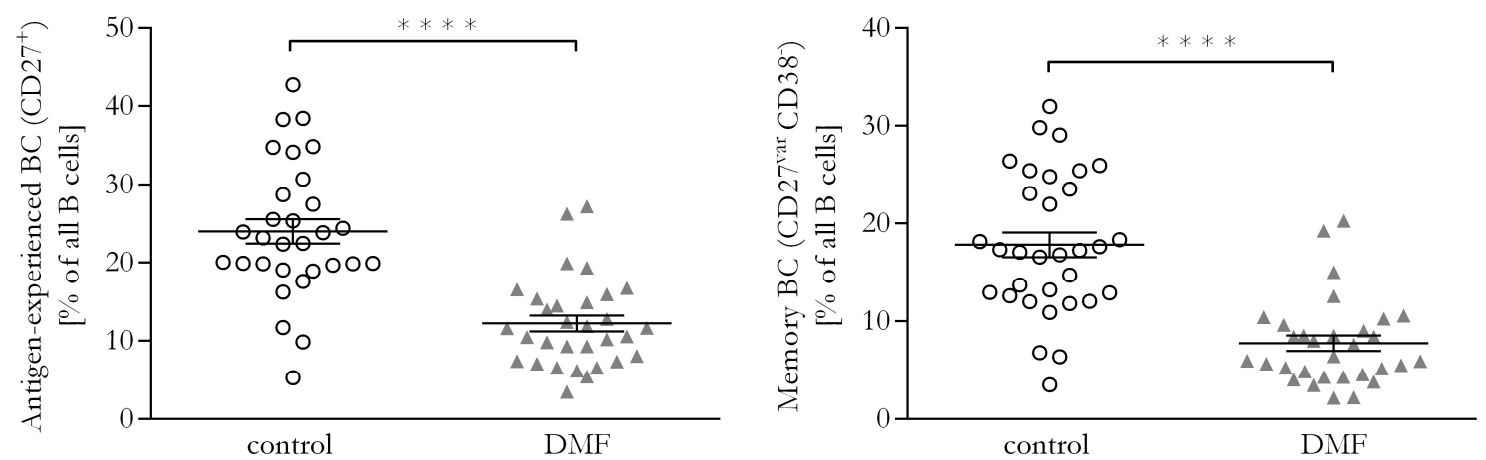

Figure 16: Antigen-experienced and memory $\mathrm{B}$ cell frequencies were reduced by dimethyl fumarate. Antigen-experienced $\mathrm{B}$ cell $(\mathrm{BC})\left(\mathrm{CD} 27^{+}\right)$and memory $\mathrm{BC}\left(\mathrm{CD} 27^{\mathrm{var}} \mathrm{CD} 38-\right)$ frequency of not stimulated pre-gated $\mathrm{BC}\left(\mathrm{CD} 19^{+}\right)$was measured using flow cytometry. Control multiple sclerosis patients (circles) were compared to dimethyl fumarate (DMF)-treated matches (triangles). Bars indicate mean \pm standard error of the mean. ( $n=31$ (DMF); $n=30$ (control); unpaired t-test; **** $=\mathrm{p}<0.0001)$

\subsubsection{DMF specifically reduced memory $B$ cell frequencies in MS patients}

Another investigated subgroup of B cells are memory B cells being defined as CD27 $7^{\text {var }}$ and CD38. DMF had a suppressing impact on their frequency: The proportion of memory B cells within all B cells was significantly reduced by DMF treatment (Figure 16). The mean 
frequency relatively decreased upon DMF treatment by $49.1 \%$, suggesting a strong, overproportionate memory B cell loss.

\subsubsection{The plasmablast frequency was decreased upon DMF treatment}

Plasmablasts are the progenitors of antibody-producing plasma cells and are defined as $\mathrm{CD} 20^{-}, \mathrm{CD} 27^{+}$and $\mathrm{CD} 38^{+}$. In general, they are found in very low frequencies within the $\mathrm{B}$ cell pool $(<8 \%)$. Figure 17 shows that in vivo DMF exposure caused a non-significant decrease in their mean frequency from $2.17 \%$ to $1.48 \%$ of all $\mathrm{B}$ cells.

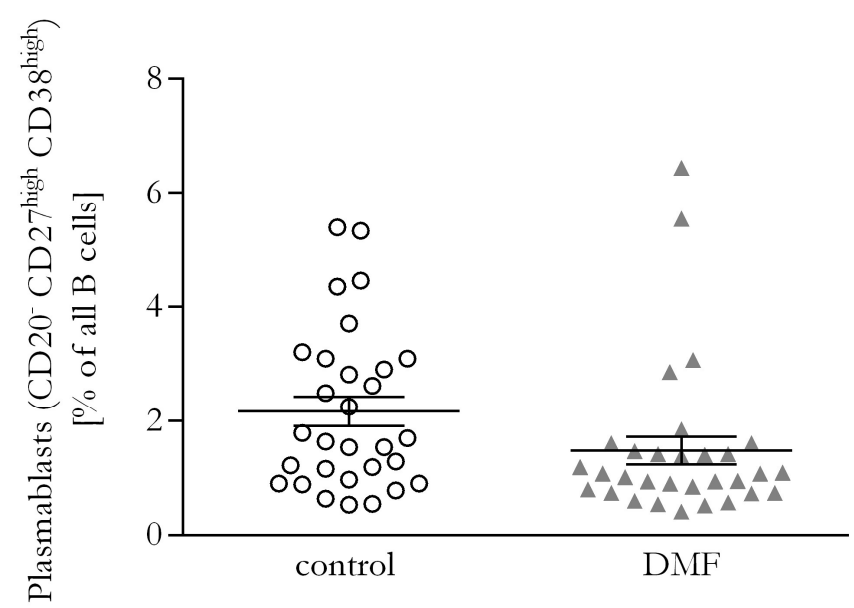

Figure 17: Dimethyl fumarate treatment reduced the frequency of plasmablasts. Plasmablast (CD20- CD27high CD38high) frequency of not stimulated pre-gated $\mathrm{BC}\left(\mathrm{CD} 19^{+}\right)$was measured using flow cytometry. Control multiple sclerosis patients (circles) were compared to dimethyl fumarate (DMF)-treated matches (triangles). Bars indicate mean \pm standard error of the mean. ( $n=31$ (DMF); $n=30$ (control); unpaired t-test; not significant)

Summarizing, especially the proportion of differentiated B cell subsets were reduced upon DMF, treatment while the frequency of immature transitional B cells was increased.

Table 13: Summary of $B$ cell subset changes. $(S D=$ standard deviation; $D M F=$ dimethyl fumarate; $\mathrm{BC}=\mathrm{B}$ cells; Ag-exp. = antigen-experienced; $\mathrm{ns}=$ not significant $)$

\begin{tabular}{|l|c|c|c|c|c|}
\hline & $\begin{array}{c}\text { Control } \\
\text { (mean } \pm \text { SD) }\end{array}$ & $\begin{array}{c}\text { DMF-treated } \\
\text { (mean } \pm \text { SD) }\end{array}$ & $\begin{array}{c}\text { Percental } \\
\text { change }\end{array}$ & $\begin{array}{c}\mathbf{p} \\
\text { value }\end{array}$ & \\
\hline Transitional BC (\% of BC) & $5.42 \pm 0.85$ & $9.59 \pm 1.10$ & $+76.9 \%$ & 0.0038 & $* *$ \\
Mature BC (\% of BC) & $84.04 \pm 0.90$ & $80.48 \pm 0.85$ & $-4.2 \%$ & 0.0059 & $* *$ \\
Ag-exp. BC (\% of BC) & $23.98 \pm 1.57$ & $12.21 \pm 1.02$ & $-49.1 \%$ & 0.0001 & $* * * *$ \\
Memory BC (\% of BC) & $17.82 \pm 1.28$ & $7.74 \pm 0.79$ & $-56.6 \%$ & 0.0001 & $* * * *$ \\
Plasmablasts (\% of BC) & $2.17 \pm 0.26$ & $1.48 \pm 0.24$ & $-31.8 \%$ & 0.0560 & $\mathrm{~ns}$ \\
\hline
\end{tabular}




\subsection{Activation markers}

To investigate whether DMF treatment changes the phenotype and possibly function of human B cells in vivo, the expression of activation markers was determined in DMF-treated and control-treated patients using flow cytometry. Therefore, total PBMC were stimulated with CpG and the frequency and/or normalized MFI of CD25, CD69, CD95 and CD150 expression were determined.

\subsubsection{DMF reduced the expression of CD25 on B cells}

PBMC were simulated with $2 \mu \mathrm{g} / \mathrm{ml} \mathrm{CpG}$ for 20 hours to increase the expression level of CD25 on B cells. In samples of DMF-treated patients, the expression of this IL-2 receptor chain was significantly lower with a relative decrease in the mean MFI values by $49.3 \%$. This decrease was also seen when looking at the $\mathrm{CD} 25^{+} \mathrm{B}$ cell frequencies (Figure 18).
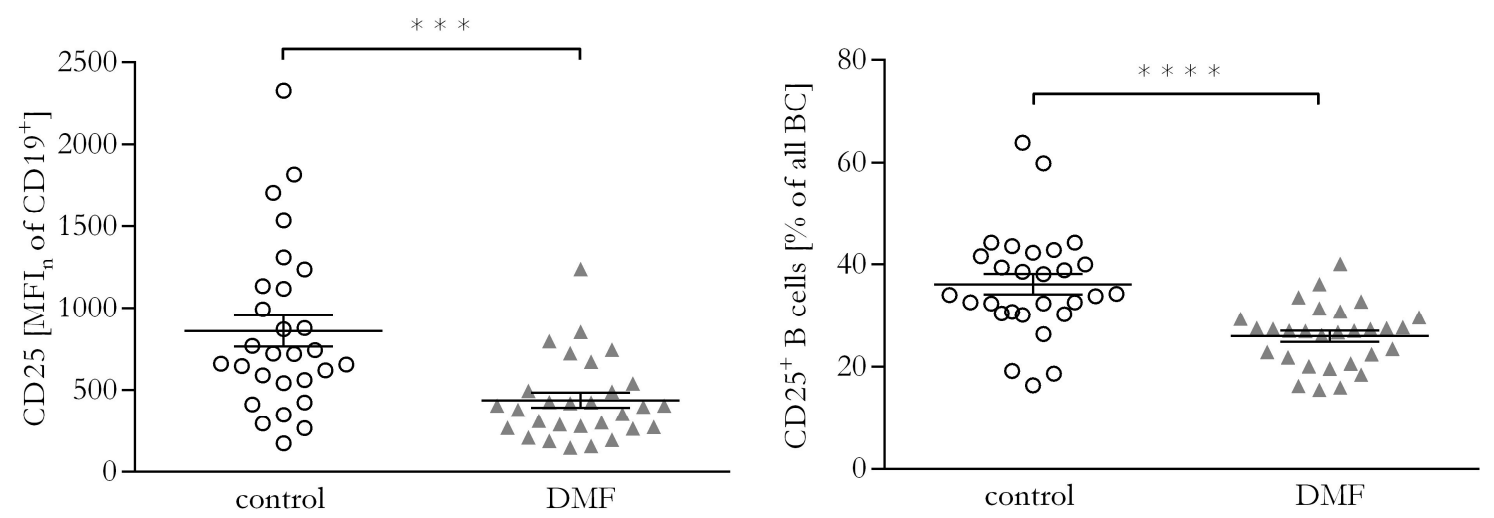

Figure 18: CD25 expression on B cells was reduced by dimethyl fumarate treatment. Peripheral blood mononuclear cells were stimulated with $2 \mu \mathrm{g} / \mathrm{ml}$ cytosine-phosphate-guanine for 20 hours. The expression of the activation marker CD25 was quantified on B cells $(\mathrm{BC})\left(\mathrm{CD} 19^{+}\right)$by flow cytometry given by the normalized mean fluorescent intensity $\left(\mathrm{MFI}_{\mathrm{n}}\right)($ left graph). In addition, the frequency of $\mathrm{CD} 25^{+} \mathrm{BC}$ was determined (left graph). Control multiple sclerosis patients (circles) were compared to dimethyl fumarate (DMF)-treated matches (triangles). Bars indicate mean \pm standard error of the mean. $(n=31(\mathrm{DMF}) ; n=30$ (control); unpaired t-test; $* * *=\mathrm{p}<0.001 ; * * * *=\mathrm{p}<0.0001)$

\subsubsection{DMF treatment diminished the expression of the early activation marker CD69 on B cells}

DMF-treated patients had a significant lower percentage of $\mathrm{CD} 69^{+} \mathrm{B}$ cells in their blood compared to control-treated patients (Figure 19). The mean $\mathrm{CD} 69^{+} \mathrm{B}$ cell frequency decreased from $33.85 \pm 2.32 \%$ to $27.13 \pm 1.82 \%$ in the DMF-treated group. This represents a relative reduction by $23.4 \%$. The MFI values showed a similar trend. 

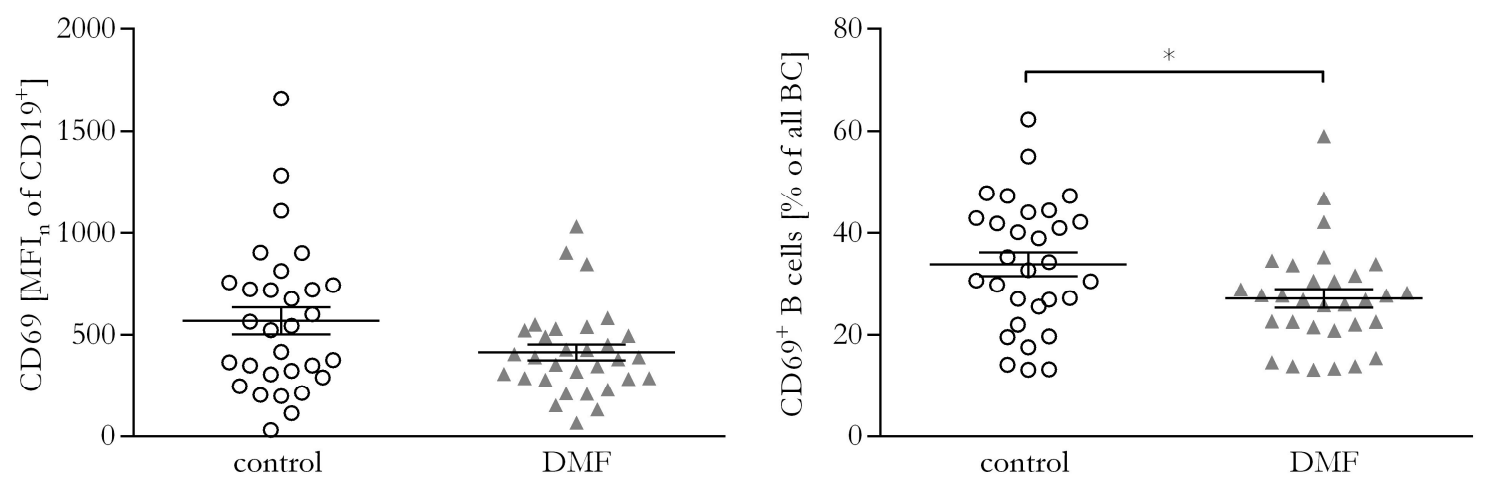

Figure 19: CD69 was reduced by dimethyl fumarate on cytosine-phosphate-guanine-stimulated B cells. Peripheral blood mononuclear cells were stimulated with $2 \mu \mathrm{g} / \mathrm{ml}$ cytosine-phosphate-guanine for 20 hours. The expression of the activation marker $\mathrm{CD} 69$ was quantified on $\mathrm{B}$ cells $(\mathrm{BC})\left(\mathrm{CD} 19^{+}\right)$by flow cytometry given by the normalized mean fluorescent intensity $\left(\mathrm{MFI}_{\mathrm{n}}\right)$ (left graph). In addition, the frequency of $\mathrm{CD} 69^{+} \mathrm{BC}$ was determined (left graph). Control multiple sclerosis patients (circles) were compared to dimethyl fumarate (DMF)-treated matches (triangles). Bars indicate mean \pm standard error of the mean. $(n=31(\mathrm{DMF}) ; n=30$ (control); unpaired t-test; $*=\mathrm{p}<0.05)$

\subsubsection{Lower expression of the FAS receptor CD95 on B cells of DMF-treated patients}

Furthermore, MFI values of CD95 (FAS receptor) expression on both unstimulated and stimulated B cells were quantified. In both conditions, this important protein for controlled cell death was lower on B cells of DMF-treated patients (Figure 20). CpG stimulation caused an upregulation in both groups but the significant difference persisted.

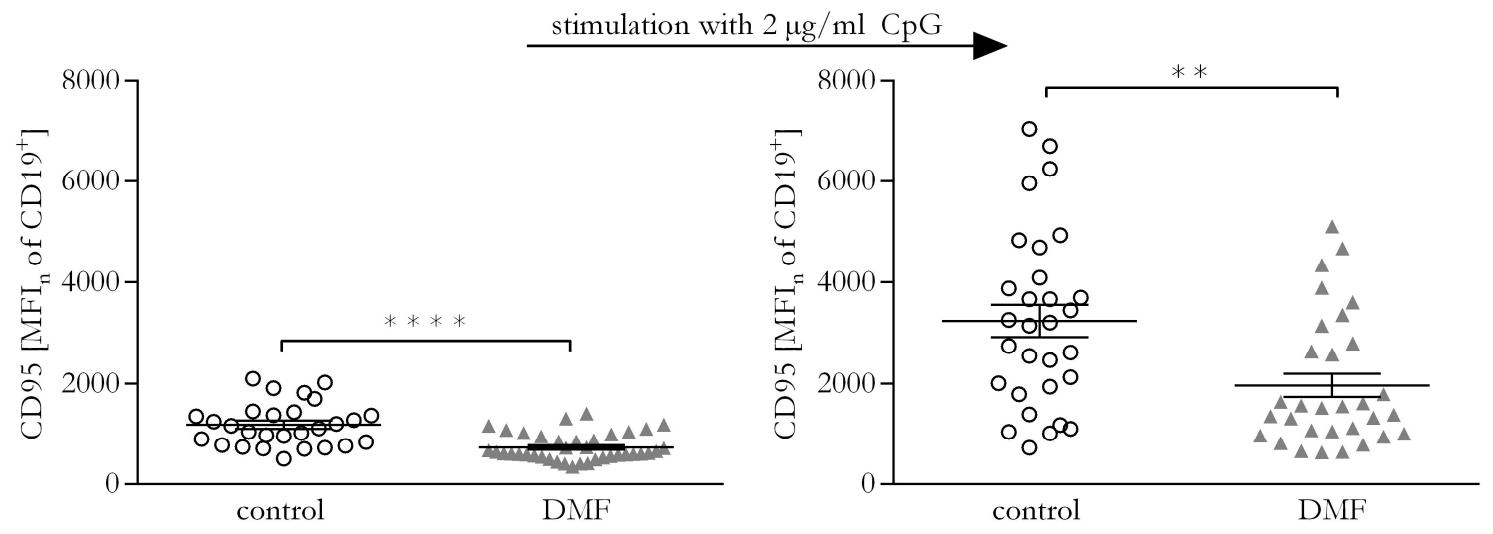

Figure 20: Dimethyl fumarate reduced the expression of CD95 on B cells. Peripheral blood mononuclear cells were stimulated with $2 \mu \mathrm{g} / \mathrm{ml}$ cytosine-phosphate-guanine (CpG) for 20 hours. The expression of the activation marker CD95 (first apoptosis signal receptor) was quantified on both not stimulated (left graph) and stimulated (right graph) B cells (BC) $\left(\mathrm{CD} 19^{+}\right)$by flow cytometry given by the normalized mean fluorescent intensity $\left(\mathrm{MFI}_{\mathrm{n}}\right)$. Control multiple sclerosis patients (circles) were compared to dimethyl fumarate (DMF)-treated matches (triangles). Bars indicate mean \pm standard error of the mean. $(n=31$ (DMF); $n=30$ (control); unpaired t-test; ** $=\mathrm{p}<0.01 ; * * * *=\mathrm{p}<0.0001)$

\subsubsection{DMF had an inhibitory impact on CD150 expression on B cells}

Next, the expression of CD150, a molecule which acts as a co-activator on B cells, was examined. No significant difference was observed in unstimulated patient samples (Figure 21). LPS stimulation however slightly upregulated this surface protein, especially in the samples of control-treated MS patients, uncovering a significant difference between both 
patient groups. In the control group, $15.51 \pm 1.47 \%$ B cells were CD150 ${ }^{+}$upon LPS stimulation, while in DMF-treated individuals, only $11.70 \pm 0.93 \%$ of the B cells expressed CD150.
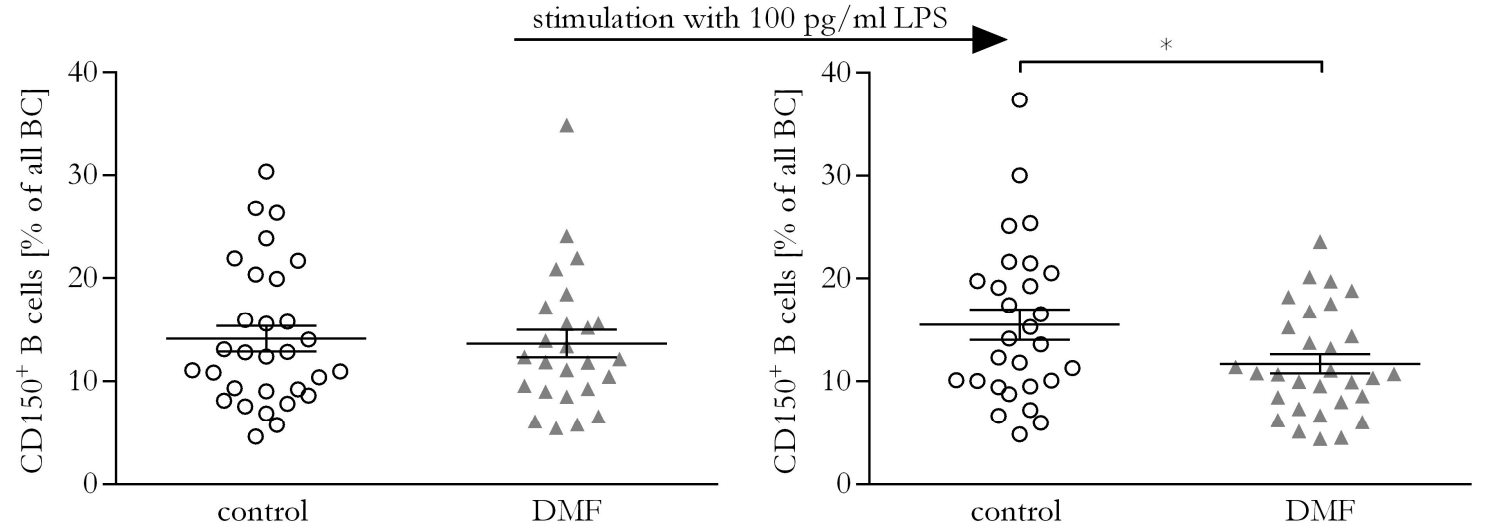

Figure 21: $\mathrm{CD} 150^{+} \mathrm{B}$ cells were reduced upon dimethyl fumarate treatment. Peripheral blood mononuclear cells were stimulated with $100 \mathrm{pg} / \mathrm{ml}$ lipopolysaccharide (LPS) for 20 hours. The frequency of CD150+ B cells (BC) was quantified on both not stimulated (left graph) and stimulated (right graph) B cells (BC) (CD19+). Control multiple sclerosis patients (circles) were compared to dimethyl fumarate (DMF)-treated matches (triangles). Bars indicate mean \pm standard error of the mean. $(n=31(\mathrm{DMF}) ; n=30$ (control); unpaired t-test; $*=\mathrm{p}<0.05)$

Table 14: Summary of dimethyl fumarate-induced changes of B cell activation markers. (SD = standard deviation; $\mathrm{DMF}=$ dimethyl fumarate; $\mathrm{CD}=$ cluster of differentiation; $\mathrm{MFI}=$ mean fluorescent intensity; $\mathrm{CpG}=$ cytosine-phosphate-guanine; $\mathrm{LPS}=$ lipopolysaccharide)

\begin{tabular}{|l|c|c|c|c|c|c|}
\hline & $\begin{array}{c}\text { stimu- } \\
\text { lation }\end{array}$ & $\begin{array}{c}\text { control (mean } \\
\mathbf{\pm} \text { SD) }\end{array}$ & $\begin{array}{c}\text { DMF-treated } \\
\text { (mean } \pm \text { SD) }\end{array}$ & $\begin{array}{c}\text { percental } \\
\text { change }\end{array}$ & $\begin{array}{c}\mathbf{p} \\
\text { value }\end{array}$ & \\
\hline CD25 [MFI] & CpG & $860.0 \pm 96.68$ & $436.3 \pm 45.92$ & $-49.3 \%$ & 0.0002 & $* * *$ \\
CD69+ [\%] & CpG & $33.85 \pm 2.32$ & $27.13 \pm 1.82$ & $-19.9 \%$ & 0.0258 & $*$ \\
CD95 [MFI] & CpG & $3238 \pm 319.4$ & $1963 \pm 23.8$ & $-39.4 \%$ & 0.0019 & $* *$ \\
CD150+ [\%] & LPS & $15.51 \pm 1.47$ & $11.70 \pm 0.93$ & $-24.6 \%$ & 0.0306 & $*$ \\
\hline
\end{tabular}

\subsubsection{DMF treatment did not alter activation markers on myeloid-derived phagocytes}

Furthermore, this examination quantified the expression of the activation markers CD95 and CD150 on $\mathrm{CD}^{+} 4^{+}$myeloid APC. As shown in Figure 22, their expression was not significantly altered upon DMF treatment. 

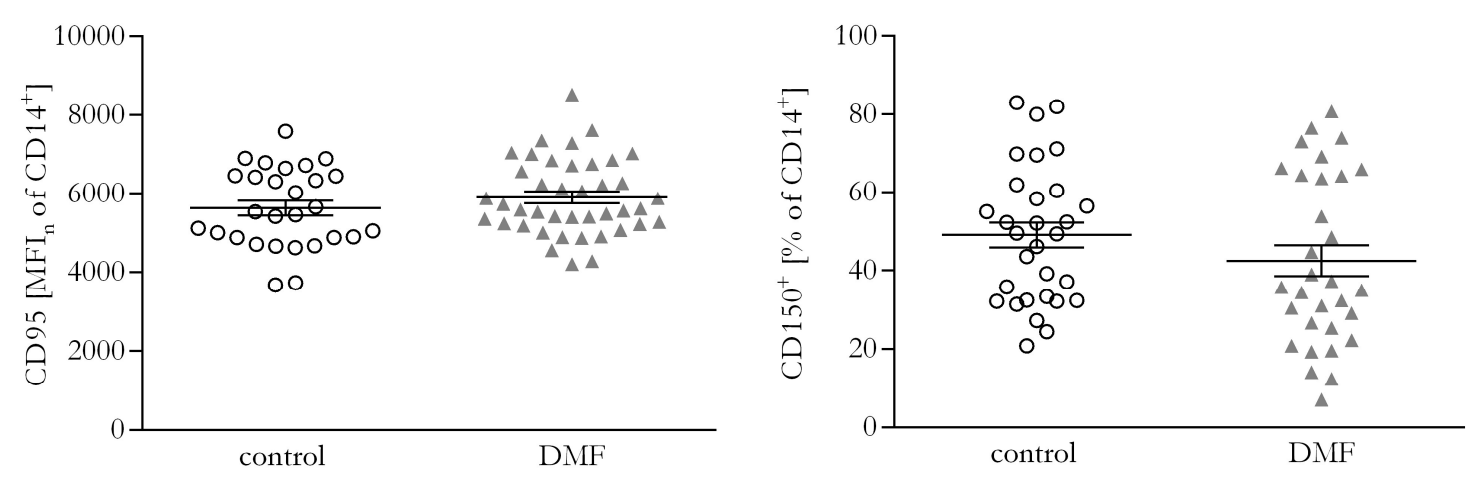

Figure 22: Dimethyl fumarate did not alter the expression of activation markers on myeloid phagocytes. On the left, peripheral blood mononuclear cells were stimulated with $2 \mu \mathrm{g} / \mathrm{ml}$ cytosine-phosphate-guanine $(\mathrm{CpG})$ for 20 hours. The expression of the activation marker CD95 (first apoptosis signal receptor) was quantified on myeloid phagocytes (CD14+) by flow cytometry given by the normalized mean fluorescent intensity $\left(\mathrm{MFI}_{\mathrm{n}}\right)$. The left graph presents the frequency of CD150+ myeloid phagocytes after a stimulation of $100 \mathrm{pg} / \mathrm{ml}$ lipopolysaccharide (LPS) for 20 hours. Control multiple sclerosis patients (circles) were compared to dimethyl fumarate (DMF)-treated matches (triangles). Bars indicate mean \pm standard error of the mean. ( $n=31$ (DMF); $n=30$ (control); unpaired t-test; not significant)

\subsection{Antigen-presenting function}

To evaluate the ability of B cells and myeloid-derived phagocytes to present antigens, it was tested whether DMF treatment influences the expression of molecules relevant for antigen presentation such as MHC-II, CD40, CD80 and CD86.

\subsubsection{MHC-II expression on B cells was enhanced by DMF treatment}

The MFI values of MHC-II were quantified using the standard protocol. The cross-sectional analysis revealed no difference between both patient groups (Figure 23), neither in the unstimulated nor in the stimulated stetting, which indicates that DMF treatment did not alter its expression.

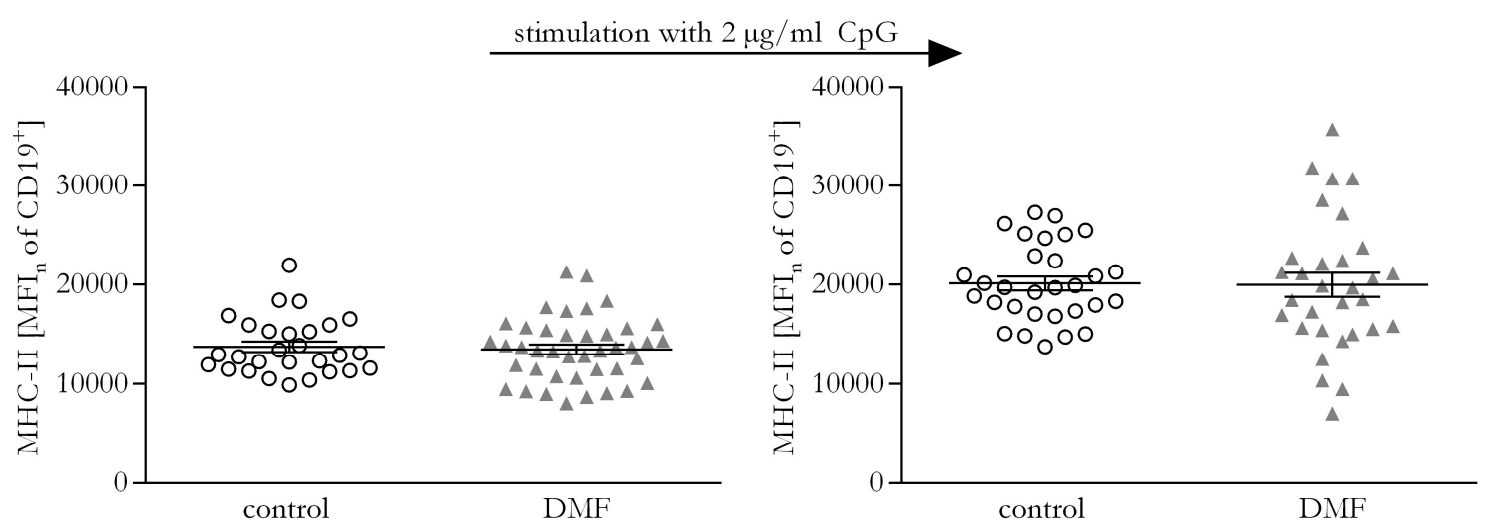

Figure 23: Major histocompatibility complex - class II expression on B cells was not altered by dimethyl fumarate. Peripheral blood mononuclear cells were stimulated with $2 \mu \mathrm{g} / \mathrm{ml}$ cytosine-phosphate-guanine (CpG) for 20 hours. The expression of major histocompatibility complex class II (MHC-II) was quantified on both not stimulated (left graph) and stimulated (right graph) B cells $(\mathrm{BC})\left(\mathrm{CD} 19^{+}\right)$by flow cytometry given by the normalized mean fluorescent intensity $\left(\mathrm{MFI}_{\mathrm{n}}\right)$. Control multiple sclerosis patients (circles) were compared to dimethyl fumarate (DMF)-treated matches (triangles). Bars indicate mean \pm standard error of the mean. ( $n=31$ (DMF); $n=30$ (control); unpaired t-test; not significant) 
When analyzing this parameter on B cells longitudinally, MHC-II expression was higher in patients taking DMF when compared to their own blood control prior to treatment initiation in the CpG stimulated setting (Figure 24).
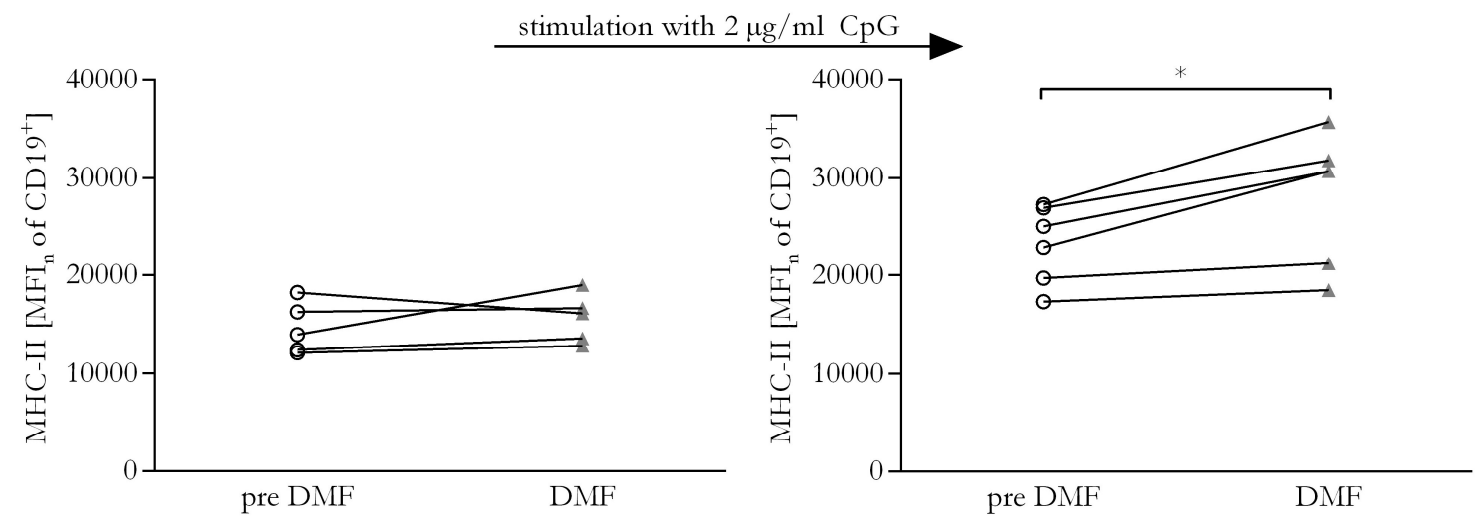

Figure 24: In the longitudinal samples, dimethyl fumarate upregulated major histocompatibility complex - class II on B cells. Peripheral blood mononuclear cells were stimulated with $2 \mu \mathrm{g} / \mathrm{ml}$ cytosine-phosphate-guanine (CpG) for 20 hours. The expression of major histocompatibility complex - class II (MHC-II) was quantified on both not stimulated (left graph) and stimulated (right graph) B cells $(\mathrm{BC})\left(\mathrm{CD} 19^{+}\right)$by flow cytometry given by the normalized mean fluorescent intensity $\left(\mathrm{MFI}_{\mathrm{n}}\right)$. Blood samples of the same patients were taken before the initiation of dimethyl fumarate (DMF) therapy (circles) and after at least 3 months of DMF treatment. Lines connect the values of individual patients. $(n=31$ (DMF); $n=30$ (control); Wilcoxon matched-pairs signed rank test; $*=\mathrm{p}<0.05$ )

Next, it was examined whether duration of DMF treatment influenced MHC-II expression on B cells. In fact, there was a trend towards a higher expression of MHC-II the longer DMF was taken (Figure 25), but this trend was not significant $(\mathrm{p}=0.138)$.

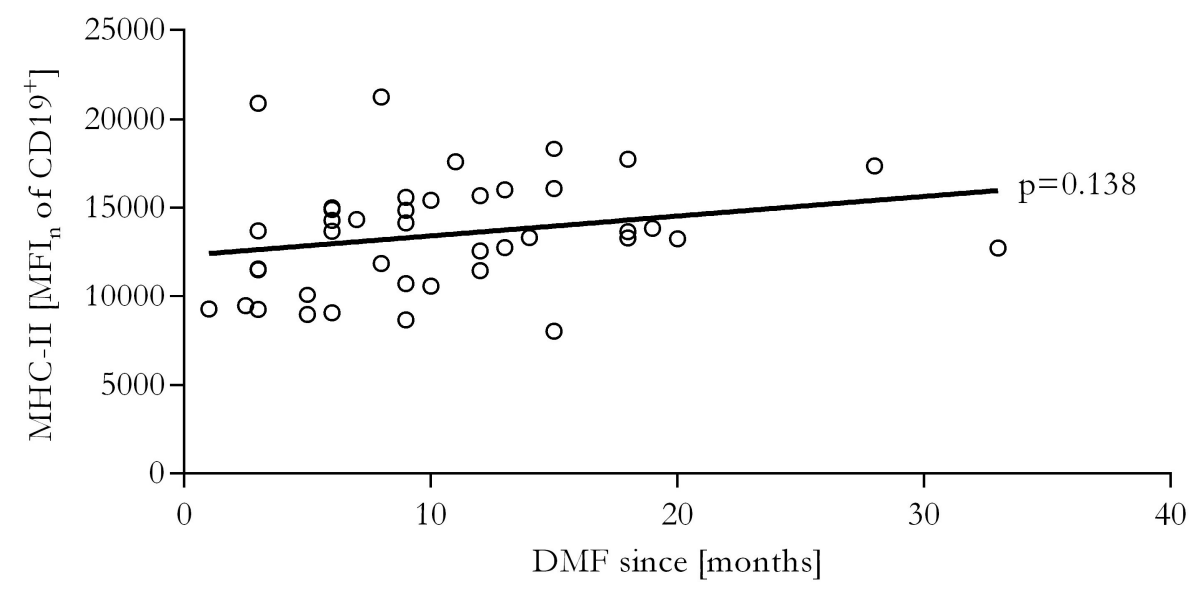

Figure 25: Major histocompatibility complex - class II on B cells tended to increase the longer dimethyl fumarate was taken. The expression of major histocompatibility complex - class II (MHC-II) was quantified on not stimulated B cells (BC) $\left(\mathrm{CD} 19^{+}\right)$. The individual patients' values (dimethyl fumarate (DMF)-treated patients are represented by circles) were correlated with the time in months they had taken DMF using linear regression. ( $n=31$ (DMF); Pearson correlation coefficient $(\mathrm{r})=0.2356 ; \mathrm{p}=0.138)$

\subsubsection{DMF treatment prevented the stimulation-triggered upregulation of CD40}

The expression of the costimulatory protein CD40 on B cells was measured both on unstimulated and $\mathrm{CPG}$ stimulated PBMC. As presented in Figure 26, its expression was 
comparable in both patient cohorts in the unstimulated setting but was differently upregulated upon stimulation. In DMF-treated subjects, B cells did not increase CD40 expression to the same extent as cells of control-treated patients. This resulted in a significant decline of CD40 MFI values by $19.8 \%$ in samples of treated patients compared to control subjects.
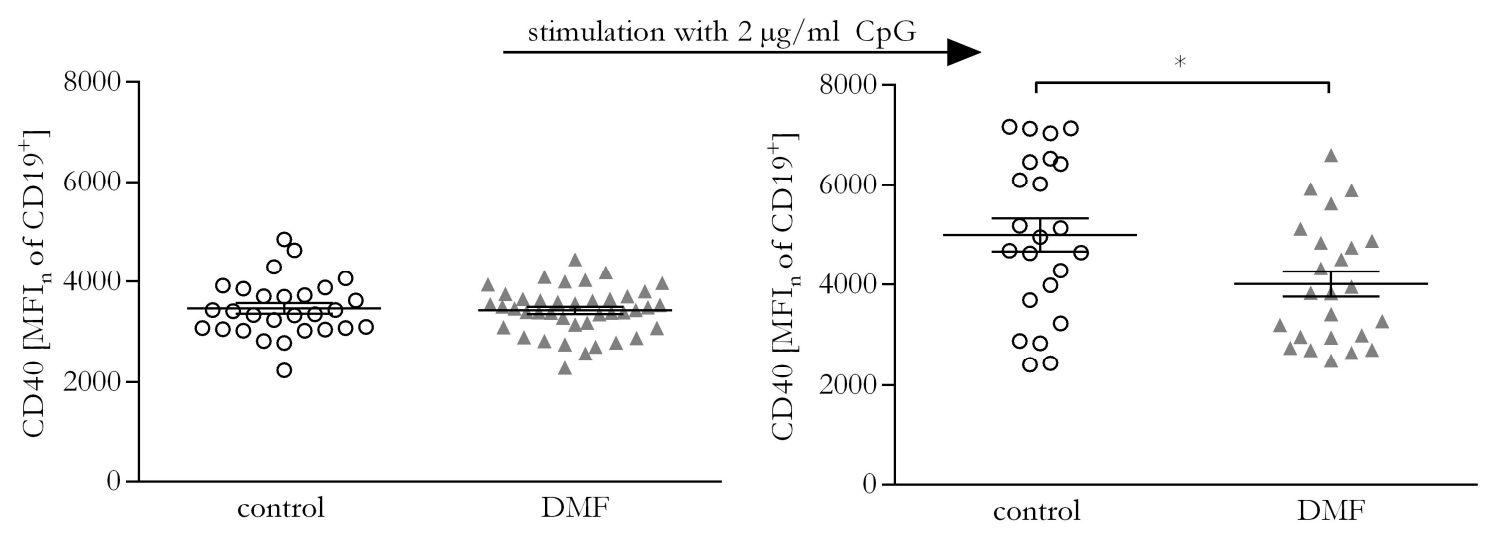

Figure 26: Upon stimulation, CD40 expression on B cells was reduced. Peripheral blood mononuclear cells were stimulated with $2 \mu \mathrm{g} / \mathrm{ml}$ cytosine-phosphate-guanine (CpG) for 20 hours. The expression of the activation marker CD40 was quantified on both not stimulated (left graph) and stimulated (right graph) B cells (BC) (CD19+) by flow cytometry given by the normalized mean fluorescent intensity $\left(\mathrm{MFI}_{\mathrm{n}}\right)$. Control multiple sclerosis patients (circles) were compared to dimethyl fumarate (DMF)-treated matches (triangles). Bars indicate mean \pm standard error of the mean. $(n=31$ (DMF); $n=30$ (control); unpaired t-test; $*=p<0.05$ )

\subsubsection{Independent of the stimulation, CD80 was reduced on B cells by DMF}

Another important costimulatory signal during B cell - T cell interaction is provided through CD80, also called lymphocyte-activating antigen 1 . In this study, its expression was measured in an unstimulated as well as a stimulated setting. Figure 27 shows that a significant difference between PBMC of DMF- and control-treated patients was already evident without stimulation. CpG stimulation induced an upregulation of CD80 in samples of both patient groups, but the significantly lower CD80 expression in DMF-treated patients remained present. In the $\mathrm{CpG}$ stimulated condition, control patients had a mean MFI of 441.5 \pm 36.5 , whereas DMF treatment lead to a mean of $279.2 \pm 31.5$. This corresponds to a reduction by $36.8 \%$. 


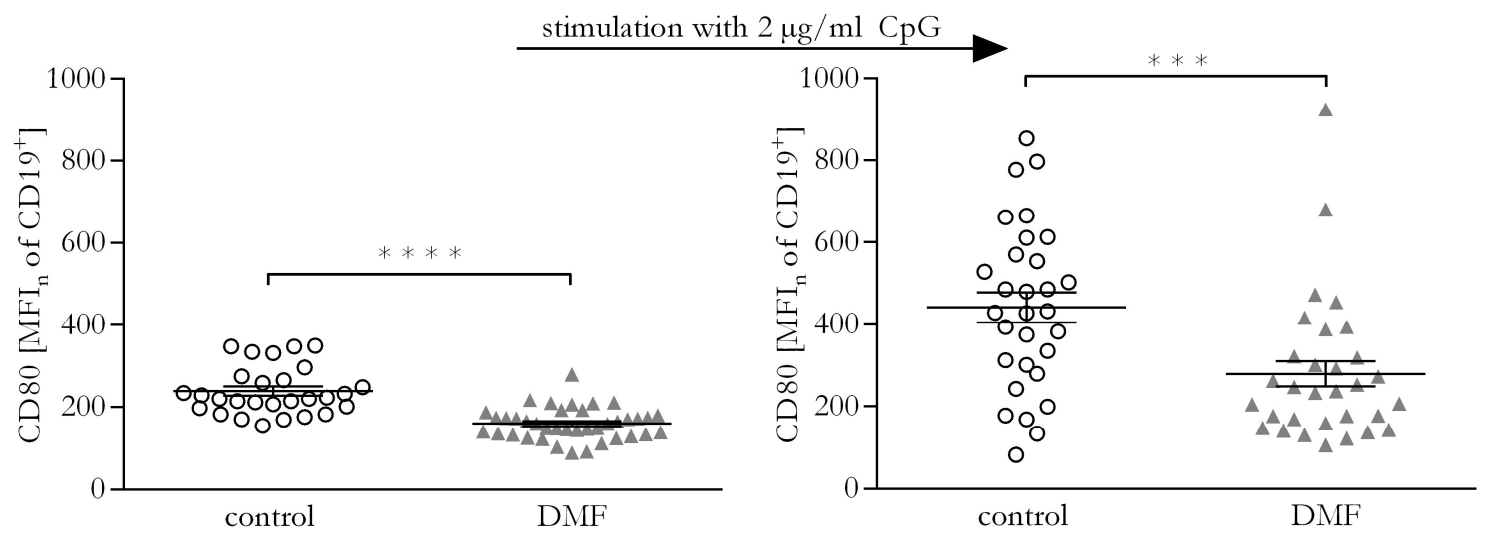

Figure 27: Dimethyl fumarate reduced the expression of CD80 on B cells. Peripheral blood mononuclear cells were stimulated with $2 \mu \mathrm{g} / \mathrm{ml}$ cytosine-phosphate-guanine ( $\mathrm{CpG}$ ) for 20 hours. The expression of the activation marker CD80 was quantified on both not stimulated (left graph) and stimulated (right graph) B cells (BC) (CD19+) by flow cytometry given by the normalized mean fluorescent intensity $\left(\mathrm{MFI}_{\mathrm{n}}\right)$. Control multiple sclerosis patients (circles) were compared to dimethyl fumarate (DMF)-treated matches (triangles). Bars indicate mean \pm standard error of the mean. $(n=31$ (DMF); $n=30$ (control); unpaired t-test; $* * *=\mathrm{p}<0.001 ; * *=\mathrm{p}<0.0001)$

\subsubsection{DMF restricts the upregulation of the costimulatory protein CD86 on B cells}

The expression of CD86 was very low on unstimulated B cells with no significant difference between both patient groups. Stimulation with $2 \mu \mathrm{g} / \mathrm{ml} \mathrm{CpG}$ however considerably increased the surface expression of CD86 and showed that DMF-treated patients in comparison to the control cohort had significant lower MFI values with a relative reduction by $48.3 \%$ (Figure 28).

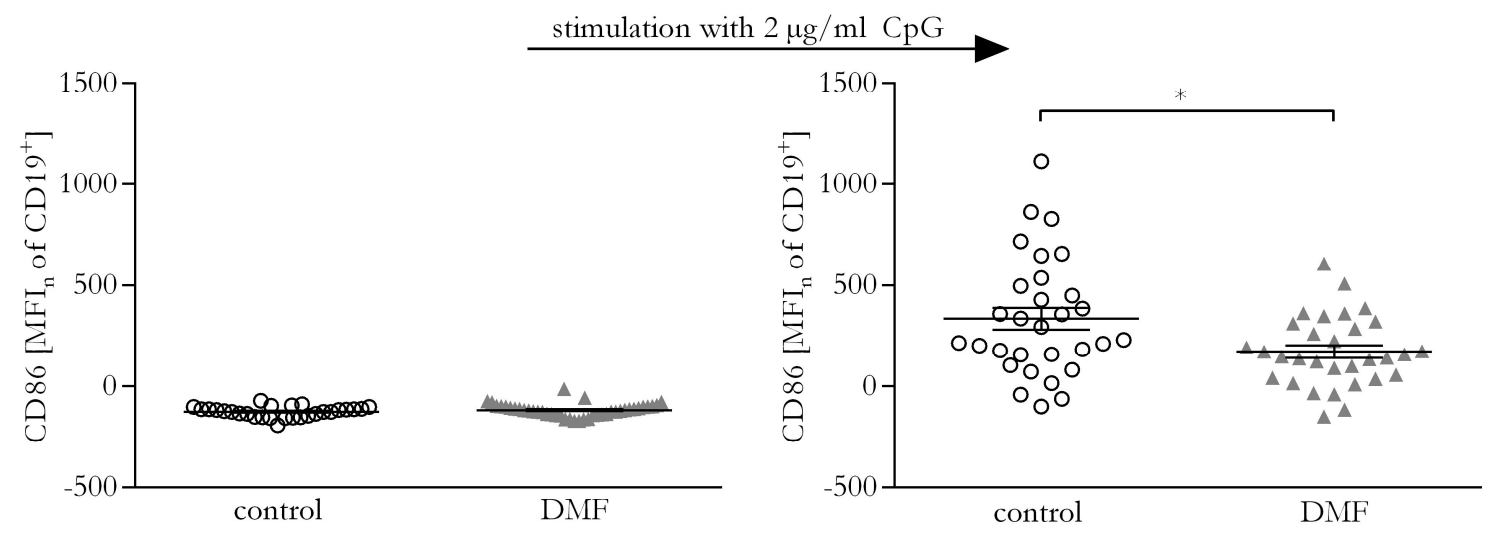

Figure 28: CD86 upregulation upon stimulation was inhibited by dimethyl fumarate. Peripheral blood mononuclear cells were stimulated with $2 \mu \mathrm{g} / \mathrm{ml}$ cytosine-phosphate-guanine (CpG) for 20 hours. The expression of the activation marker CD80 was quantified on both not stimulated (left graph) and stimulated (right graph) B cells (BC) (CD19+) by flow cytometry given by the normalized mean fluorescent intensity $\left(\mathrm{MFI}_{\mathrm{n}}\right)$. Control multiple sclerosis patients (circles) were compared to dimethyl fumarate (DMF)-treated matches (triangles). Bars indicate mean \pm standard error of the mean. $(n=31(\mathrm{DMF}) ; n=30$ (control); unpaired t-test; $*=\mathrm{p}<0.05)$ 
Table 15: Dimethyl fumarate-induced changes of molecules involved in antigen presentation on $B$ cells. $(\mathrm{SD}=$ standard deviation; $\mathrm{DMF}=$ dimethyl fumarate; $\mathrm{CD}=$ cluster of differentiation; $\mathrm{MFI}=$ mean fluorescent intensity; $\mathrm{CpG}=$ cytosine-phosphate-guanine; $\mathrm{MHC}$-II = major histocompatibility complex - class II; ns $=$ not significant $)$

\begin{tabular}{|l|c|c|c|c|c|c|}
\hline & $\begin{array}{c}\text { stimu- } \\
\text { lation }\end{array}$ & $\begin{array}{c}\text { control (mean } \\
\mathbf{\pm} \text { SD) }\end{array}$ & $\begin{array}{c}\text { DMF-treated } \\
\text { (mean } \pm \text { SD) }\end{array}$ & $\begin{array}{c}\text { percental } \\
\text { change }\end{array}$ & $\begin{array}{c}\mathbf{p} \\
\text { value }\end{array}$ & \\
\hline CD40 [MFI] & $\mathrm{CpG}$ & $4999 \pm 332.1$ & $4009 \pm 248.4$ & $-19.8 \%$ & 0.0205 & $*$ \\
CD80 [MFI] & $\mathrm{CpG}$ & $441.5 \pm 36.52$ & $279.2 \pm 31.45$ & $-36.8 \%$ & 0.0013 & $* * *$ \\
CD86 [MFI] & $\mathrm{CpG}$ & $335.9 \pm 53.79$ & $173.5 \pm 31.28$ & $-48.3 \%$ & 0.0109 & $*$ \\
MHC-II [MFI] & $\mathrm{CpG}$ & $20114 \pm 715.8$ & $19975 \pm 1210$ & $-0.7 \%$ & 0.9224 & $\mathrm{~ns}$ \\
\hline
\end{tabular}

In summary, DMF treatment influenced the expression of molecules required for antigen presentation differentially. While the costimulatory molecules CD40, CD80 and CD86 were downregulated by DMF treatment, the important protein for antigen presentation, MHC-II, remained unchanged or was slightly upregulated upon DMF exposure.

\subsubsection{DMF increased MHC-II expression on myeloid APC over time}

Next to B cells, myeloid-derived phagocytes are important APC in the context of MS. Thus, the expression of MHC-II was analyzed on CD14 ${ }^{+}$monocytes/macrophages (Figure 29), but no significant alteration was found.
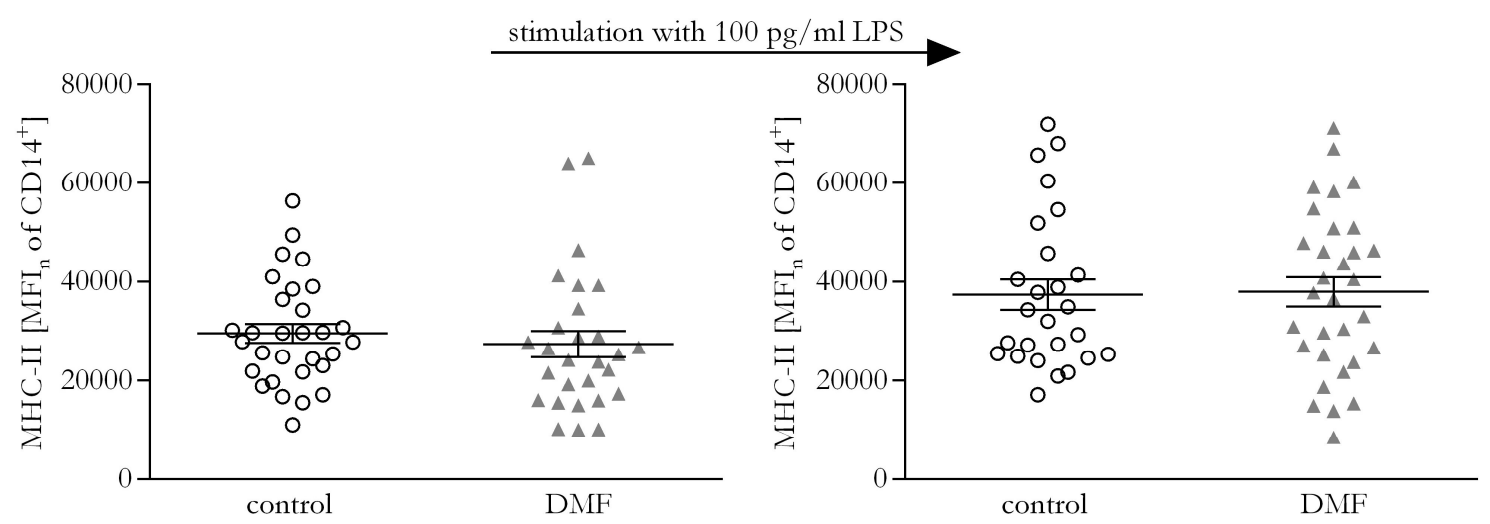

Figure 29: Dimethyl fumarate did not change the major histocompatibility complex - class II expression on myeloidderived phagocytes. Peripheral blood mononuclear cells were stimulated with $100 \mathrm{pg} / \mathrm{ml}$ lipopolysaccharide (LPS) for 20 hours. The expression of major histocompatibility complex - class II (MHC-II) was quantified on both not stimulated (left graph) and stimulated (right graph) myeloid-derived phagocytes $\left(\mathrm{CD} 14^{+}\right)$by flow cytometry given by the normalized mean fluorescent intensity $\left(\mathrm{MFI}_{\mathrm{n}}\right)$. Control multiple sclerosis patients (circles) were compared to dimethyl fumarate (DMF)-treated matches (triangles). Bars indicate mean \pm standard error of the mean. $(n=31$ (DMF); $n=30$ (control); unpaired t-test; not significant) 
However, when correlating the duration of DMF intake to the expression of MHC-II on myeloid-derived phagocytes, there was a significant increase of MHC-II the longer DMF had been taken (Figure 30).

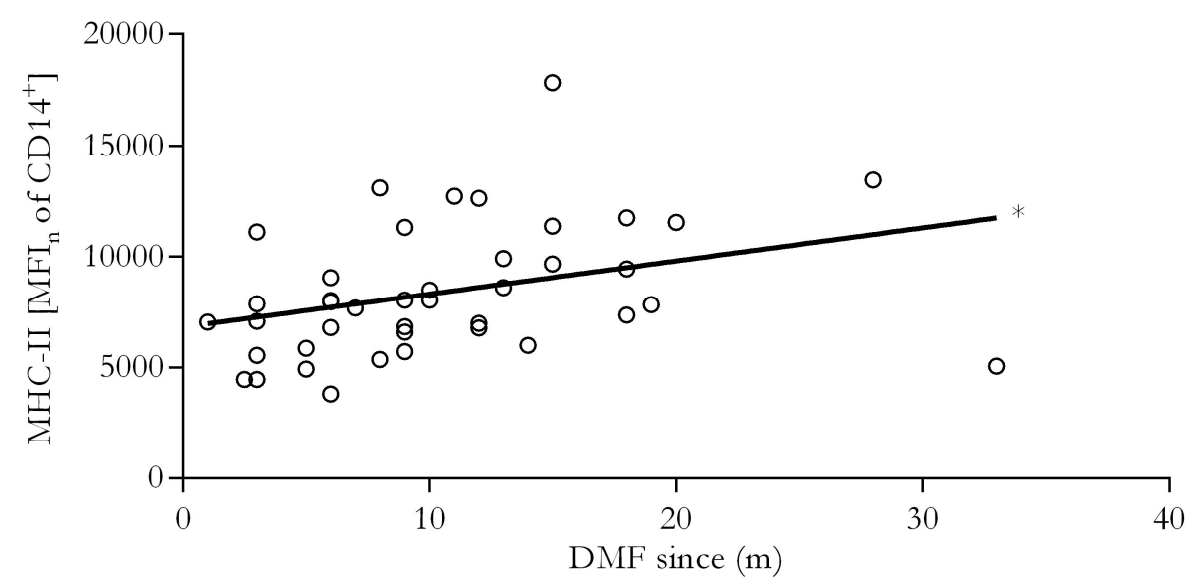

Figure 30: The expression of major histocompatibility complex - class II positively correlated with the duration of dimethyl fumarate intake. The expression of major histocompatibility complex - class II (MHC-II) was quantified on not stimulated myeloid phagocytes $\left(\mathrm{CD} 14^{+}\right)$. The individual patients' values (dimethyl fumarate (DMF)-treated patients are represented by circles) were correlated with the time in months they had taken DMF using linear regression. $(n=31$ (DMF); Pearson correlation coefficient $(\mathrm{r})=0.3421 ; *=\mathrm{p}<0.05)$

\subsubsection{The expression of CD80 on myeloid phagocytes was reduced by DMF}

Further evaluation of the antigen-presenting ability of myeloid-derived phagocytes $\left(\mathrm{CD} 14^{+}\right)$ was performed by comparing the expression of the costimulatory molecules CD40, CD80 and CD86. In an unstimulated condition, only CD80 expression was significantly reduced upon DMF treatment, while the other markers remained unaltered as shown in Figure 31.
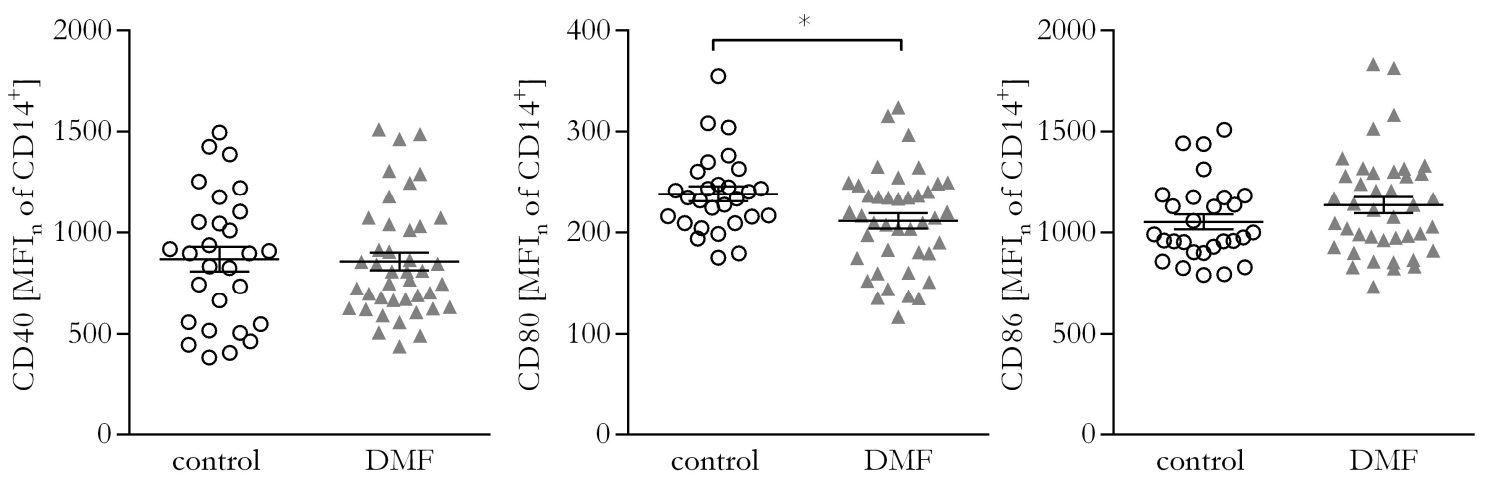

Figure 31: Dimethyl fumarate reduced the expression of CD80 on myeloid-derived phagocytes. The expression of the activation markers CD40, CD80 and CD86 was quantified on not stimulated myeloid phagocytes (CD14 ${ }^{+}$) by flow cytometry given by the normalized mean fluorescent intensity $\left(\mathrm{MFI}_{\mathrm{n}}\right)$. Control multiple sclerosis patients (circles) were compared to dimethyl fumarate (DMF)-treated matches (triangles). Bars indicate mean \pm standard error of the mean. $(n=31(\mathrm{DMF}) ; n=30$ (control); unpaired t-test; $*=\mathrm{p}<0.05)$ 


\subsection{Cytokine production}

The effect of DMF on the cytokine production of B cells and myeloid cells was analyzed by performing intracellular staining for cytokines as described in 2.3.6. Using flow cytometry, the cellular cytokine content was quantified by determining the MFI of the respective fluorescence-labeled antibody. In a second approach, the cytokine concentration in the supernatant was measured using ELISA to substantiate the flow cytometry-based results. This study focused on the two pro-inflammatory cytokines IL-6 and TNF, and one antiinflammatory, namely IL-10.

\subsubsection{DMF reduced the production of pro-inflammatory IL-6 and TNF in B cells}

To make the B cells produce detectable levels of cytokines, cells were pre-stimulated with $1 \mu \mathrm{g} / \mathrm{ml} \mathrm{CpG} \mathrm{overnight} \mathrm{and} \mathrm{after} \mathrm{inhibition} \mathrm{of} \mathrm{the} \mathrm{Golgi} \mathrm{apparatus,} \mathrm{cytokine} \mathrm{production} \mathrm{was}$ boosted by adding $500 \mathrm{ng} / \mathrm{ml}$ ionomycin and $20 \mathrm{ng} / \mathrm{ml}$ PMA. Upon DMF treatment, a reduction of pro-inflammatory IL-6 synthesis by B cells was observed (Figure 32). This statistically significant decline was seen in both cross-sectional as well as longitudinal samples. There was a reduction of MFI values from a mean of 1032.7 to 788.1 in the cross-sectional samples, equivalent to a solid reduction by $23.7 \%$. With a mean reduction by $60.4 \%$, this effect was even stronger in the longitudinal samples.
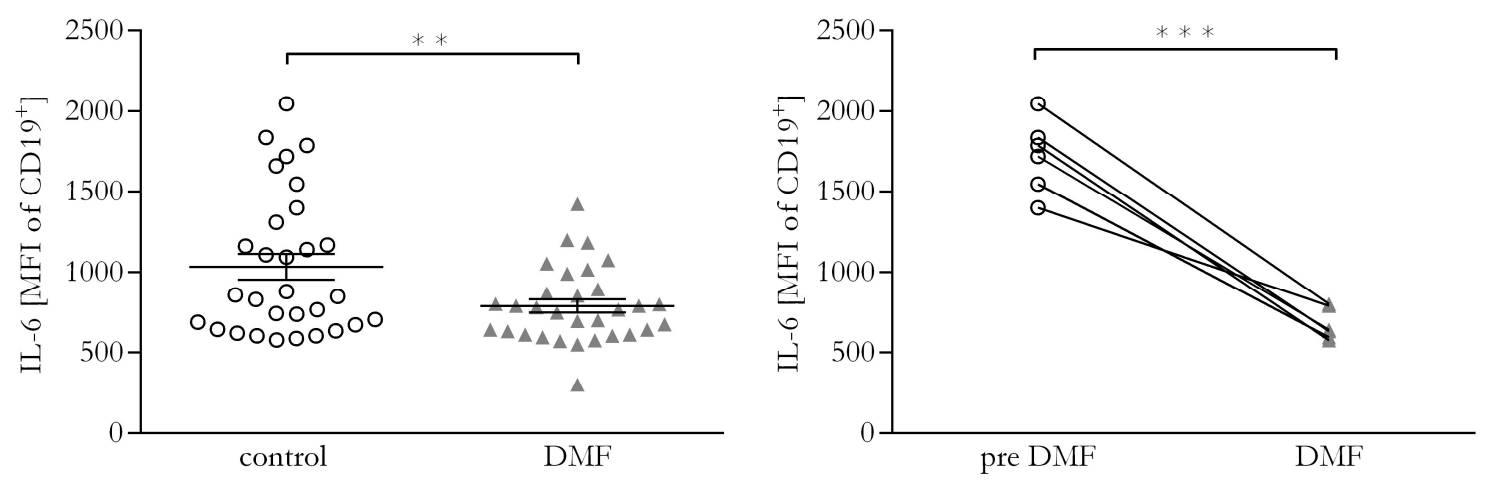

Figure 32: Interleukin-6 production of B cells was reduced upon dimethyl fumarate treatment. Peripheral blood mononuclear cells were stimulated with $1 \mu \mathrm{g} / \mathrm{ml}$ cytosine-phosphate-guanine for 20 hours and $500 \mathrm{ng} / \mathrm{ml}$ ionomycin / $20 \mathrm{ng} / \mathrm{ml}$ phorbol 12-myristate 12 -acetate for 4 hours. The production of interleukin-6 (IL-6) by B cells (CD19+) was quantified by flow cytometric intracellular staining given by the normalized mean fluorescent intensity $\left(\mathrm{MFI}_{\mathrm{n}}\right)$. In a cross-sectional setting, control multiple sclerosis patients (circles) were compared to dimethyl fumarate (DMF)-treated matches (triangles) (left graph). In addition, six patients' blood samples were taken before the initiation of DMF therapy (circles) and after at least 6 months of DMF treatment (triangles). Lines connect the values of individual patients. Bars indicate mean \pm standard error of the mean. $(n=31$ (DMF); $n=30$ (control); $n=6$ (longitudinal); unpaired t-test (cross-sectional); Wilcoxon matched-pairs signed rank test (longitudinal); ** $=\mathrm{p}<0.01$; *** $=\mathrm{p}<0.001$ )

Next, the production of TNF by B cells was evaluated, as it is another important proinflammatory cytokine involved in pathogenic immune responses. The cross-sectional data analysis revealed no significant difference between control and DMF-treated patients regarding TNF synthesis. In the longitudinal samples however, there was a highly significant decline in the production of TNF (Figure 33). 

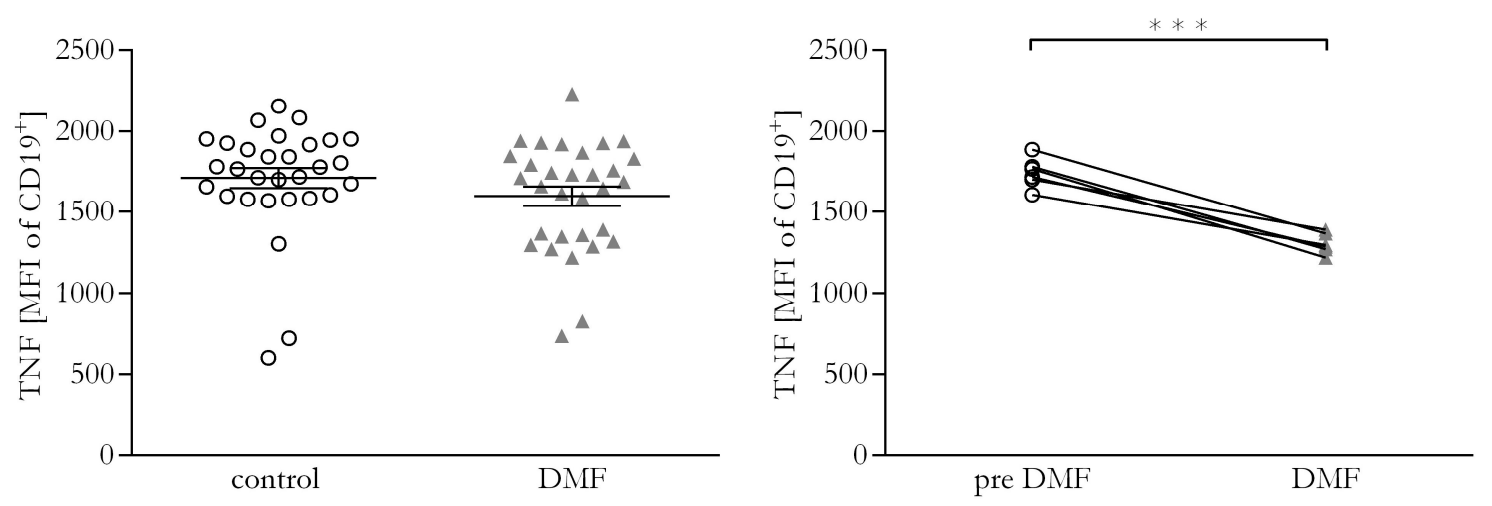

Figure 33: Tumor necrosis factor alpha production of $\mathrm{B}$ cells was decreased upon dimethyl fumarate treatment. Peripheral blood mononuclear cells were stimulated with $1 \mu \mathrm{g} / \mathrm{ml}$ cytosine-phosphate-guanine for 20 hours and $500 \mathrm{ng} / \mathrm{ml}$ ionomycin / $20 \mathrm{ng} / \mathrm{ml}$ phorbol 12-myristate 12 -acetate for 4 hours. The production of tumor necrosis factor alpha (TNF) by B cells $\left(\mathrm{CD} 19^{+}\right)$was quantified by flow cytometric intracellular staining given by the normalized mean fluorescent intensity $\left(\mathrm{MFI}_{\mathrm{n}}\right)$. In a cross-sectional setting, control multiple sclerosis patients (circles) were compared to dimethyl fumarate (DMF)-treated matches (triangles) (left graph). In addition, six patients' blood samples were taken before the initiation of DMF therapy (circles) and after at least 6 months of DMF treatment (triangles). Lines connect the values of individual patients. Bars indicate mean \pm standard error of the mean. ( $n=31$ (DMF); $n=30$ (control); $n=6$ (longitudinal); unpaired t-test (cross-sectional); Wilcoxon matched-pairs signed rank test (longitudinal); ${ }^{* * *}=\mathrm{p}<0.001$ )

\subsubsection{Production of anti-inflammatory IL-10 by B cells was slightly reduced upon DMF treatment}

In a next step, the ability of B cells to produce the anti-inflammatory cytokine IL-10 was analyzed. Again, cross-sectional as well as longitudinal data were collected. Figure 34 shows that DMF treatment significantly lowered the mean IL-10 intensity by $15.7 \%$ from an MFI of 345.6 to 291.5. The longitudinal samples showed the same trend but did not reach statistical significance.
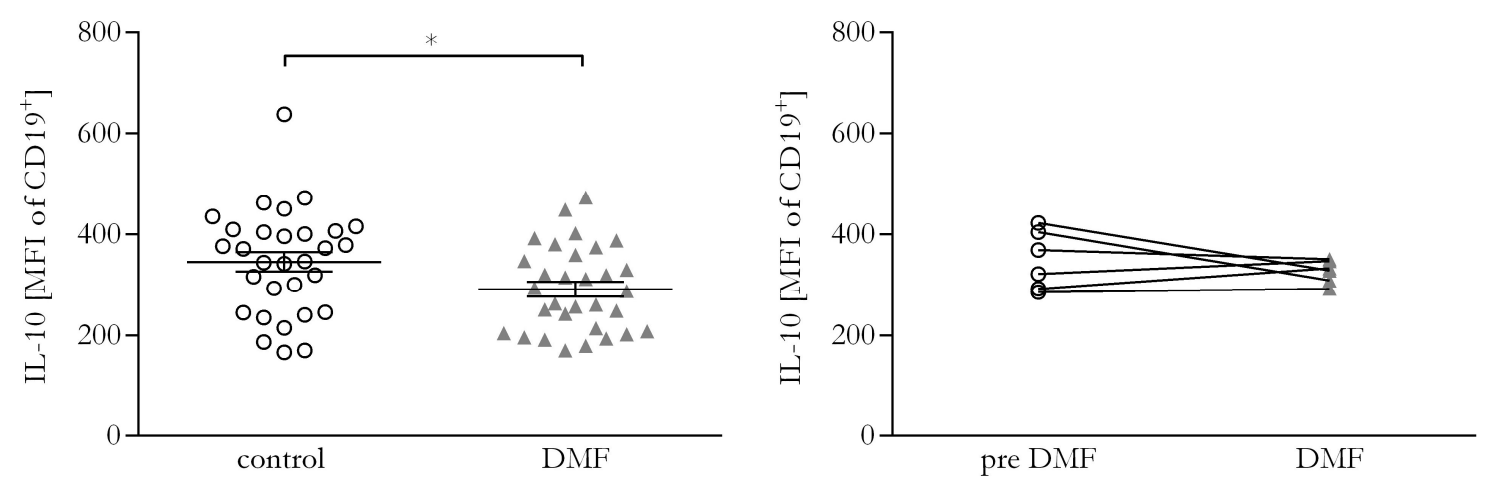

Figure 34: Dimethyl fumarate reduced the interleukin-10 production of B cells. Peripheral blood mononuclear cells were stimulated with $1 \mu \mathrm{g} / \mathrm{ml}$ cytosine-phosphate-guanine for 20 hours and $500 \mathrm{ng} / \mathrm{ml}$ ionomycin / $20 \mathrm{ng} / \mathrm{ml}$ phorbol 12-myristate 12-acetate for 4 hours. The production of interleukin-10 (IL-10) by B cells (CD19+) was quantified by flow cytometric intracellular staining given by the normalized mean fluorescent intensity $\left(\mathrm{MFI}_{\mathrm{n}}\right)$. In a cross-sectional setting, control multiple sclerosis patients (circles) were compared to dimethyl fumarate (DMF)-treated matches (triangles) (left graph). In addition, six patients' blood samples were taken before the initiation of DMF therapy (circles) and after at least 6 months of DMF treatment (triangles). Lines connect the values of individual patients. Bars indicate mean \pm standard error of the mean. ( $n=31$ (DMF); $n=30$ (control); $n=6$ (longitudinal); unpaired t-test (cross-sectional); Wilcoxon matched-pairs signed rank test (longitudinal); $*=p<0.05$ ) 


\subsubsection{DMF caused a shift towards a more anti-inflammatory cytokine profile in B cells}

The analysis of IL-6, TNF and IL-10 production by B cells revealed a decline in the synthesis of both pro- and anti-inflammatory cytokines upon DMF treatment. To evaluate whether this change is rather beneficial or harmful, the ratios of produced pro- and anti-inflammatory cytokines were calculated using the following formulas:

$$
\text { Anti inflammatory quotient }=\frac{M F I(I L ~ 10)}{M F I(I L 6)}=\frac{M F I(I L ~ 10)}{M F I(T N F)}
$$

As IL-10 is assumed to be anti-inflammatory and IL-6/TNF pro-inflammatory, an increase of this quotient represents a shift towards a more anti-inflammatory cytokine profile. Analyzing the cross-sectional data accordingly, no significant difference between both patient groups were found (Figure 35). However, looking at the longitudinal data, IL-10/IL-6 ratio increased indicating a more anti-inflammatory cytokine profile after DMF-treatment in individual patients.
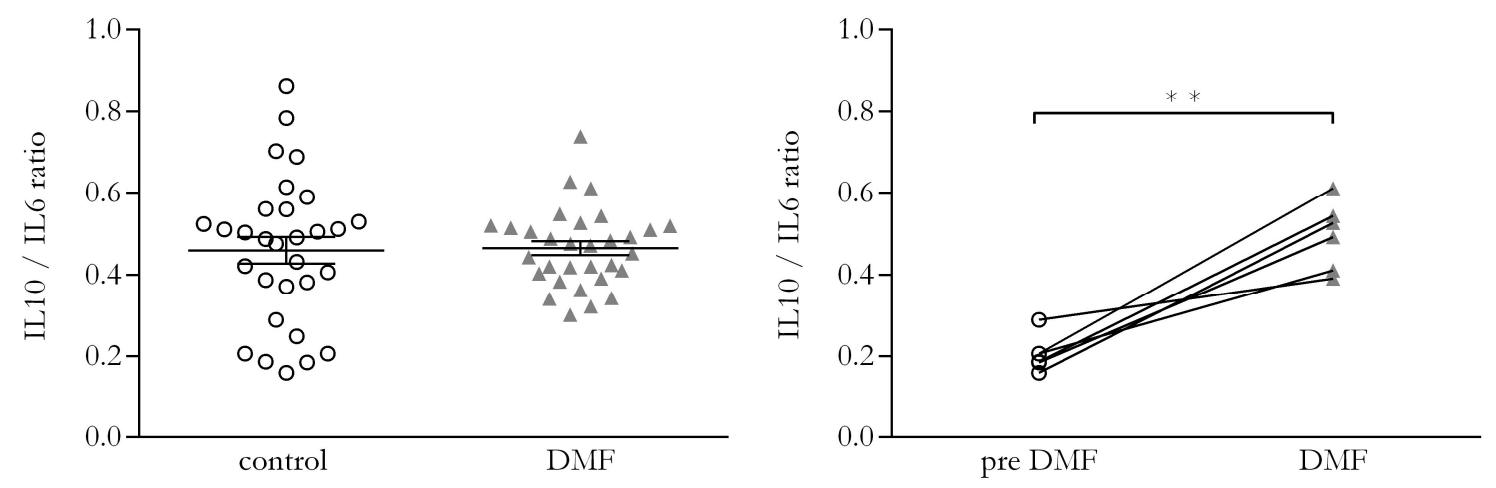

Figure 35: Interleukin-10 / interleukin-6 ratio was increased in B cells upon dimethyl fumarate treatment. Peripheral blood mononuclear cells were stimulated with $1 \mu \mathrm{g} / \mathrm{ml}$ cytosine-phosphate-guanine for 20 hours and $500 \mathrm{ng} / \mathrm{ml}$ ionomycin $/ 20$ $\mathrm{ng} / \mathrm{ml}$ phorbol 12-myristate 12-acetate for 4 hours. The production of interleukin-6 (IL-6) and interleukin-10 (IL-10) by B cells $\left(\mathrm{CD} 19^{+}\right)$was quantified by flow cytometric intracellular staining given by the normalized mean fluorescent intensity $\left(\mathrm{MFI}_{\mathrm{n}}\right)$ and the IL-10/IL-6 ratio was determined. In a cross-sectional setting, control multiple sclerosis patients (circles) were compared to dimethyl fumarate (DMF)-treated matches (triangles) (left graph). In addition, six patients' blood samples were taken before the initiation of DMF therapy (circles) and after at least 6 months of DMF treatment (triangles). Lines connect the values of individual patients. Bars indicate mean \pm standard error of the mean. $(n=31$ (DMF); $n=30$ (control); $\mathrm{n}=6$ (longitudinal); unpaired t-test (cross-sectional); Wilcoxon matched-pairs signed rank test (longitudinal); $* *=\mathrm{p}<0.01$ )

In parallel to the IL-10/IL-6 ratio, the IL-10/TNF ratio showed no significant difference when analyzing the cross-sectional data, but a significant anti-inflammatory shift in the longitudinally evaluated data of the individual patients (Figure 36). Table 16 summarizes the effect of DMF on the cytokine production of B cells. 

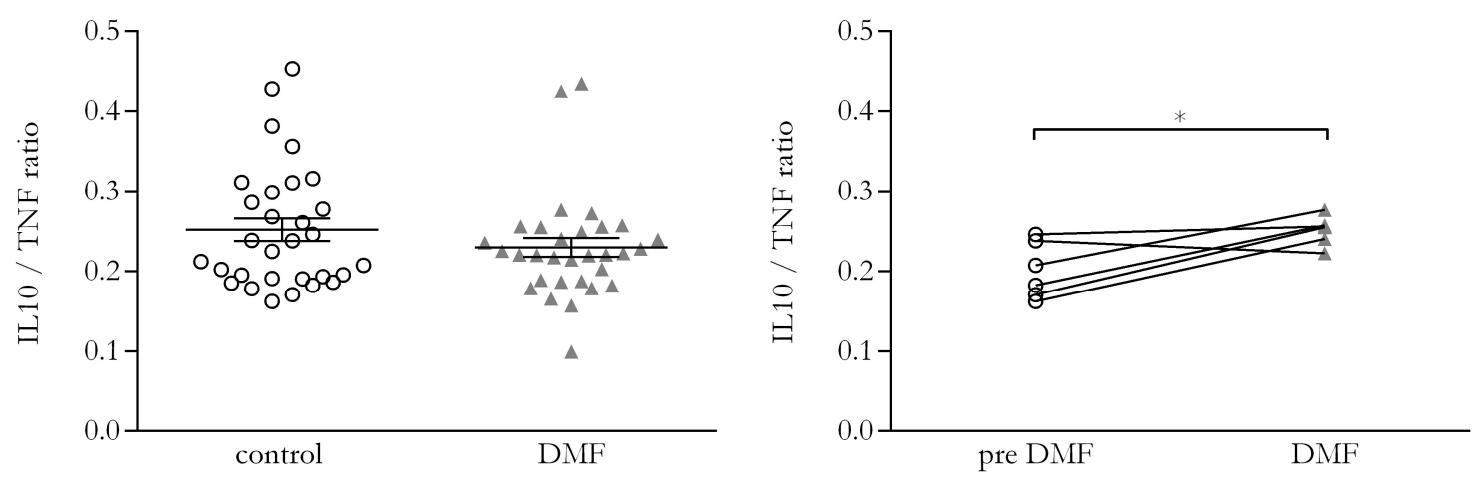

Figure 36: Dimethyl fumarate treatment elevated the interleukin-10 / tumor necrosis factor alpha ratio in B cells. Peripheral blood mononuclear cells were stimulated with $1 \mu \mathrm{g} / \mathrm{ml}$ cytosine-phosphate-guanine for 20 hours and $500 \mathrm{ng} / \mathrm{ml}$ ionomycin / $20 \mathrm{ng} / \mathrm{ml}$ phorbol 12-myristate 12-acetate for 4 hours. The production of tumor necrosis factor alpha (TNF) and interleukin-10 (IL-10) by B cells $\left(\mathrm{CD} 19^{+}\right)$was quantified by flow cytometric intracellular staining given by the normalized mean fluorescent intensity $\left(\mathrm{MFI}_{\mathrm{n}}\right)$ and the IL-10/TNF ratio was determined. In a cross-sectional setting, control multiple sclerosis patients (circles) were compared to dimethyl fumarate (DMF)-treated matches (triangles) (left graph). In addition, six patients' blood samples were taken before the initiation of DMF therapy (circles) and after at least 6 months of DMF treatment (triangles). Lines connect the values of individual patients. Bars indicate mean \pm standard error of the mean. ( $n=31$ (DMF); $n=30$ (control); $n=6$ (longitudinal); unpaired t-test (cross-sectional); Wilcoxon matched-pairs signed rank test (longitudinal); * $=\mathrm{p}<0.05$ )

Table 16: Dimethyl fumarate-induced changes of the cytokine production of B cells. $(\mathrm{SD}=$ standard deviation; $\mathrm{DMF}=$ dimethyl fumarate; $\mathrm{ns}=$ not significant $)$

\begin{tabular}{|l|c|c|c|c|c|}
\hline & $\begin{array}{c}\text { control } \\
(\mathrm{mean} \pm \mathbf{~ S D})\end{array}$ & $\begin{array}{c}\text { DMF-treated } \\
(\mathrm{mean} \pm \mathbf{~ S D})\end{array}$ & $\begin{array}{c}\text { percental } \\
\text { change }\end{array}$ & p value & \\
\hline Interleukin-6 & $1033 \pm 80.4$ & $788.1 \pm 41.7$ & $-23.7 \%$ & 0.0084 & $* *$ \\
Tumor necrosis factor & $1709 \pm 62.1$ & $1597 \pm 60.2$ & $-6.4 \%$ & 0.1973 & $\mathrm{~ns}$ \\
Interleukin-10 & $345.6 \pm 19.0$ & $291 \pm 15.0$ & $-15.7 \%$ & 0.0286 & $*$ \\
\hline
\end{tabular}

\subsubsection{In CD14 ${ }^{+}$myeloid cells, DMF inhibited pro-inflammatory IL-6 and TNF secretion}

Production of IL-6, TNF and IL-10 measured by intracellular cytokine staining was also obtained for $\mathrm{CD} 14^{+}$myeloid cells using flow cytometry. There was a significant reduction of IL-6 synthesis by $31.8 \%$ from a mean MFI of 3112 to 2122 in the cross-sectional samples upon DMF treatment. This trend was also seen in the longitudinal data, where a mean reduction by $43.9 \%$ was observed (Figure 37). In addition, a significantly reduced production of TNF was observed in $\mathrm{CD} 14^{+}$myeloid cells upon DMF. The MFI was decreased from a mean of 4072 in the control group to a mean MFI of 3562 in the DMF-treated group, equaling a reduction by $12.5 \%$. The longitudinal data showed the same, however not significant trend probably due to one outlier value (Figure 38). 

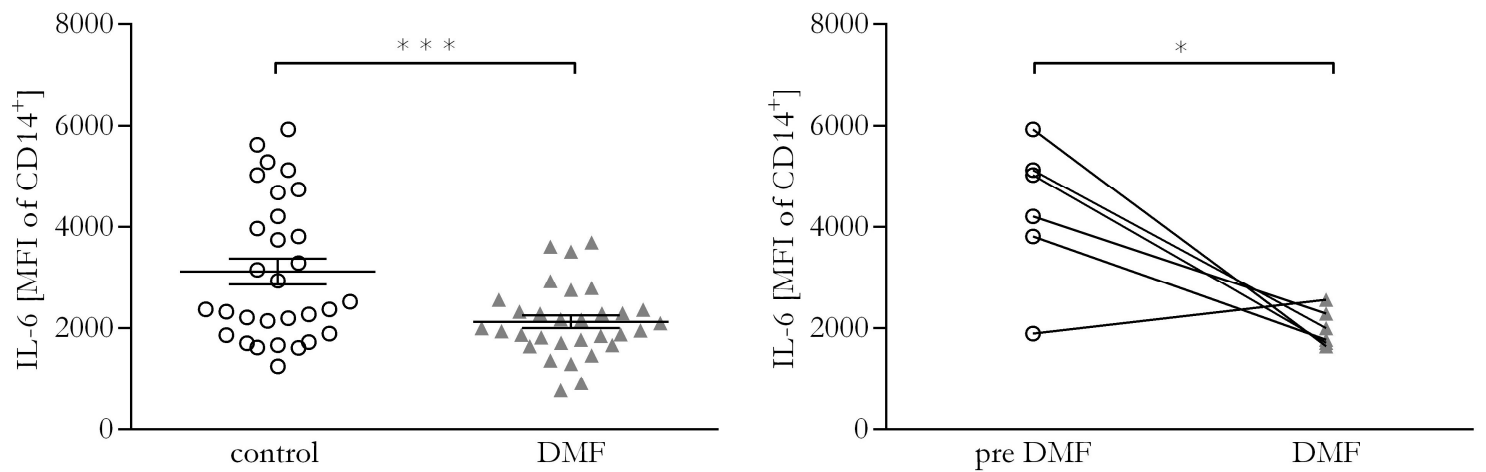

Figure 37: Interleukin-6 production in myeloid-derived phagocytes was reduced by dimethyl fumarate. Peripheral blood mononuclear cells were stimulated with $1 \mu \mathrm{g} / \mathrm{ml}$ cytosine-phosphate-guanine for 20 hours and $500 \mathrm{ng} / \mathrm{ml}$ ionomycin / 20 $\mathrm{ng} / \mathrm{ml}$ phorbol 12-myristate 12-acetate for 4 hours. The production of interleukin-6 (IL-6) by myeloid-derived phagocytes $\left(\mathrm{CD} 14^{+}\right)$was quantified by flow cytometric intracellular staining given by the normalized mean fluorescent intensity $\left(\mathrm{MFI}_{\mathrm{n}}\right)$. In a cross-sectional setting, control multiple sclerosis patients (circles) were compared to dimethyl fumarate (DMF)-treated matches (triangles) (left graph). In addition, six patients' blood samples were taken before the initiation of DMF therapy (circles) and after at least 6 months of DMF treatment (triangles). Lines connect the values of individual patients. Bars indicate mean \pm standard error of the mean. $(n=31$ (DMF); $n=30$ (control); $n=6$ (longitudinal); unpaired t-test (cross-sectional); Wilcoxon matched-pairs signed rank test (longitudinal); $*=p<0.05 ; * * *=p<0.001$ )
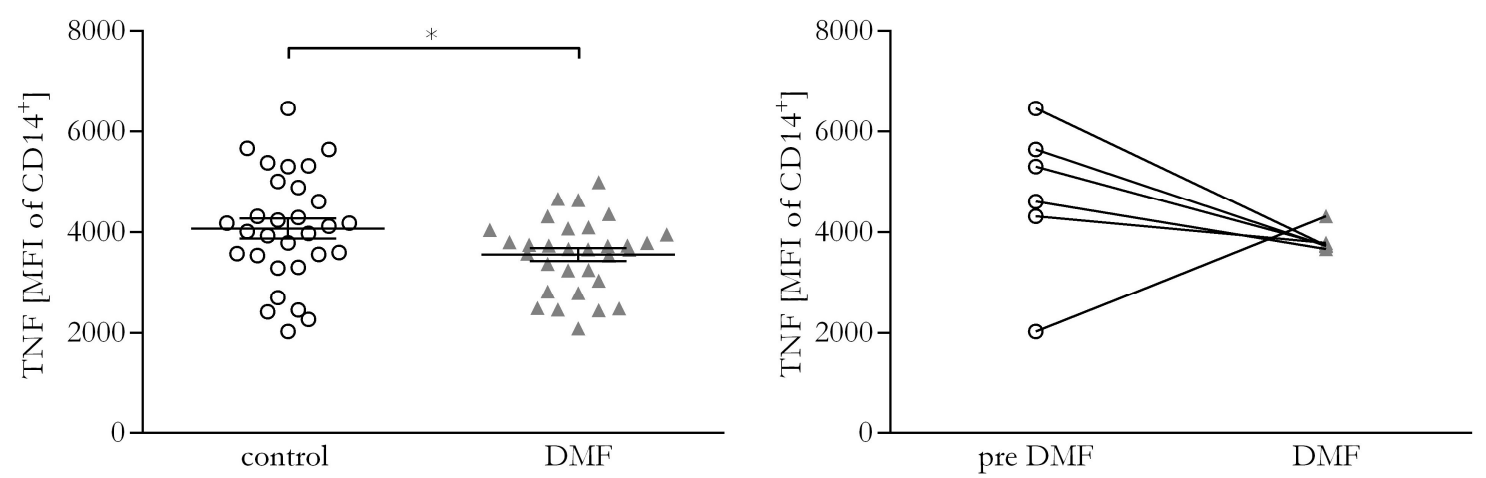

Figure 38: Tumor necrosis factor alpha production in $\mathrm{CD} 14^{+}$myeloid cells was reduced by dimethyl fumarate. Peripheral blood mononuclear cells were stimulated with $1 \mu \mathrm{g} / \mathrm{ml}$ cytosine-phosphate-guanine for 20 hours and $500 \mathrm{ng} / \mathrm{ml}$ ionomycin / $20 \mathrm{ng} / \mathrm{ml}$ phorbol 12-myristate 12 -acetate for 4 hours. The production of tumor necrosis factor alpha (TNF) by CD14+ myeloid cells was quantified by flow cytometric intracellular staining given by the normalized mean fluorescent intensity $\left(\mathrm{MFI}_{\mathrm{n}}\right)$. In a cross-sectional setting, control multiple sclerosis patients (circles) were compared to dimethyl fumarate (DMF)-treated matches (triangles) (left graph). In addition, six patients' blood samples were taken before the initiation of DMF therapy (circles) and after at least 6 months of DMF treatment (triangles). Lines connect the values of individual patients. Bars indicate mean \pm standard error of the mean. ( $n=31$ (DMF); $n=30$ (control); $n=6$ (longitudinal); unpaired t-test (cross-sectional); Wilcoxon matched-pairs signed rank test (longitudinal); *** $=\mathrm{p}<0.001$ )

\subsubsection{IL-10 production of CD14 ${ }^{+}$myeloid cells was not influenced by DMF}

The production of the anti-inflammatory cytokine IL-10 in myeloid-derived phagocytes was not significantly influenced by DMF treatment. Figure 39 shows that neither cross-sectional nor longitudinal data were significantly different upon treatment. 

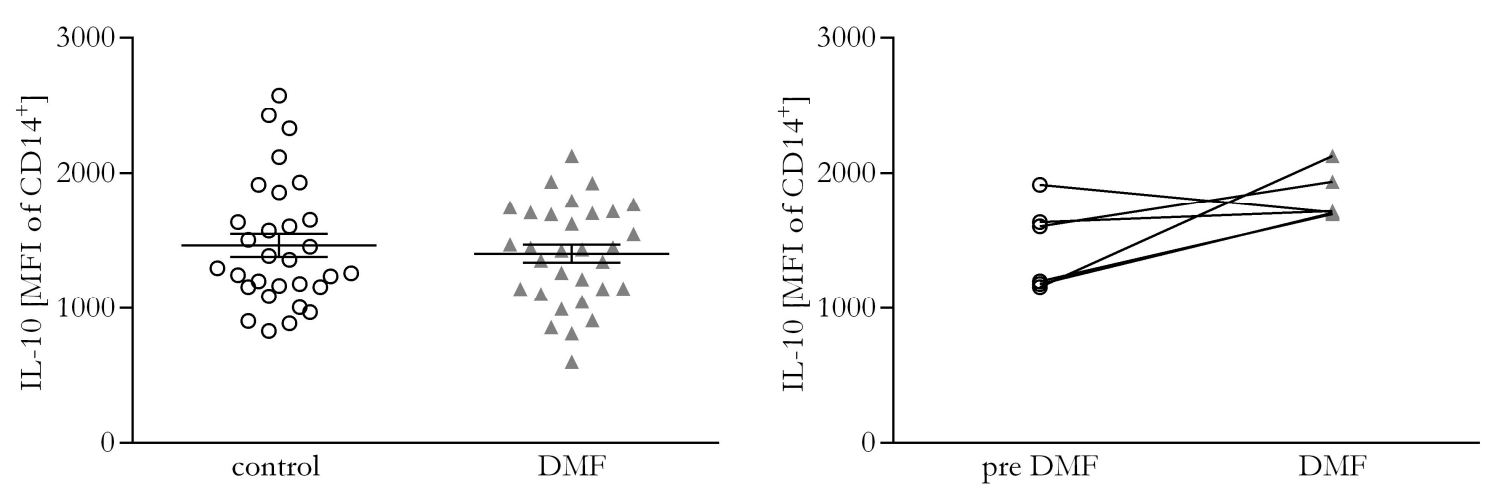

Figure 39: Dimethyl fumarate did not alter the interleukin-10 production of $\mathrm{CD} 14^{+}$myeloid cells. Peripheral blood mononuclear cells were stimulated with $1 \mu \mathrm{g} / \mathrm{ml}$ cytosine-phosphate-guanine for 20 hours and $500 \mathrm{ng} / \mathrm{ml}$ ionomycin / 20 $\mathrm{ng} / \mathrm{ml}$ phorbol 12-myristate 12 -acetate for 4 hours. The production of interleukin-10 (IL-10) by CD14+ myeloid cells was quantified by flow cytometric intracellular staining given by the normalized mean fluorescent intensity $\left(\mathrm{MFI}_{\mathrm{n}}\right)$. In a crosssectional setting, control multiple sclerosis patients (circles) were compared to dimethyl fumarate (DMF)-treated matches (triangles) (left graph). In addition, six patients' blood samples were taken before the initiation of DMF therapy (circles) and after at least 6 months of DMF treatment (triangles). Lines connect the values of individual patients. Bars indicate mean \pm standard error of the mean. ( $n=31$ (DMF); $n=30$ (control); $n=6$ (longitudinal); unpaired t-test (cross-sectional); Wilcoxon matched-pairs signed rank test (longitudinal); not significant)

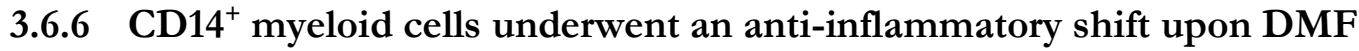 treatment}

To classify the effects of DMF on the cytokine production of myeloid-derived phagocytes in a more functional way, the same anti-inflammatory quotients as for B cells were calculated. A significant change in the IL-10/IL-6 ratio was seen both in cross-sectional and longitudinal data towards a more anti-inflammatory cytokine response profile. The quotient IL-10/TNF revealed the same trend but was not significant (Figures 40+41).
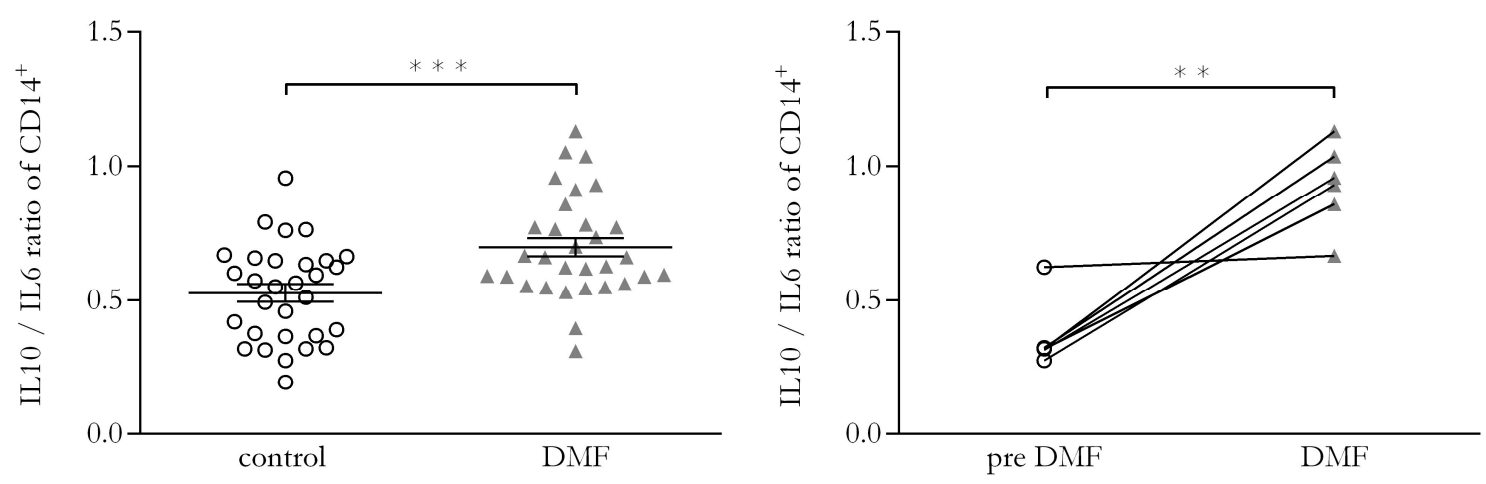

Figure 40: Interleukin-10 / interleukin-6 ratio increased in CD14+ myeloid cells upon dimethyl fumarate. Peripheral blood mononuclear cells were stimulated with $1 \mu \mathrm{g} / \mathrm{ml}$ cytosine-phosphate-guanine for 20 hours and $500 \mathrm{ng} / \mathrm{ml}$ ionomycin $/ 20$ $\mathrm{ng} / \mathrm{ml}$ phorbol 12-myristate 12-acetate for 4 hours. The production of interleukin-6 (IL-6) and interleukin-10 (IL-10) by $\mathrm{CD} 14^{+}$myeloid cells was quantified by flow cytometric intracellular staining given by the normalized mean fluorescent intensity $\left(\mathrm{MFI}_{\mathrm{n}}\right)$ and the IL-10/IL-6 ratio was determined. In a cross-sectional setting, control multiple sclerosis patients (circles) were compared to dimethyl fumarate (DMF)-treated matches (triangles) (left graph). In addition, six patients' blood samples were taken before the initiation of DMF therapy (circles) and after at least 6 months of DMF treatment (triangles). Lines connect the values of individual patients. Bars indicate mean \pm standard error of the mean. $(n=31$ (DMF); $n=30$ (control); $n=6$ (longitudinal); unpaired t-test (cross-sectional); Wilcoxon matched-pairs signed rank test (longitudinal); ** $=\mathrm{p}<0.01 ; * * *=\mathrm{p}<0.001)$ 

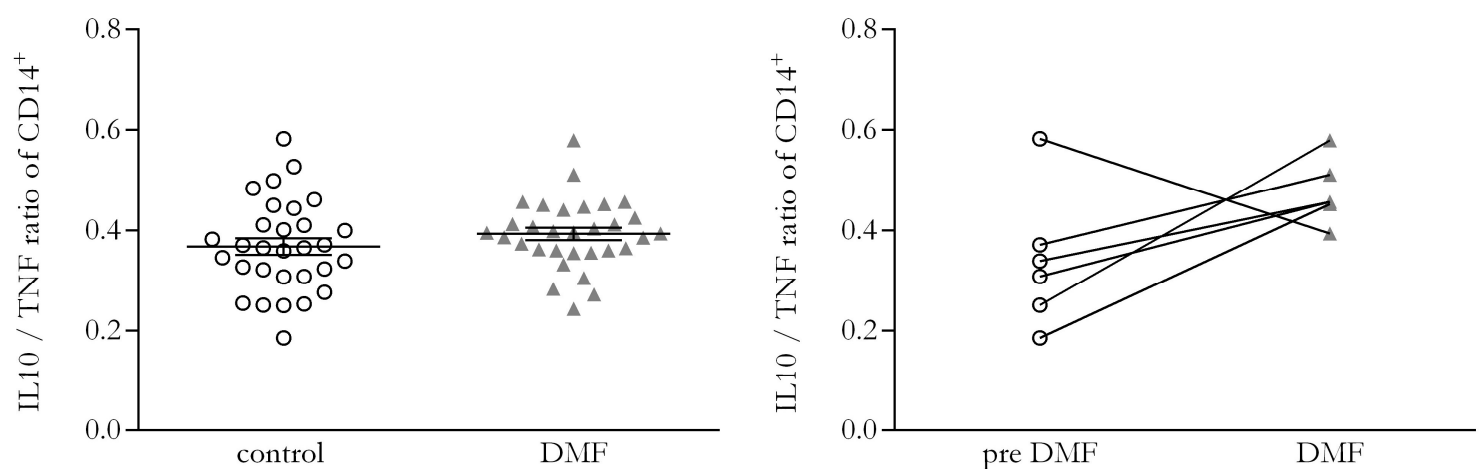

Figure 41: In $\mathrm{CD} 14^{+}$myeloid cells, the interleukin-10 / tumor necrosis factor ratio was not altered by dimethyl fumarate. Peripheral blood mononuclear cells were stimulated with $1 \mu \mathrm{g} / \mathrm{ml}$ cytosine-phosphate-guanine for 20 hours and $500 \mathrm{ng} / \mathrm{ml}$ ionomycin / $20 \mathrm{ng} / \mathrm{ml}$ phorbol 12-myristate 12 -acetate for 4 hours. The production of tumor necrosis factor alpha (TNF) and interleukin-10 (IL-10) by CD14+ myeloid cells was quantified by flow cytometric intracellular staining given by the normalized mean fluorescent intensity $\left(\mathrm{MFI}_{\mathrm{n}}\right)$ and the IL-10/TNF ratio was determined. In a cross-sectional setting, control multiple sclerosis patients (circles) were compared to dimethyl fumarate (DMF)-treated matches (triangles) (left graph). In addition, six patients' blood samples were taken before the initiation of DMF therapy (circles) and after at least 6 months of DMF treatment (triangles). Lines connect the values of individual patients. Bars indicate mean \pm standard error of the mean. ( $n=31$ (DMF); $n=30$ (control); $n=6$ (longitudinal); unpaired t-test (cross-sectional); Wilcoxon matched-pairs signed rank test (longitudinal); not significant)

Table 17: Dimethyl fumarate-induced changes of the cytokine production of $\mathrm{CD} 14^{+}$myeloid cells. $(\mathrm{SD}=$ standard deviation; $\mathrm{DMF}=$ dimethyl fumarate $)$

\begin{tabular}{|l|c|c|c|c|c|}
\hline & $\begin{array}{c}\text { control } \\
(\mathrm{mean} \pm \mathbf{S D})\end{array}$ & $\begin{array}{c}\text { DMF-treated } \\
(\mathrm{mean} \pm \mathbf{S D})\end{array}$ & $\begin{array}{c}\text { percental } \\
\text { change }\end{array}$ & p value & \\
\hline Interleukin-6 & $3112 \pm 256.5$ & $2122 \pm 125.0$ & $-31.8 \%$ & 0.0009 & $* * *$ \\
Tumor necrosis factor & $4072 \pm 198.9$ & $3562 \pm 128.6$ & $-12.5 \%$ & 0.0343 & $*$ \\
Interleukin-10 & $1465 \pm 84.96$ & $1404 \pm 66.44$ & $-4.2 \%$ & 0.5687 & $\mathrm{~ns}$ \\
\hline
\end{tabular}

\subsubsection{The ELISA of supernatants confirmed a trend towards anti-inflammation}

The results of the intracellular staining uniformly showed a shift towards a more anti-inflammatory cytokine profile in both $\mathrm{B}$ cells and $\mathrm{CD} 14^{+}$myeloid cells. To reinforce these results, the concentration of secreted cytokines was determined in the supernatant of these cells using ELISA. The respective supernatants were harvested after stimulation with CpG for 20 hours in parallel to the intracellular flow cytometry stainings.

The concentration of IL- 6 and IL-10 was measures in the supernatant by ELISA using the standard protocol as described above. No significant changes were found in the levels of IL-6 or IL-10 in the supernatants, neither in the cross-sectional analysis nor in the longitudinal samples (Figures 42+43). 

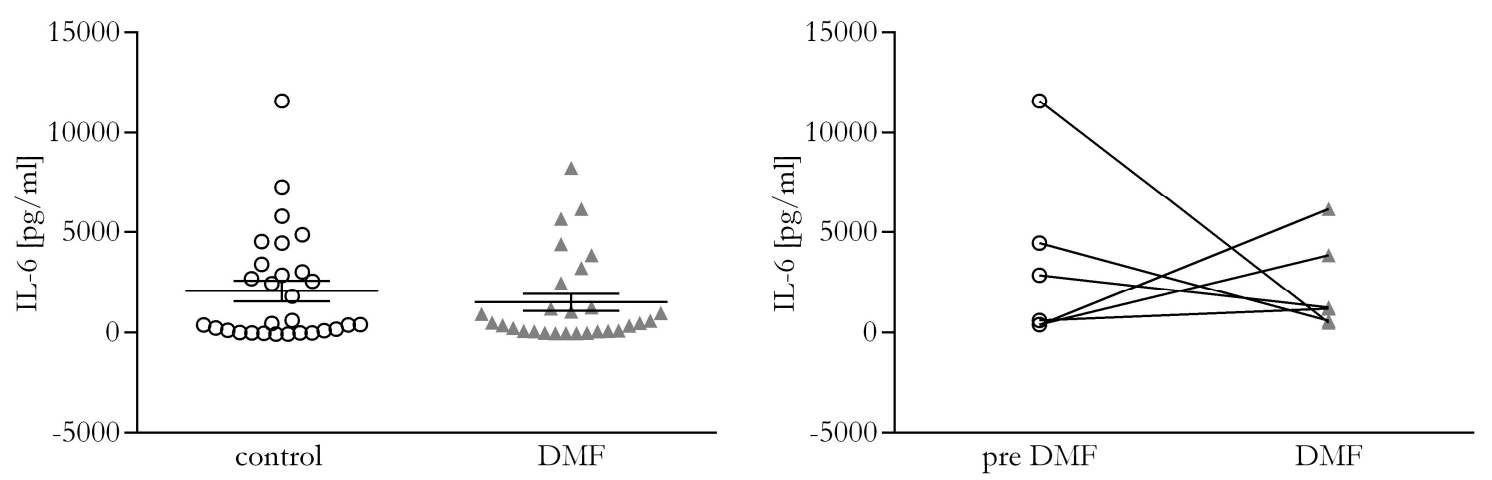

Figure 42: The interleukin-6 concentration was not changed in the supernatant. Peripheral blood mononuclear cells were stimulated with $1 \mu \mathrm{g} / \mathrm{ml}$ cytosine-phosphate-guanine for 20 hours and $500 \mathrm{ng} / \mathrm{ml}$ ionomycin $/ 20 \mathrm{ng} / \mathrm{ml}$ phorbol 12-myristate 12-acetate for 4 hours. Enzyme linked immunosorbent assay was performed to measure the concentration of interleukin-6 (IL-6) in the supernatant. In a cross-sectional setting, control multiple sclerosis patients (circles) were compared to dimethyl fumarate (DMF)-treated matches (triangles) (left graph). In addition, six patients' blood samples were taken before the initiation of DMF therapy (circles) and after at least 6 months of DMF treatment (triangles). Lines connect the values of individual patients. Bars indicate mean \pm standard error of the mean. $(n=31$ (DMF); $n=30$ (control); $n=6$ (longitudinal); unpaired t-test (cross-sectional); Wilcoxon matched-pairs signed rank test (longitudinal); not significant)
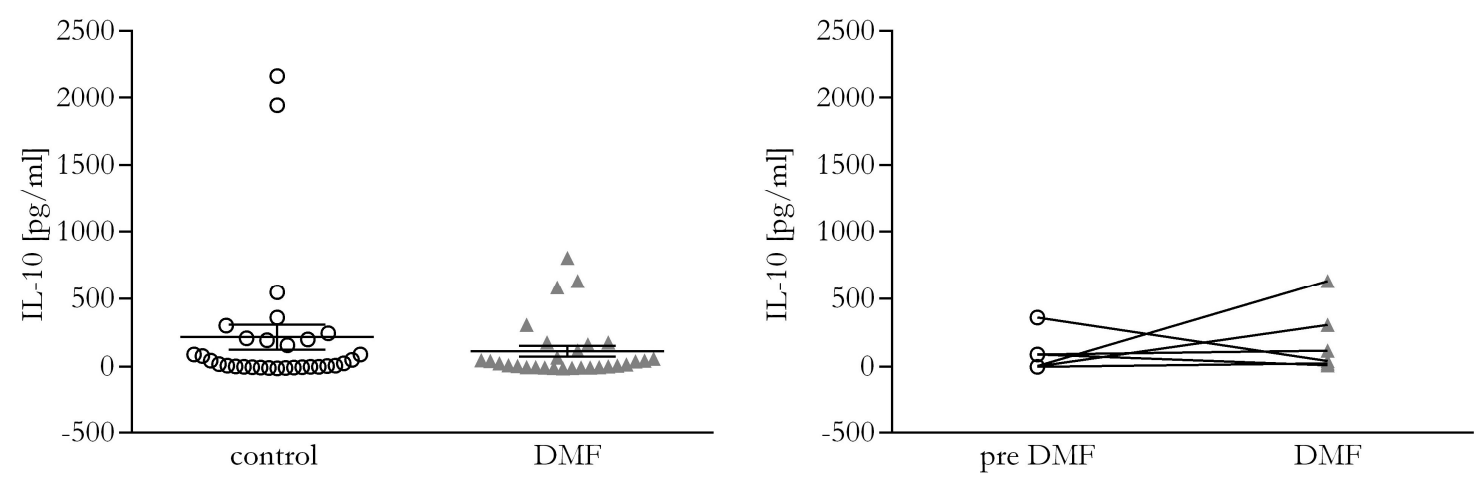

Figure 43: Dimethyl fumarate does not change the concentration of interleukin-10 in the supernatant. Peripheral blood mononuclear cells were stimulated with $1 \mu \mathrm{g} / \mathrm{ml}$ cytosine-phosphate-guanine for 20 hours and $500 \mathrm{ng} / \mathrm{ml}$ ionomycin / $20 \mathrm{ng} / \mathrm{ml}$ phorbol 12-myristate 12-acetate for 4 hours. Enzyme linked immunosorbent assay was performed to measure the concentration of interleukin-10 (IL-10) in the supernatant. In a cross-sectional setting, control multiple sclerosis patients (circles) were compared to dimethyl fumarate (DMF)-treated matches (triangles) (left graph). In addition, six patients' blood samples were taken before the initiation of DMF therapy (circles) and after at least 6 months of DMF treatment (triangles). Lines connect the values of individual patients. Bars indicate mean \pm standard error of the mean. $(n=31$ (DMF); $n=30$ (control); $n=6$ (longitudinal); unpaired t-test (cross-sectional); Wilcoxon matched-pairs signed rank test (longitudinal); not significant)

However, when the anti-inflammatory quotient was calculated analogous to the intracellular cytokine staining (see formula below), a significant increase of this quotient was seen in the longitudinal samples and a similar trend in the cross-sectional ones. This confirms the shift towards an anti-inflammatory cytokine profile observed by flow cytometry (Figure 44).

$$
\text { Anti inflammatory quotient }=\frac{c(I L 10)}{c(I L 6)}
$$



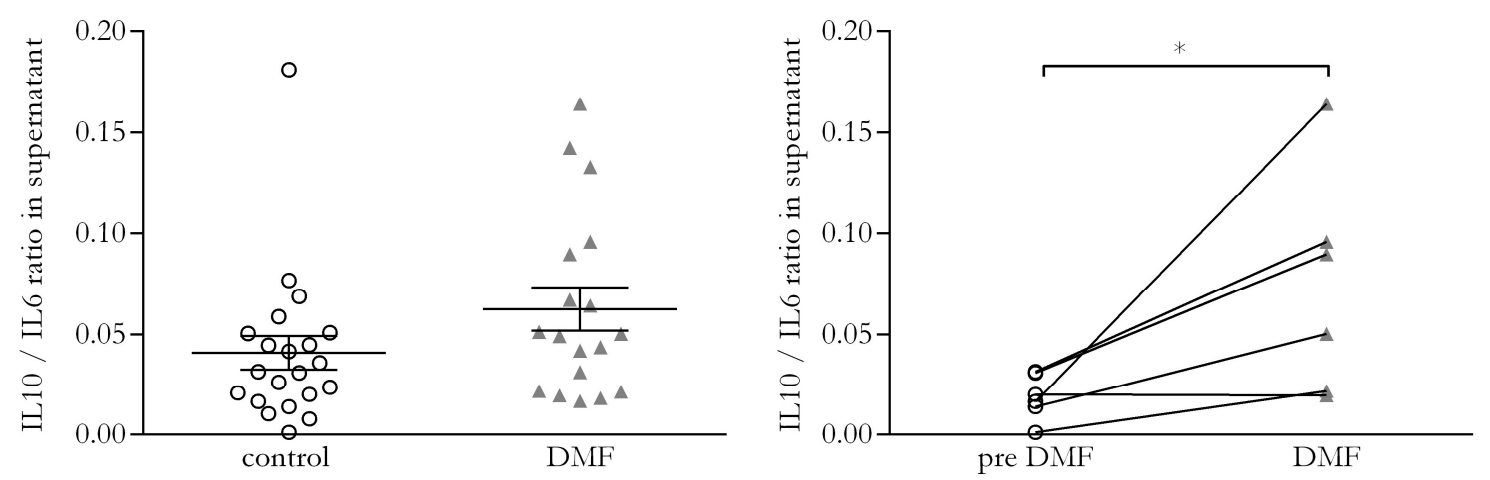

Figure 44: Dimethyl fumarate induced an anti-inflammatory shift in the supernatant. Peripheral blood mononuclear cells were stimulated with $1 \mu \mathrm{g} / \mathrm{ml}$ cytosine-phosphate-guanine for 20 hours and $500 \mathrm{ng} / \mathrm{ml}$ ionomycin $/ 20 \mathrm{ng} / \mathrm{ml}$ phorbol 12myristate 12 -acetate for 4 hours. Enzyme linked immunosorbent assay was performed to measure the concentration of interleukin-6 (IL-6) and interleukin-10 (IL-10) in the supernatant and the IL-10/IL-6 ratio was determined. In a crosssectional setting, control multiple sclerosis patients (circles) were compared to dimethyl fumarate (DMF)-treated matches (triangles) (left graph). In addition, six patients' blood samples were taken before the initiation of DMF therapy (circles) and after at least 3 months of DMF treatment (triangles). Lines connect the values of individual patients. Bars indicate mean \pm standard error of the mean. ( $n=31$ (DMF); $n=30$ (control); $n=6$ (longitudinal); unpaired t-test (cross-sectional); Wilcoxon matched-pairs signed rank test (longitudinal); $*=\mathrm{p}<0.05)$

\subsection{The influence of treatment duration on DMF-mediated changes in the immune cell compartment}

Next, this examination aimed to evaluate, if the observed changes in the immune cell compartments upon DMF treatment were transitional, stable over time or intensifying with prolonged DMF treatment. Therefore, correlation studies determining the Pearson correlation coefficient $(r)$ and coefficient of determination $\left(r^{2}\right)$ for treatment duration and cell distribution, expression of surface marker and cytokine production were performed.

\subsubsection{Changes in the immune cell distribution were stable during prolonged DMF treatment}

First, treatment duration was correlated with the immune cell frequencies obtained by flow cytometry. As shown in Table 18, there were trends, but no significant changes most probably due to the small size of the patient cohort. Figure 45 exemplarily displays the correlations of $\mathrm{CD} 14^{+}$myeloid cells and $\mathrm{CD}^{+} \mathrm{T}$ cells.

Table 18: Correlation of cell frequencies with duration of dimethyl fumarate medication. $\left(\mathrm{r}^{2}=\right.$ coefficient of determination; $r=$ Pearson correlation coefficient)

\begin{tabular}{|l|c|c|c|c|c|c|}
\hline & unit & $\begin{array}{c}\text { slope } \\
\text { (units/month) }\end{array}$ & $\mathbf{r}^{\mathbf{2}}$ & $\mathbf{r}$ & p value & \\
\hline B cells & $\%$ & 0.009311 & 0.0003 & 0.0176 & 0.9129 & $\mathrm{~ns}$ \\
CD14 ${ }^{+}$cells & $\%$ & 0.158 & 0.2549 & 0.5049 & 0.3187 & $\mathrm{~ns}$ \\
CD4 $^{+}$T cells & $\%$ & 0.2294 & 0.2744 & 0.5238 & 0.3731 & $\mathrm{~ns}$ \\
CD8 $^{+}$T cells & $\%$ & -0.08262 & 0.0007 & 0.0269 & 0.6487 & $\mathrm{~ns}$ \\
\hline
\end{tabular}



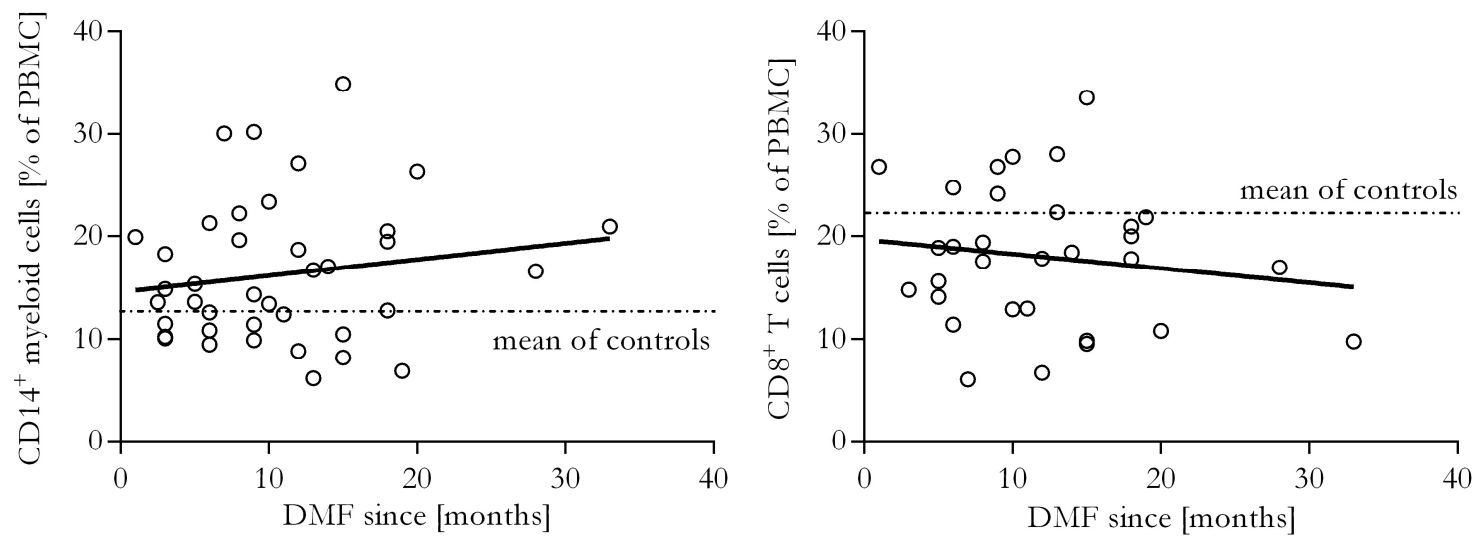

Figure 45: $\mathrm{CD}_{14}+$ myeloid cell and $\mathrm{CD} 8^{+} \mathrm{T}$ cell frequencies as a function of dimethyl fumarate treatment duration. The frequencies of $\mathrm{CD}_{14}+$ myeloid cells and cytotoxic $\mathrm{T}$ cells $(\mathrm{CD} 8+)$ within the peripheral blood mononuclear cells (PBMC) were determined by flow cytometry. The individual patients' values (dimethyl fumarate (DMF)-treated patients are represented by circles) were correlated with the time in months they had taken DMF using linear regression. $(n=31$ (DMF); $\left(\mathrm{CD} 14^{+}\right.$myeloid cells: Pearson correlation coefficient $\left.(\mathrm{r})=0.5049 ; \mathrm{p}=0.3187\right)\left(\mathrm{CD}^{+} \mathrm{T}\right.$ cells: $\left.\mathrm{r}=0.0269 ; \mathrm{p}=0.6487\right)$

\subsubsection{No long-term changes in $B$ cell subsets}

The observed changes in the B cell subsets were stable over time as there was no additional change in their frequency with prolonged DMF treatment (Table 19).

Table 19: Correlation between $B$ cell subset frequency and the duration of dimethyl fumarate intake. $\left(r^{2}=\right.$ coefficient of determination; $r=$ Pearson correlation coefficient; $\mathrm{Ag}=$ antigen; $\mathrm{BC}=\mathrm{B}$ cells $)$

\begin{tabular}{|l|c|c|c|c|c|c|}
\hline & unit & $\begin{array}{c}\text { slope } \\
\text { (units/month) }\end{array}$ & $\mathbf{r}^{2}$ & $\mathbf{r}$ & p value & \\
\hline Transitional B cells & $\%$ & 0.1034 & 0.0181 & 0.1346 & 0.4705 & $\mathrm{~ns}$ \\
Mature B cells & $\%$ & -0.05449 & 0.0065 & 0.0804 & 0.6672 & $\mathrm{~ns}$ \\
Ag-experienced BC & $\%$ & -0.1164 & 0.0211 & 0.1451 & 0.4361 & $\mathrm{~ns}$ \\
Memory B cells & $\%$ & -0.217 & 0.0278 & 0.1669 & 0.3697 & $\mathrm{~ns}$ \\
Plasmablasts & $\%$ & -0.04216 & 0.0484 & 0.2201 & 0.2342 & $\mathrm{~ns}$ \\
\hline
\end{tabular}

\subsubsection{Activation markers continued to decrease with DMF treatment duration}

Next, it was investigated whether the duration of DMF treatment correlates with its inhibitory effect on activation marker on B cells and myeloid cells. Most investigated activation markers showed a trend towards a lower expression on professional APC with prolonged DMF treatment, indicated by a negative correlation slope. However, most of them did not reach statistical significance (Table 20). Of all analyzed parameter, only $\mathrm{CD} 69^{+} \mathrm{B}$ cell frequency and $\mathrm{CD} 80^{+} \mathrm{B}$ cell frequency correlated negatively with the duration of DMF intake. This may indicate that the longer patients had taken DMF, the stronger was the inhibitory effect on B cell activation (Figure 46). In contrast, MHC-II expression on myeloid-derived phagocytes was correlating positively with the duration of DMF intake as shown above in Figure 30. 
Table 20: Correlation between cell surface markers and the duration of dimethyl fumarate intake. $\left(\mathrm{r}^{2}\right.$ $=$ coefficient of determination; $\mathrm{r}=$ Pearson correlation coefficient $\mathrm{BC}=\mathrm{B}$ cells; $\mathrm{CD}=$ cluster of differentiation; $\mathrm{CPG}=$ cytosine-phosphate-guanine; $\mathrm{MFI}=$ mean fluorescent intensity; $\mathrm{MHC}-\mathrm{II}=$ major histocompatibility complex - class II; MC = myeloid cells; LPS = lipopolysaccharides; ns = not significant)

\begin{tabular}{|c|c|c|c|c|c|c|c|}
\hline & stimulation & unit & $\begin{array}{c}\text { slope } \\
\text { (units/month) }\end{array}$ & $\mathbf{r}^{2}$ & $\mathbf{r}$ & $\underset{\text { value }}{\mathrm{p}}$ & \\
\hline \multicolumn{8}{|c|}{ B cells $\left.\left(\mathrm{BC} ; \mathrm{CD}^{\prime}\right)^{+}\right)$} \\
\hline $\mathrm{CD}_{25}+\mathrm{BC}$ & $2 \mu \mathrm{g} / \mathrm{ml} \mathrm{CpG}$ & $\%$ & -0.1249 & 0.0184 & 0.1355 & 0.4833 & $\mathrm{~ns}$ \\
\hline CD25 & $2 \mu \mathrm{g} / \mathrm{ml} \mathrm{CpG}$ & MFI & 5.792 & 0.0235 & 0.1531 & 0.4278 & ns \\
\hline CD40 & unstimulated & MFI & -11.42 & 0.0282 & 0.1678 & 0.2942 & ns \\
\hline CD40 & $2 \mu \mathrm{g} / \mathrm{ml} \mathrm{CpG}$ & MFI & 19.57 & 0.0113 & 0.1063 & 0.6208 & $\mathrm{~ns}$ \\
\hline $\mathrm{CD} 69^{+} \mathrm{BC}$ & $2 \mu \mathrm{g} / \mathrm{ml} \mathrm{CpG}$ & $\%$ & -0.5697 & 0.1335 & 0.3654 & 0.0433 & $*$ \\
\hline CD69 & $2 \mu \mathrm{g} / \mathrm{ml} \mathrm{CpG}$ & MFI & -10.52 & 0.1008 & 0.3175 & 0.0817 & ns \\
\hline $\mathrm{CD}^{8} 0^{+} \mathrm{BC}$ & unstimulated & $\%$ & -0.2324 & 0.1041 & 0.3226 & 0.0452 & $*$ \\
\hline $\mathrm{CD}^{2} 0^{+} \mathrm{BC}$ & $2 \mu \mathrm{g} / \mathrm{ml} \mathrm{CpG}$ & $\%$ & -0.1749 & 0.0378 & 0.1944 & 0.2948 & ns \\
\hline CD80 & unstimulated & MFI & -1.662 & 0.0890 & 0.2983 & 0.0651 & ns \\
\hline CD80 & $2 \mu \mathrm{g} / \mathrm{ml} \mathrm{CpG}$ & MFI & -2.733 & 0.0103 & 0.1015 & 0.5869 & ns \\
\hline $\mathrm{CD}^{2} 6^{+} \mathrm{BC}$ & $2 \mu \mathrm{g} / \mathrm{ml} \mathrm{CpG}$ & $\%$ & -0.2832 & 0.0944 & 0.3072 & 0.0927 & ns \\
\hline CD86 & unstimulated & MFI & 0.1476 & 0.0010 & 0.0317 & 0.8439 & ns \\
\hline CD86 & $2 \mu \mathrm{g} / \mathrm{ml} \mathrm{CpG}$ & MFI & -4.985 & 0.0347 & 0.1861 & 0.316 & ns \\
\hline $\mathrm{CD} 95^{+} \mathrm{BC}$ & unstimulated & $\%$ & -0.2015 & 0.0981 & 0.3133 & 0.0522 & $\mathrm{~ns}$ \\
\hline CD95 & unstimulated & MFI & -5.397 & 0.0191 & 0.1382 & 0.4016 & ns \\
\hline CD95 & $2 \mu \mathrm{g} / \mathrm{ml} \mathrm{CpG}$ & MFI & -34.63 & 0.0305 & 0.1745 & 0.3478 & ns \\
\hline CD150 & unstimulated & MFI & -0.2155 & 0.0441 & 0.2100 & 0.2834 & ns \\
\hline CD150 & $100 \mathrm{pg} / \mathrm{ml} \mathrm{LPS}$ & MFI & -0.2101 & 0.0527 & 0.2297 & 0.2139 & ns \\
\hline MHC-II & unstimulated & MFI & 110.4 & 0.0555 & 0.2356 & 0.1381 & ns \\
\hline MHC-II & $2 \mu \mathrm{g} / \mathrm{ml} \mathrm{CpG}$ & MFI & -54.477 & 0.0085 & 0.0920 & 0.6226 & ns \\
\hline \multicolumn{8}{|c|}{ 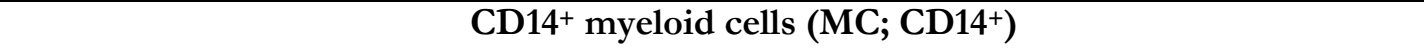 } \\
\hline CD40 & unstimulated & MFI & 0.2945 & 0.0001 & 0.0072 & 0.9643 & $\mathrm{~ns}$ \\
\hline $\mathrm{CD}^{2}{ }^{+} \mathrm{MC}$ & unstimulated & $\%$ & 0.07686 & 0.0066 & 0.0812 & 0.6136 & ns \\
\hline CD80 & unstimulated & MFI & 0.2905 & 0.0017 & 0.0407 & 0.8005 & $\mathrm{~ns}$ \\
\hline CD86 & unstimulated & MFI & 0.426 & 0.0001 & 0.0114 & 0.9434 & ns \\
\hline CD95 & unstimulated & MFI & 29.92 & 0.0451 & 0.2124 & 0.1824 & ns \\
\hline $\mathrm{CD} 150^{+} \mathrm{MC}$ & unstimulated & $\%$ & -0.541 & 0.0331 & 0.1818 & 0.3544 & ns \\
\hline $\mathrm{CD} 150^{+} \mathrm{MC}$ & $100 \mathrm{pg} / \mathrm{ml} \mathrm{LPS}$ & $\%$ & -0.4572 & 0.0179 & 0.1339 & 0.4725 & ns \\
\hline MHC-II & unstimulated & MFI & 149.3 & 0.1170 & 0.3421 & 0.0286 & $*$ \\
\hline MHC-II & $100 \mathrm{pg} / \mathrm{ml} \mathrm{LPS}$ & MFI & -494.8 & 0.0447 & 0.2113 & 0.2538 & ns \\
\hline
\end{tabular}



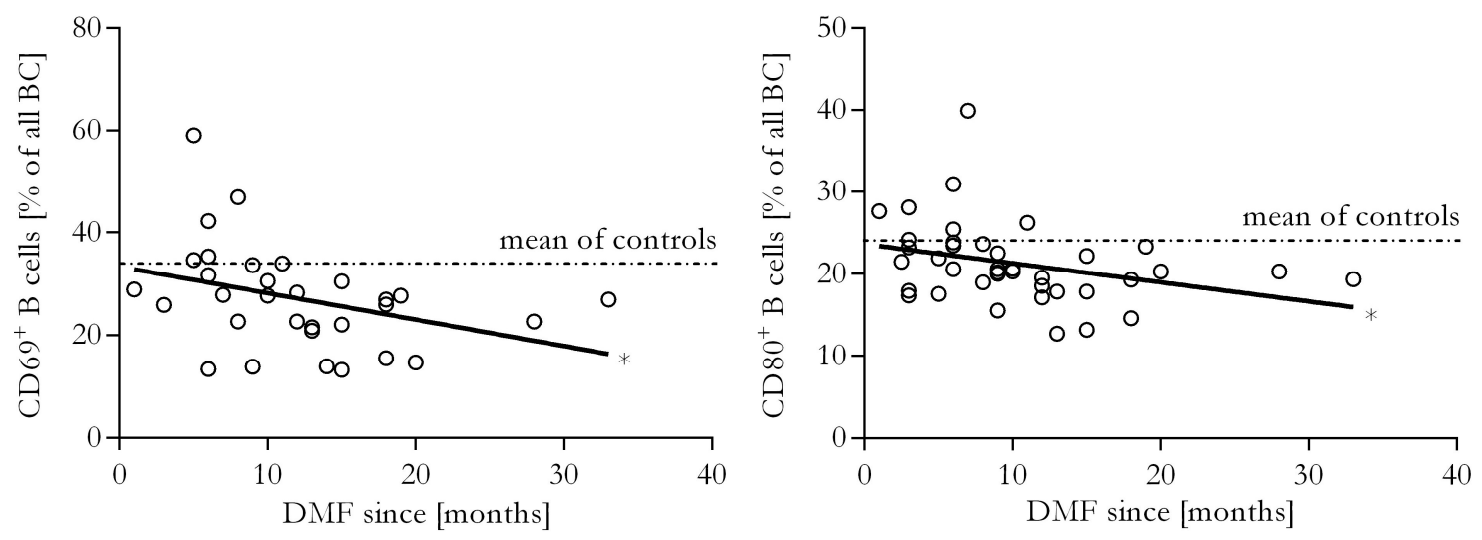

Figure 46: $\mathrm{CD} 69^{+}$and $\mathrm{CD} 80^{+} \mathrm{B}$ cell frequencies as a function of dimethyl fumarate treatment duration. The frequencies of $\mathrm{CD} 69^{+}$and $\mathrm{CD} 80^{+} \mathrm{B}$ cells $\left(\mathrm{BC}\right.$; $\mathrm{CD} 19^{+}$) within the peripheral blood mononuclear cells (PBMC) were determined by flow cytometry. The individual patients' values (dimethyl fumarate (DMF)-treated patients are represented by circles) were correlated with the time in months they had taken DMF using linear regression $\left(n=31\right.$ (DMF); CD69 ${ }^{+} \mathrm{BC}$ : Pearson correlation coefficient $\left.(r)=0.3654 ; \mathrm{p}=0.0433 ; \mathrm{CD} 80^{+} \mathrm{BC}: \mathrm{r}=0.3226 ; \mathrm{p}=0.0452\right)$.

\subsection{Epidemiological analysis}

In order to exclude that patient-related characteristics such as disease duration, age, gender, EDSS score, treatment duration or premedication had an effect on the DMF-attributed changes described above, epidemiological data of the patients were collected.

\subsubsection{Correlation of immune cell distribution and patient-related characteristics}

As shown in Figure 47, patient-related characteristics had no relevant influence on the frequency of most of the investigated immune cell subsets within the PBMC pool. However, one correlation was found between the frequency of $\mathrm{CD} 8^{+} \mathrm{T}$ cells and the age of the patients: The older the patients were, the lower the percentage of cytotoxic T cells was. As this effect was seen both in controls and DMF-treated patients and the mean patient age was almost similar in both patient groups (Table 1), it is very unlikely that patient age is the reason for the observed reduction of $\mathrm{CD}^{+} \mathrm{T}$ cells upon DMF.

\subsubsection{Correlation of $\mathbf{B}$ cell subsets, activation marker and molecules involved in antigen presentation and patient-related characteristics}

Regarding the different B cell subsets, representing the maturation process of those cells, there was no detectable correlation between any patient related data and the observed changes in B cell subset frequencies (Figure 48). The same was the case for the activation markers CD25, CD69 and CD 95 as shown in Figure 49 and the expression of CD40, CD80 and CD86 on B lymphocytes. Patient age, gender, EDSS score and premedication had no influence on these parameters. 
The only correlation found was that the longer control patients have had MS prior to the DMF therapy, the lower the CD40 expression was and the higher the CD86 expression was on B cells. Because of the complementary matching of the patient cohorts (Table 1), these observations did not influence the DMF-induced changes described above (Figure 50).

\subsubsection{Correlation of B cell cytokine production and patient-related characteristics}

By producing pro- and anti-inflammatory cytokines, B cells play a major role in the initiation and regulation of immune responses in the pathogenesis of MS. As shown above, DMF induces an anti-inflammatory shift of human B cells. Again, the only epidemiological parameter that correlates with the B cell-produced cytokines, is the disease duration. The longer control patients have had MS, the higher the TNF production and the lower the IL10 production was in B cells. As the patients in the two groups had almost similar disease durations (Table 1), these correlations do not influence the observed changes induced by DMF (Figure 51).

\subsubsection{Correlation of the cytokine production by $\mathrm{CD}^{+} 4^{+}$myeloid cells and patient- related characteristics}

In this analysis, there were only two relevant correlations between patient data and cytokine production of $\mathrm{CD}_{1} 4^{+}$myeloid cells. The older the patients were, the higher the IL-6 production of myeloid APC was in control patients. Also, the longer the disease duration of control patients was, the higher was the IL- 6 and the IL-10 production. Because of the proper patient matching, this observation should not affect the DMF-induced changes (Figure 52). 
A
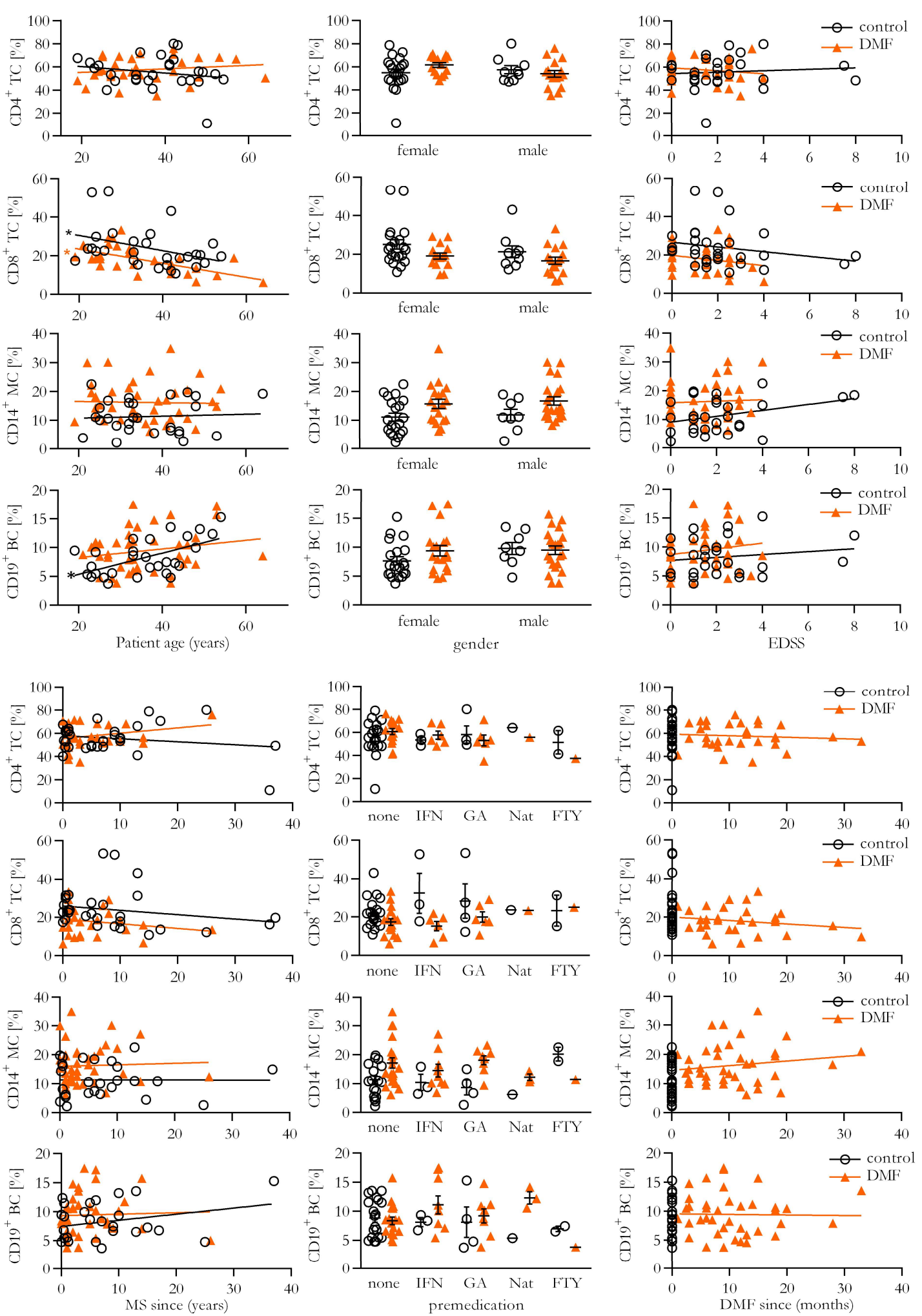

Figure 47: Correlation between cell frequencies and patient-related data. Cell frequencies of CD4 ${ }^{+} \mathrm{T}$ cells $(\mathrm{TC}), \mathrm{CD} 8^{+} \mathrm{TC}$, myeloid-derived phagocytes $\left(\mathrm{MC} ; \mathrm{CD}_{14}^{+}\right)$and $\mathrm{B}$ cells $\left(\mathrm{BC} ; \mathrm{CD} 19^{+}\right)$in human peripheral blood mononuclear cells (horizontally arranged) were correlated with patient age, gender and expanded disability status scale (EDSS) score (A) as well as disease duration, premedication (interferon (IFN), glatiramer acetate (GA), Natalizumab (Nat), fingolimod (FTY)) and treatment duration (B) using linear regression. Control multiple sclerosis (MS) patients (circles) were compared to dimethyl fumarate (DMF)-treated matches (triangles). Bars indicate mean \pm standard error of the mean. $(n=31$ (DMF); $n$ $=30($ control $)$; linear regression; $*=\mathrm{p}<0.05)$ 


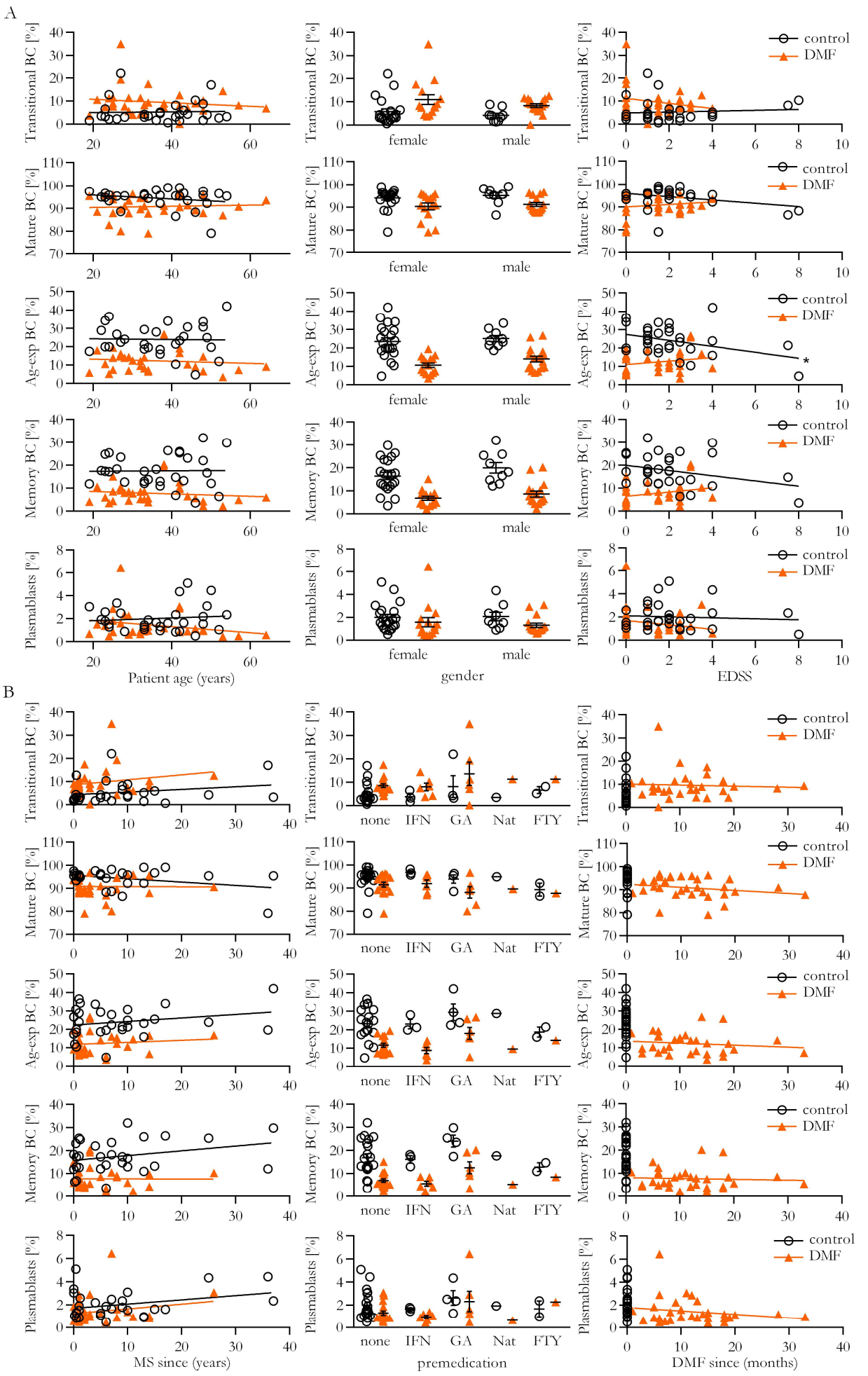

Figure 48: Correlation between B cell subsets and patient-related data. Frequencies of transitional B cells (BC) (CD24high $\left.\mathrm{CD} 38^{\text {high }}\right)$, mature $\mathrm{BC}\left(\mathrm{CD} 24^{\text {var }} \mathrm{CD} 38^{\text {low }}\right)$, antigen-experienced BC (Ag-exp; CD27+), memory BC (CD27var $\left.\mathrm{CD} 38^{-}\right)$and plasmablasts (CD20- CD27high $\mathrm{CD} 38^{\text {high }}$ ) in human peripheral blood mononuclear cells (horizontally arranged) were correlated with patient age, gender and expanded disability status scale (EDSS) score (A) as well as disease duration, premedication (interferon (IFN), glatiramer acetate (GA), Natalizumab (Nat), fingolimod (FTY)) and treatment duration (B) using linear regression. Control multiple sclerosis (MS) patients (circles) were compared to dimethyl fumarate (DMF)-treated matches (triangles). Bars indicate mean \pm standard error of the mean. $(n=31$ (DMF); $n=30$ (control); linear regression; $*=\mathrm{p}<0.05)$ 
A
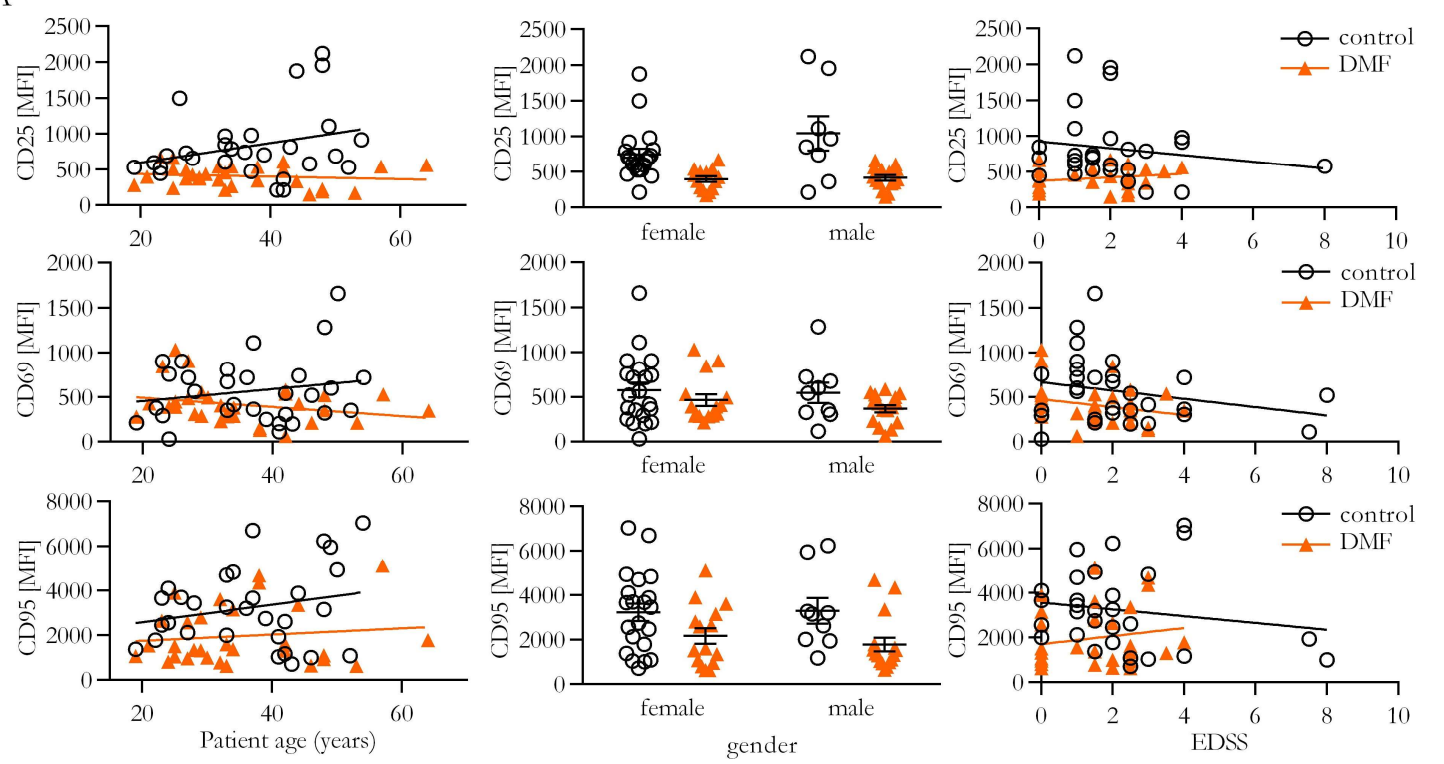

B
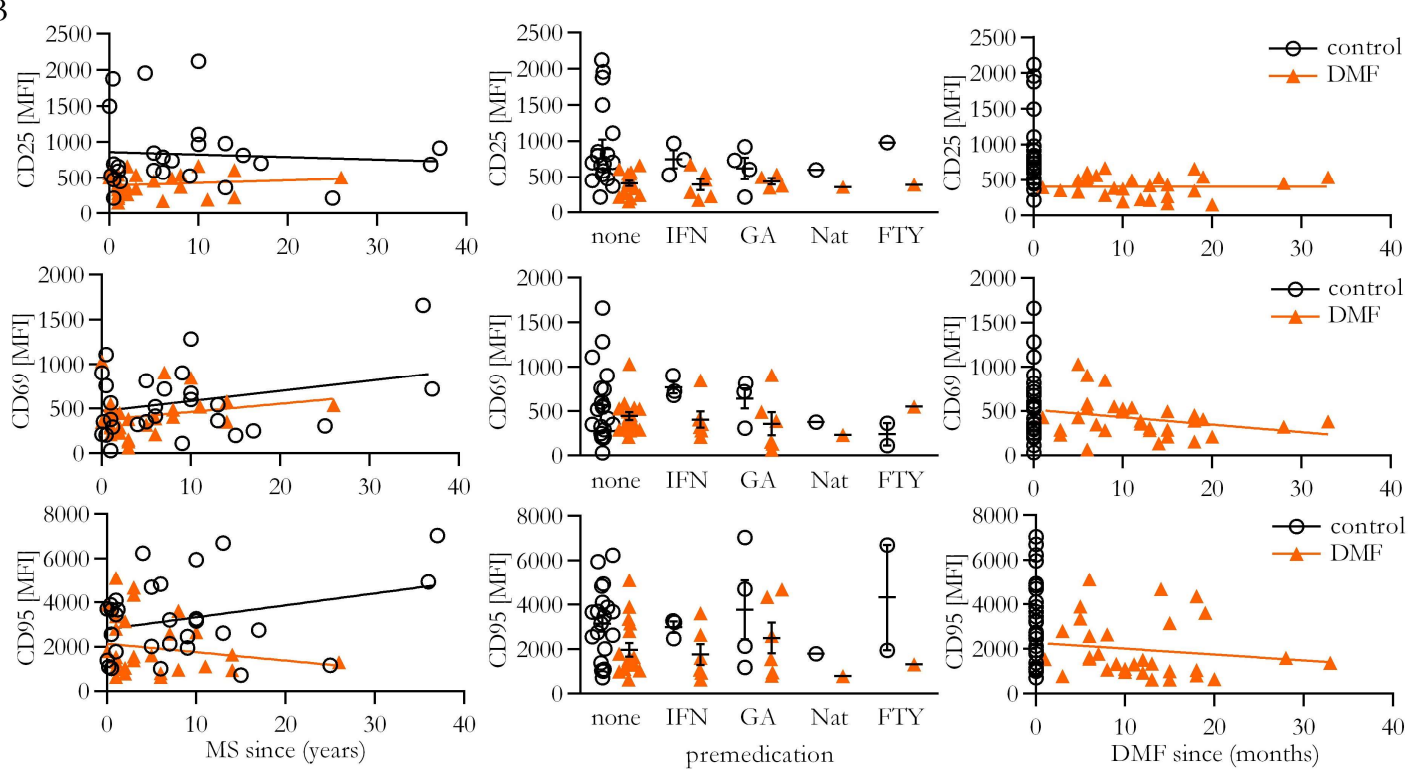

Figure 49: Correlation between cell $\mathrm{B}$ cells subsets and patient-related data. The expression of the activation markers CD25, CD69 and CD95 on human B cells (BC; CD19+) (horizontally arranged) were correlated with patient age, gender and expanded disability status scale (EDSS) score (A) as well as disease duration, premedication (interferon (IFN), glatiramer acetate (GA), Natalizumab (Nat), fingolimod (FTY)) and treatment duration (B) using linear regression. Control multiple sclerosis (MS) patients (circles) were compared to dimethyl fumarate (DMF)-treated matches (triangles). Bars indicate mean \pm standard error of the mean. $(n=31$ (DMF); $n=30$ (control); linear regression; not significant) 
A
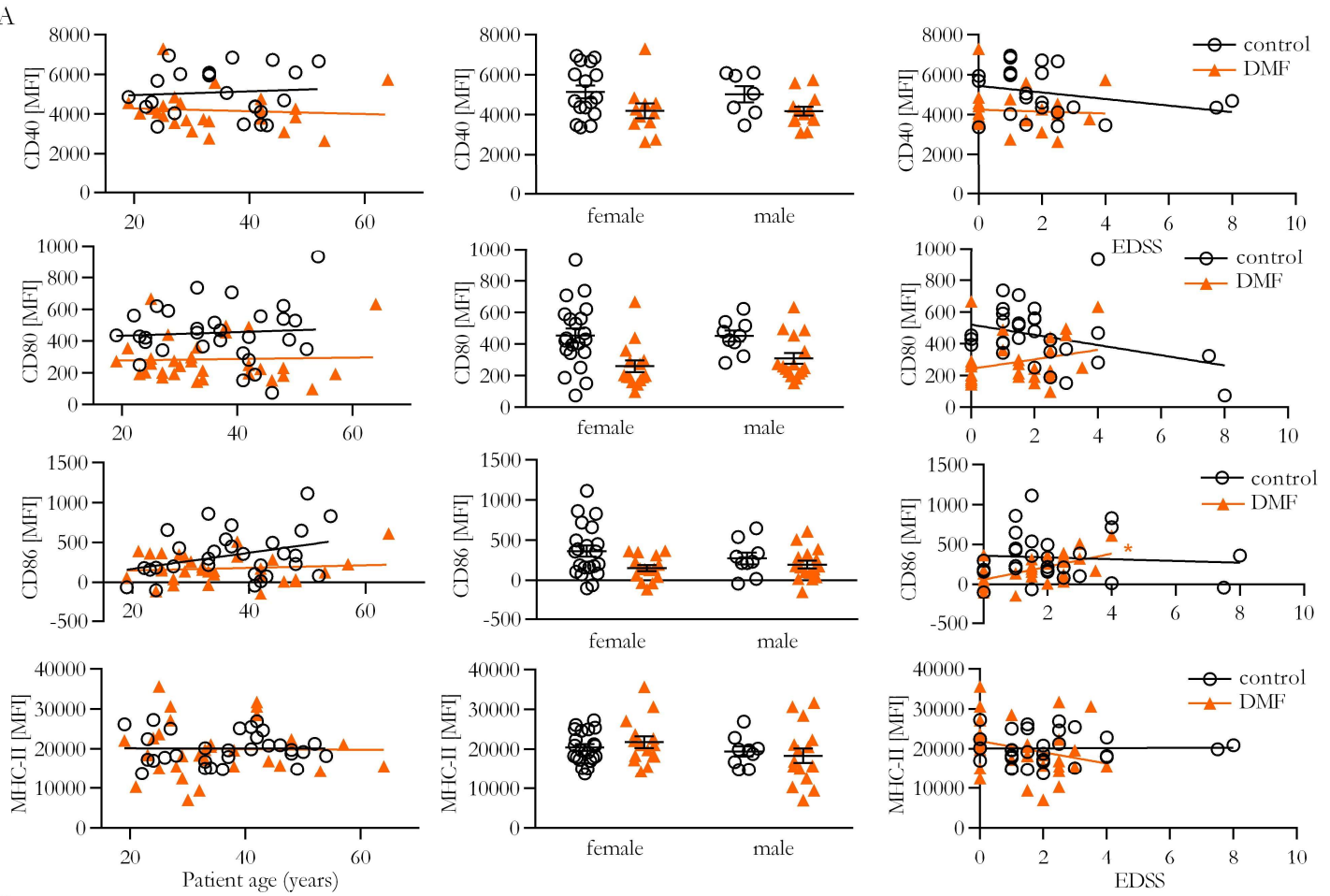

B
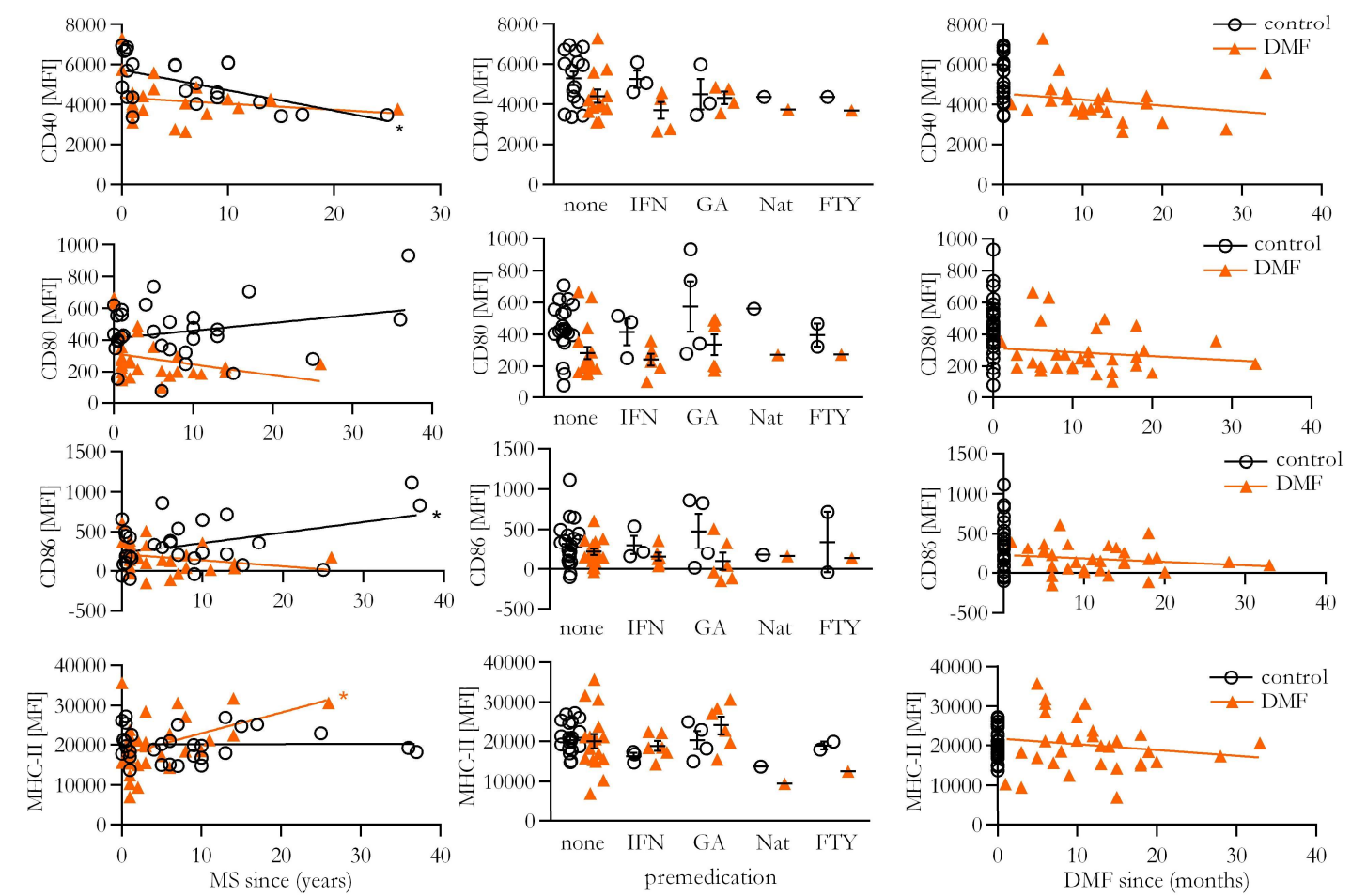

Figure 50: Correlation between antigen-presenting function relevant markers and patient-related data. The expression of the markers relevant for antigen presentation CD40, CD80, CD86 and major histocompatibility complex class II (MHCII) on human B cells (BC; CD19+) (horizontally arranged) were correlated with patient age, gender and expanded disability status scale (EDSS) score (A) as well as disease duration, premedication (interferon (IFN), glatiramer acetate (GA), Natalizumab (Nat), fingolimod (FTY)) and treatment duration (B) using linear regression. Control multiple sclerosis (MS) patients (circles) were compared to dimethyl fumarate (DMF)-treated matches (triangles). Bars indicate mean \pm standard error of the mean. ( $n=31$ (DMF); $n=30$ (control); linear regression; $*=\mathrm{p}<0.05)$ 
A
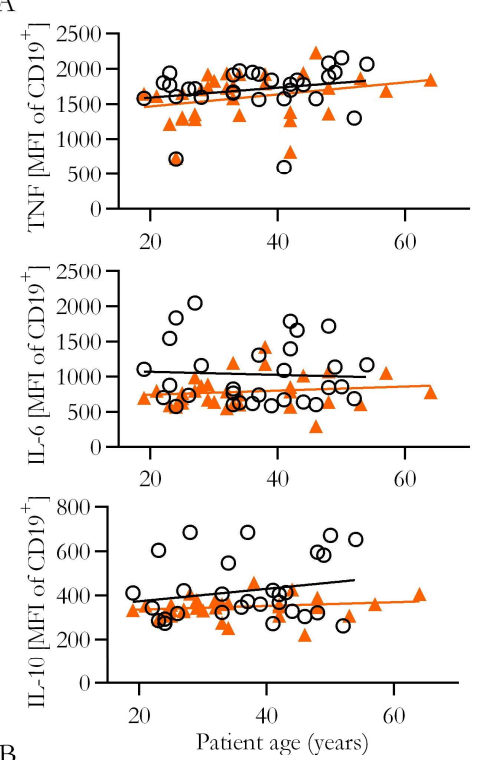

B
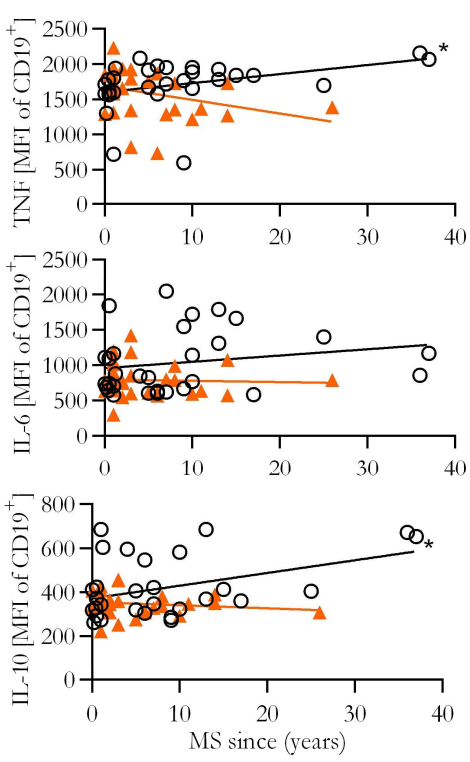
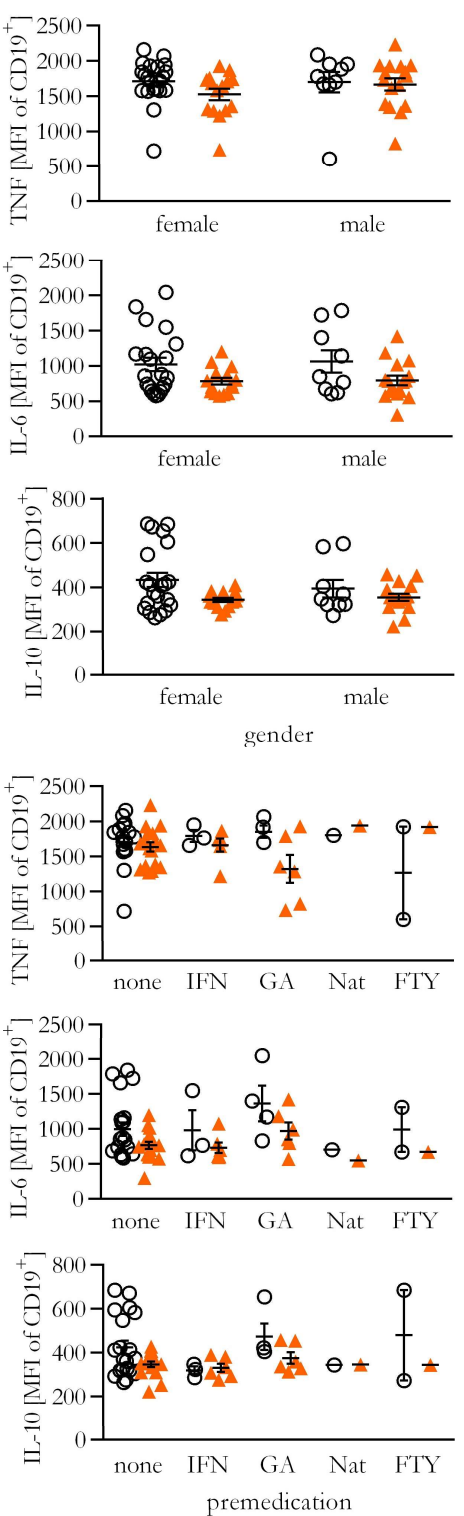
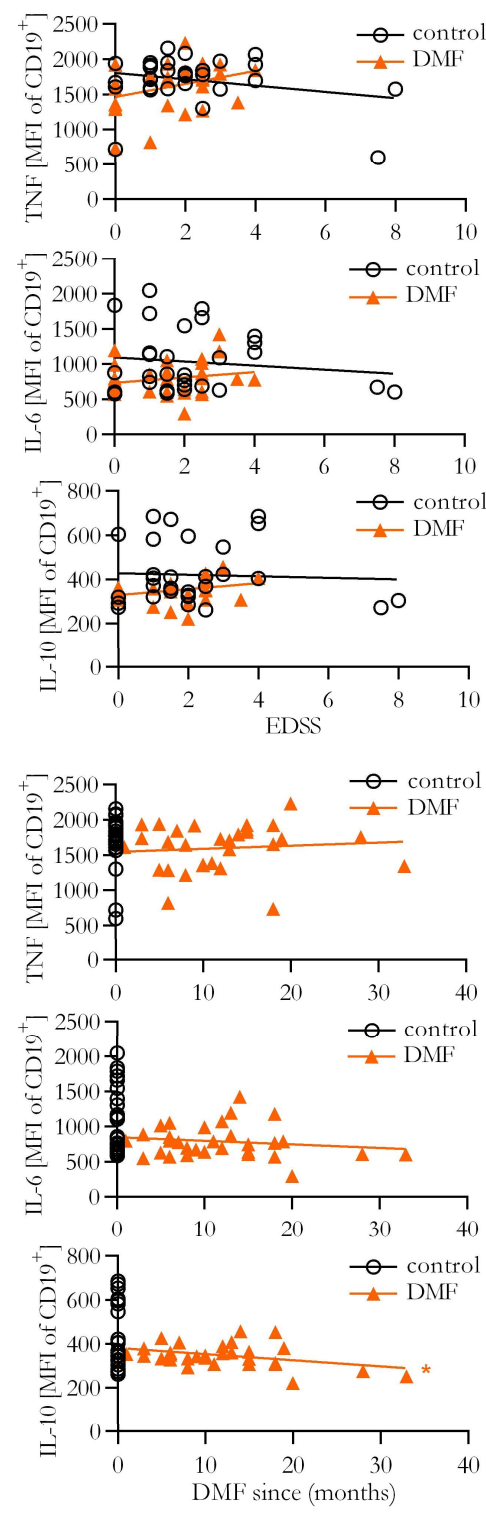

Figure 51: Correlation between B cell cytokine production and patient-related data. The production of the cytokines interleukin-6 (IL-6), interlekin-10 (IL-10) and tumor necrosis factor alpha (TNF) of human B cells (CD19+) (horizontally arranged) was correlated with patient age, gender and expanded disability status scale (EDSS) score (A) as well as disease duration, premedication (interferon (IFN), glatiramer acetate (GA), Natalizumab (Nat), fingolimod (FTY)) and treatment duration (B) using linear regression. Control multiple sclerosis (MS) patients (circles) were compared to dimethyl fumarate $(\mathrm{DMF})$-treated matches (triangles). Bars indicate mean \pm standard error of the mean. $(n=31(\mathrm{DMF}) ; n=30(\mathrm{control})$; linear regression; $*=\mathrm{p}<0.05)$ 
A
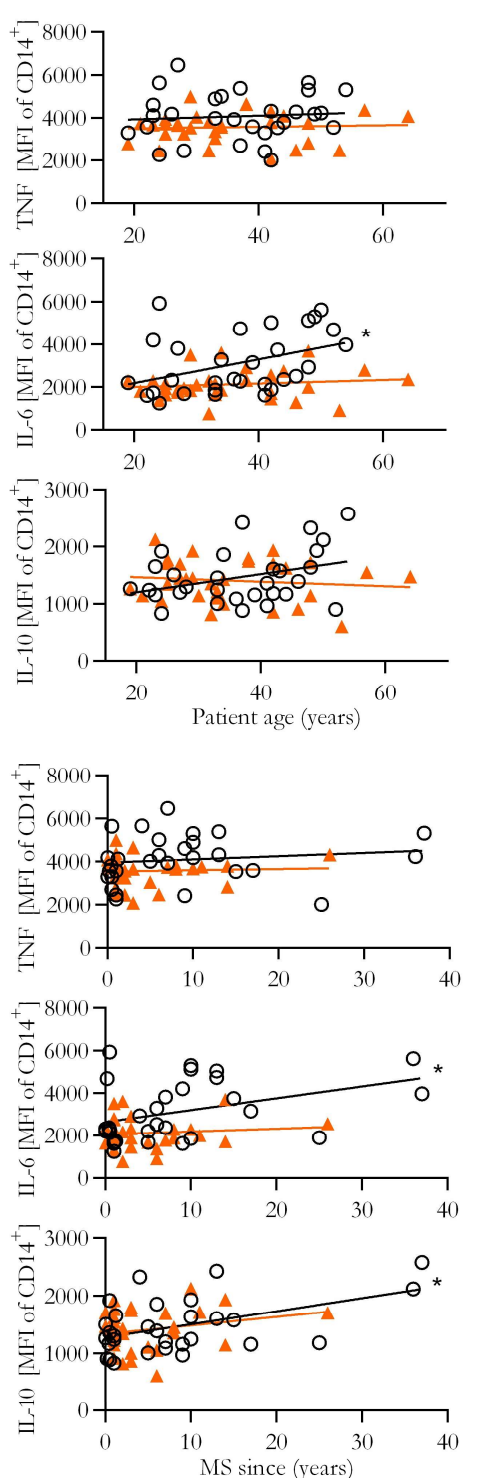
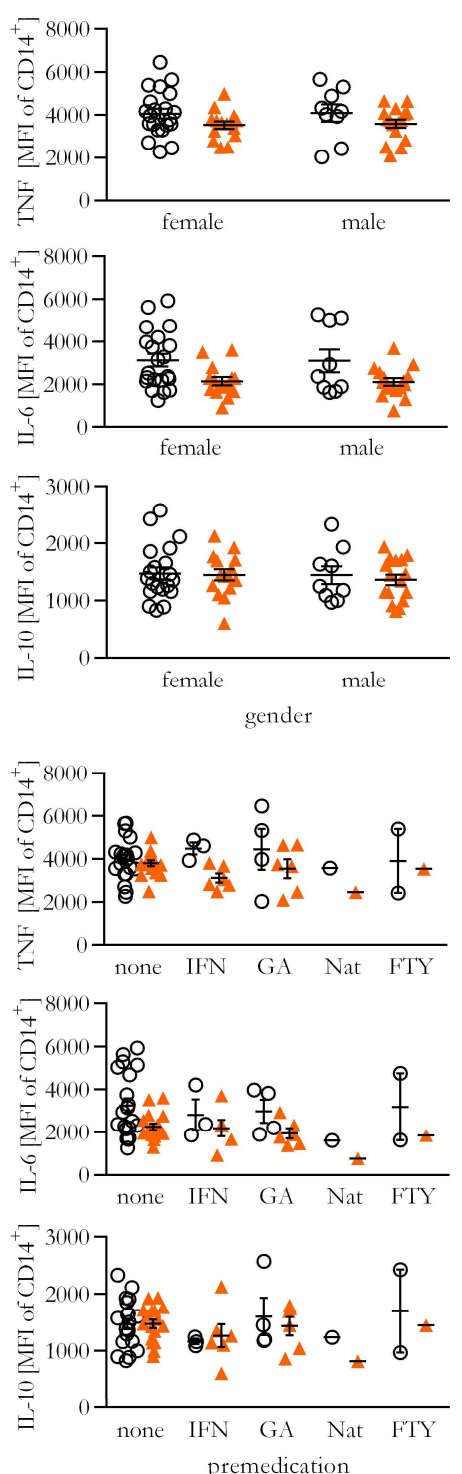
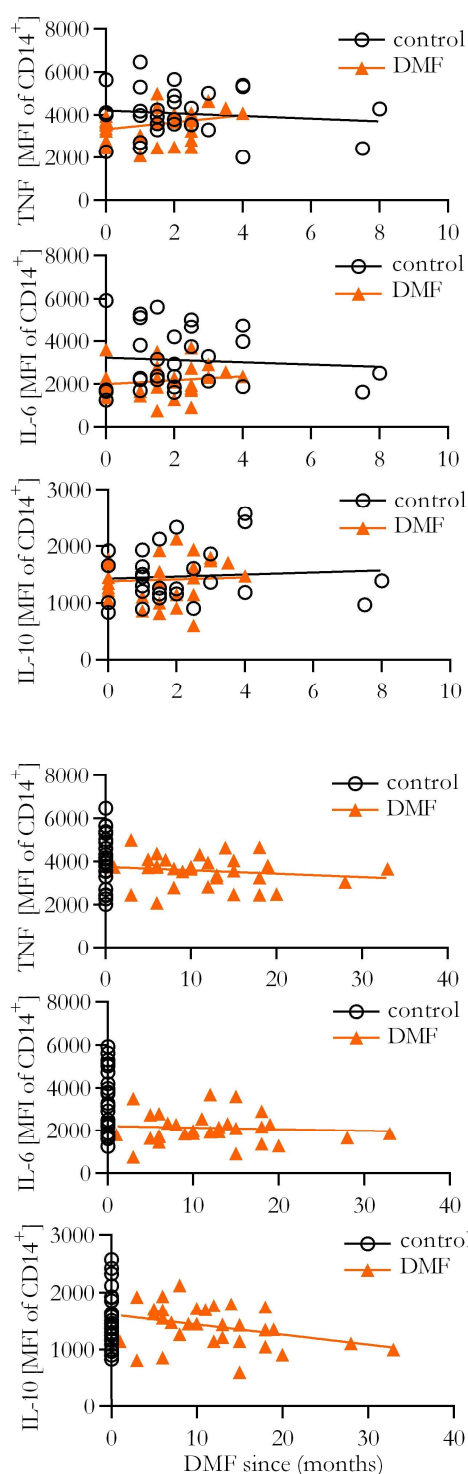

Figure 52: Correlation between myeloid-derived phagocyte cytokine production and patient-related data. The production of the cytokines interleukin-6 (IL-6), interlekin-10 (IL-10) and tumor necrosis factor alpha (TNF) of human CD14+ myeloid cells (horizontally arranged) was correlated with patient age, gender and expanded disability status scale (EDSS) score (A) as well as disease duration, premedication (interferon (IFN), glatiramer acetate (GA), Natalizumab (Nat), fingolimod (FTY)) and treatment duration (B) using linear regression. Control multiple sclerosis (MS) patients (circles) were compared to dimethyl fumarate (DMF)-treated matches (triangles). Bars indicate mean \pm standard error of the mean. $(n=31$ (DMF); $n=30$ (control); linear regression; $*=\mathrm{p}<0.05)$ 


\section{Discussion}

The study at hand was conducted to gain a deeper understanding of how DMF treatment affects the peripheral immune system of MS patients, with a major focus on B cells. There is strong evidence that $\mathrm{B}$ cells contribute to disease progression and to the occurrence of relapses in RRMS patients. Not least the success of B cell-depleting therapies proves their crucial role in the pathophysiology of MS (Hauser et al. 2017) and shows the necessity to investigate in greater detail whether already approved medications, such as DMF may influence this immune cell subset. With over 100,000 patients treated worldwide, DMF has become one of the most-prescribed medications in RRMS. However, its exact mode of action and its differential effects on B cells are still poorly understood. Thus, the current study aimed to dissect how DMF influences the phenotype and function of B lymphocytes. Therefore, blood samples of 31 DMF-treated subjects and 30 untreated controls were collected and flow cytometry and ELISA studies were performed to determine B cell maturation, activation, and differentiation. In summary, this study shows that DMF treatment affects in particular mature, differentiated B cell phenotypes, leading to their reduction within the total $\mathrm{B}$ cell pool. Further, it induces a diminished expression of activation markers and molecules involved in antigen presentation on the surface of $\mathrm{B}$ lymphocytes and reduces the production of pro-inflammatory cytokines. These findings suggest a strong DMF-mediated inhibitory effect on the B cell compartment while the quantitative frequency of $\mathrm{B}$ cells within the PBMC remains unaltered.

\subsection{The effect of dimethyl fumarate on B cells}

In the last decade, B cells gained decisive relevance in the pathogenesis of MS. Compared to healthy controls, RRMS patients have a higher frequency of pro-inflammatory B cells in their blood (Lundy et al. 2016) and B cell-depleting therapies showed promising results for both RRMS and PPMS (Juanatey et al. 2018). This raises the question whether DMF's therapeutic efficacy may also be in part mediated by an ablation of B lymphocytes. In line with the findings of Medina et al. (2017), the frequency of B lymphocytes was not altered upon DMF in the current examination, indicating that their loss is proportionate to the general decline of leukocytes upon DMF treatment and that beneficial effects of DMF treatment are not mediated by an exclusive, over-proportionate loss of B cells.

\subsubsection{B cell subsets}

As discussed above, the data at hand indicate that B cells were decreased proportionally to the general lymphocyte loss. However, it remains uncertain whether the composition of those remaining B cells may be altered by DMF treatment similar to the observations in $\mathrm{CD}^{+} \mathrm{T}_{\mathrm{h}}$ cells. To answer this question, $\mathrm{B}$ cell subsets were characterized according to their surface marker expression pattern and analyzed by flow cytometry: Transitional immature B 
cells (CD24 $\left.4^{\text {high }} \mathrm{CD} 27^{-} \mathrm{CD} 38^{\text {high }}\right)$, mature naive $\mathrm{B}$ cells (CD24 $4^{\text {high/int }} \mathrm{CD} 38^{\text {low/int }}$ ), antigen-experienced B cells $\left(\mathrm{CD} 27^{+}\right)$, memory B cells $\left(\mathrm{CD} 27^{\text {var }} \mathrm{CD} 38^{-}\right)$and plasmablasts (CD20- CD27 $\left.{ }^{\text {high }} \mathrm{CD} 38^{\text {high }}\right)$ were distinguished.

B cell development begins in the bone marrow, where B cells arise from common lymphoid progenitor cells and undergo various steps of maturation. Immature, so-called transitional B cells migrate through the blood to secondary lymphoid organs, such as spleen and lymph nodes, where they continue their differentiation. Thus, they represent the earliest maturation stage that can be found outside the bone marrow. The current study showed a significant increase in the frequency of these transitional B cells upon DMF treatment. Similar investigations performed by other authors showed that the absolute number of transitional B cells is not changed by DMF treatment (Smith et al. 2017; Li et al. 2017; Holm Hansen et al. 2018). However, these publications do not necessarily conflict with the data at hand: As the total B cell count decreases, an unchanged transitional B cell count is reflected by a higher percentage of those cells. A relative increase in transitional B cells has also been reported for IFN- $\beta$ and Fingolimod treatment (Longbrake and Cross 2016; Grützke et al. 2015), pointing towards a similar B cell attenuating effect of these drugs. Furthermore, the current study found that the higher frequency of transitional B cells is stable in long-term treatment, being in accordance with other publications (Lundy et al. 2016; Montes Diaz et al. 2018). It was shown that transitional $\mathrm{B}$ cells are able to exhibit anti-inflammatory functions through the production of the anti-inflammatory cytokine IL-10 (Blair et al. 2010). This suggests that the observed relative increase of transitional B cells may contribute to the observed general anti-inflammatory shift in cytokine production.

In secondary lymphoid organs, transitional B cells develop into mature B cells. The results at hand show a significant decline of this mature population upon the influence of DMF and indicate that the loss of mature B cells is over-proportionate in comparison to the decline of transitional B cells. This observation may point towards mature B cells being a major target of DMF and is in line with the observation by Li et al. (2017), who showed that the transitional/mature B cell ratio increases significantly upon DMF treatment. Further, in a set of in vitro experiments it was shown that the apoptosis of mature B cells, but not transitional $\mathrm{B}$ cells, is increased in the presence of DMF ( $\mathrm{Li}$ et al. 2017) indicating that they are potentially more vulnerable to DMF-induced ablation.

$B$ cells encounter antigens specifically via their B cell receptor and present them via MHC-II on their cell surface. When $\mathrm{CD}^{+}{ }^{+} \mathrm{T}$ cells recognize these antigens and interact with the $\mathrm{B}$ cell, both cell types start to proliferate and differentiate. Mediated by the cell-cell interaction, $B$ cells express CD27 on their surface. Hence, this molecule can be used to identify antigenexperienced cells. As shown in 3.3.2, the percentage of antigen-experienced B cells was strongly reduced in patients treated with DMF, being in agreement with other studies (Li et al. 2017; Lundy et al. 2016; Smith et al. 2017). In fact, many approved immunomodulatory drugs induce a decline of $\mathrm{CD} 27^{+} \mathrm{B}$ cells, which goes along with their clinical success (Baker 
et al. 2017). Therefore, this molecule may be a promising biomarker for treatment efficacy and indicates that DMF modulates the B cell pool in a favorable manner.

Antigen-experienced B cells that interact with T cells either undergo clonal expansion in the germinal center, become long-lived plasma cells or memory B cells. The latter can be germinal center-dependent or -independent. After a second exposure to their specific antigen, memory B cells generate an earlier and more effective humoral immune response than newly-activated B cells (Kurosaki et al. 2015). Thus, memory B cells are assumed to be highly relevant in the pathophysiology of MS and have been reported to be elevated in MS patients (Bar-Or et al. 2010). In this project, a reduced memory B cell frequency upon DMF treatment was observed, supporting the data of other authors (Li et al. 2017; Smith et al. 2017; Medina et al. 2017). A relative decrease in this subset has also been reported for glatiramer acetate, fingolimod and alemtuzumab treatment with an association between the reduction of memory B cells and the success of treatment (Longbrake and Cross 2016). This may be due to the fact that memory $B$ cells show a higher expression of costimulatory molecules and pro-inflammatory cytokines such as granulocyte macrophage colonystimulating factor and TNF (Bar-Or et al. 2001; Duddy et al. 2007), rendering them more effective APC for the generation of pathogenic T cells. This preferential loss of memory $\mathrm{B}$ cells may again be mediated by the preferential targeting of cells with a high metabolism (Kornberg et al. 2018). In addition, the inhibitory effect of DMF on T cells may indirectly contribute to the reduced memory B cell activation, as B cells receive less $T$ cell help reducing their activation.

Plasmablasts are the direct precursors of plasma cells in the blood. In contrast to plasma cells, they are still capable of rapid division and antigen presentation. Remaining in this state for some days, they either die or differentiate into adult plasma cells. While plasma cells are mainly found in the secondary lymphoid organs and the bone marrow, plasmablasts are more abundant in the peripheral blood. The present analysis revealed that DMF treatment reduces their frequency significantly, suggesting that plasmablasts are over-proportionally affected by DMF treatment. This may be due to their high metabolism and DMF's impact on metabolically highly active cells (Kornberg et al. 2018) or again indirectly mediated by the inhibition of $\mathrm{T}$ cells by DMF and the accompanying reduced interaction of $\mathrm{B}$ and $\mathrm{T}$ cells. However, these data are potentially in conflict to other studies reporting an unchanged absolute plasmablast count (Smith et al. 2017; Longbrake and Cross 2016). As the current investigation analyzed B cell frequency, direct comparison to this study is not possible.

Conclusively, the fact that DMF does not change B cell frequency but induces a shift towards less mature cells may be explained by the DMF-dependent modification of reactive cysteine residues. They are assumed to play a role in $\mathrm{B}$ cell maturation and have been reported to be one reason for the selective maturation-dependent targeting of certain B cell subsets (Nam and Lim 2016; Blewett et al. 2016). Also, it is a cysteine site of the GAPDH enzyme that becomes succinated and inactivated by DMF (Kornberg et al. 2018). It must be noted that 
future studies could have a more detailed look on the expression levels of activation markers and cytokine production in the distinct B cell subsets itself and examine whether they could be targeted more specifically.

\subsubsection{B cell survival}

To determine, if this effect on B cell subset frequencies is at least partly caused by the induction of cell death by apoptosis or necrosis of affected cells, this study compared the survival of PBMC isolated from control or DMF-treated patients and analyzed their difference in survival capacity.

Excluding necrotic cells and cell fragments by size exclusion, the data at hand indicate a significantly higher percentage of size-excluded, possibly ruptured or necrotic cells in DMF-treated samples. This effect was magnified when a stimulant was added. As it seems, DMF increases the vulnerability of B cells to undergo necrotic cell death or general rupture upon external cell stressors in the laboratory process.

In contrast, apoptosis is a well-regulated, organized process and triggered by certain cascades. Using Zombie dye positivity as criteria for dead cells after the exclusion of ruptured and necrotic cells, the current study found an increased proportion of dead cells upon DMF treatment and stimulation. Specifically, B cells showed an increased fraction of Zombie positive cells upon DMF treatment, while the frequency of 'dead' phagocytes was not affected. This suggests that the above discussed increase in myeloid cell frequency correlated with an improved survival.

Using Annexin V as an apoptotic marker, it was recently shown that B cell apoptosis is increased in vitro when DMF is given directly to the cells. This effect was present especially in mature B cells (Li et al. 2017; Montes Diaz et al. 2018). Moreover, DMF was capable of inducing apoptosis in human $\mathrm{T}$ cells in vitro (Treumer et al. 2003). However, the fact that apoptosis of $\mathrm{B}$ cells and $\mathrm{T}_{\mathrm{h}}$ cells was detected at unphysiologically high MMF concentrations of $20-30 \mu \mathrm{g} / \mathrm{ml}$ supports the theory that apoptosis may not be the only reason for the decreases of cell counts (Holm Hansen et al. 2018). Others may include the inhibition of maturation and metabolically high active cells (Kornberg et al. 2018).

\subsubsection{Activation state of $B$ cells}

Furthermore, this study aimed to elucidate if, apart from B cell subset frequency changes, DMF is also capable of changing the activation state of B cells. Upon encountering antigen or interaction with $\mathrm{T}$ cells, $\mathrm{B}$ cells upregulate certain surface molecules referred to as activation markers. To investigate DMF-induced changes of these molecules, B cells were stained for CD25, CD69, CD95 and CD150.

This analysis revealed a strongly reduced expression of all investigated activation markers on B lymphocytes when patients were treated with DMF. Moreover, the observed inhibition 
was stable or even intensified over time indicating that the effects of DMF on B cell activation are long lasting and effective. These findings can either be explained by an inhibited activation of B cells by DMF or the loss of activated cells. The fact that apoptosis rates were elevated in differentiated cells in vitro supports the latter theory (Montes Diaz et al. 2018; Li et al. 2017). Further, the preferential targeting of cells with a high turnover may explain the reduced frequency of B lymphocytes expressing activation markers (Kornberg et al. 2018). Alternatively, DMF may induce an arrest of B cell maturation by binding NF- $x$ B, inhibiting its translocation into the nucleus and its binding to the deoxyribonucleic acid (Gillard et al. 2015; Litjens et al. 2006; Loewe et al. 2002). To further elucidate these complex interactions, further studies with a more detailed surface marker expression on the specific B cell subsets themselves will be needed. For mainly B cell-mediated diseases, such as neuromyelitis optica spectrum disorder, the observed alterations might have a beneficial clinical relevance.

\subsubsection{Antigen presentation by $\mathrm{B}$ cells}

Being potent APC, B cells can specifically bind and internalize antigens through their B cell receptor, process them and present the linearized determinants on MHC-II molecules. For an effective interaction with $\mathrm{CD}^{+}{ }^{+} \mathrm{T}$ cells, costimulatory molecules like CD40, CD80 and CD86 are furthermore needed. Since it was shown that the MHC-II-restricted APC function of B cells is required for the induction of experimental CNS autoimmunity (Molnarfi et al. 2013), it is important to understand possible effects of DMF on this process. Hence, B cells of untreated and DMF-treated MS patients were stained for CD40, CD80, CD86 and MHCII. Similar to the activation markers, CD40, CD80 and CD86 were strongly downregulated upon DMF treatment. These results confirm recent in vitro data, where similar effects for CD40 and CD 80 were seen on B cells after incubation with $10 \mu \mathrm{M}$ DMF (Montes Diaz et al. 2018).

MHC-II remained unchanged in the cross-sectional samples but was slightly upregulation in the longitudinal analysis, with a trend towards a higher MHC-II expression the longer DMF was taken. This is in contrast to a study in mice that claimed that DMF treatment induces a MHC-II downregulation on B cells (Schulze-Topphoff et al. 2016) but in line with a recent EAE study showing that MHC-II expression remained unchanged upon DMF (Traub et al. 2019). A more recent in vitro investigation claimed that DMF downregulates the expression of MHC-II on purified B cells (Montes Diaz et al. 2018), but those experiments were conducted with unphysiologically high DMF concentrations of $10 \mu \mathrm{M}$ DMF. Apart from this, it can be questioned whether in vitro effects of DMF are at all relevant for the in vivo situation, as ingested DMF is promptly converted into its active metabolite MMF and thus only shortly present in the blood of treated patients(Schmidt et al. 2007). However, possible clinical consequences of this slight upregulation of MHC-II on B cells are difficult to predict as the interplay of the individual components in the process of antigen presentation (CD40, CD80, CD86, MHC-II) may be more relevant than the upregulation of a single molecule. 
Thus, the observed strong downregulation of costimulatory molecules in combination with the slight MHC-II upregulation may anyway result in a reduced B/T cell interaction with extenuated $\mathrm{T}$ cell activation. In fact, it has been shown that $\mathrm{B}$ cells isolated from DMF-treated mice activate $\mathrm{CD}^{+} \mathrm{T}$ cell to a lower extend than untreated controls (Traub et al. 2019), indicating that DMF has an dampening effect on the APC function of B cells. However, future functional studies with B cells isolated from DMF-treated patients would be desirable to confirm the observations in mice and to broaden the performed phenotypical analysis in MS patients.

\subsubsection{Cytokine production by B cells}

To gain a deeper understanding of the functional consequences of the observed DMF-induced phenotypical changes, cytokine production of B cells was determined. Proinflammatory cytokines, such as TNF and IL-6 as well as anti-inflammatory cytokines such as IL-10 are key mediators of pathogenic processes in MS. In this analysis, PBMC were incubated with $\mathrm{CpG}$, ionomycin and PMA to trigger cytokine production. Intracellular cytokine staining and ELISA measurements were used to quantify the amount of produced and secreted interleukins.

Both longitudinal and cross-sectional patient samples revealed an inhibiting effect of DMF on pro-inflammatory TNF and IL-6 production, but presumably no effect on IL-10 being in line with other recent publications ( $\mathrm{Li}$ et al. 2017; Smith et al. 2017). As TNF and IL-6 promote inflammatory $\mathrm{T}$ cell responses ( $\mathrm{Li}$ et al. 2015), DMF's effect on B cells may indirectly also fortify the inhibition of T cell activation. Similar to DMF, TNF and IL-6 reduction and a slight elevation of IL-10 was also described for other effective drugs, such as glatiramer acetate, fingolimod and mitoxanthrone (Longbrake and Cross 2016).

\subsection{The effects of dimethyl fumarate on other leukocytes}

Not only B cells, but rather the peripheral immune system as a whole is assumed being essential for the development and progression of MS. Thus, current approved therapies aim to alter the immune response in a broader manner by depleting cells, affecting their migration, proliferation or function to slow down MS progression (Sedal et al. 2017; Vargas and Tyor 2017). Accordingly, several studies demonstrated that DMF treatment reduces the absolute lymphocyte count in peripheral blood of MS patients by approximately $30 \%$ in the first year of treatment (Fox et al. 2016). However, it has not been fully elucidated whether this cell reduction effects all leukocytes to the same extent or whether DMF treatment changes their relative composition by disproportionately affecting individual immune cell subsets. To answer this question, PBMC of DMF-treated and untreated controls were isolated and stained for immune cell lineage marker to identify $\mathrm{T}_{\mathrm{h}}$ cells $\left(\mathrm{CD} 4^{+}\right)$, cytotoxic $\mathrm{T}$ cells $\left(\mathrm{CD} 8^{+}\right)$, myeloid-derived phagocytes $\left(\mathrm{CD} 14^{+}\right)$and B lymphocytes $\left(\mathrm{CD} 19^{+}\right)$. 


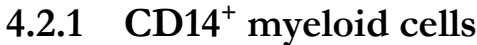

Next to B cells, professional APC such as myeloid cells are required for the activation of T cells. CD14 $4^{+}$myeloid-derived phagocytes, such as monocytes, macrophages and a subset of dendritic cells circulate in the peripheral blood or invade tissue to encounter and internalize antigens. They process these molecules to linearized determinants and present them via MHC-II to T cells. This function is important in the concept of the peripheral activation of pathogenic $\mathrm{CD}^{+} \mathrm{T}$ cells in MS pathophysiology. Hence, analysis of $\mathrm{CD}^{+} 4^{+}$cells was performed in the present investigation: Upon DMF treatment, there was a significant increase in the frequency of $\mathrm{CD} 14^{+}$cells within all PBMC, being in line with the results of other studies that found an absolute increase of $\mathrm{CD}_{14}{ }^{+}$cell counts upon DMF (Michell-Robinson et al. 2016; Montes Diaz et al. 2018).

Investigating the phenotype of these phagocytes in detail, DMF treatment had no detectable effect on their activation, as CD95 and CD150 were not altered, but led to a slight reduction in the expression of the co-stimulatory molecules CD40, CD80 and CD86 being in line with the in vitro data published by Zhu et al. (2002). In line with this, DMF decreases the maturation and antigen-presenting capacity of phagocytes through the suppression of NF$x \mathrm{~B}$ and ERK1/2 pathways in vitro (Mills et al. 2018). Although the MHC-II expression on $\mathrm{CD} 14^{+}$cells remained unchanged both in the cross-sectional and the longitudinal samples, the correlation with treatment duration suggested that, similar to B cells, MHC-II expression increases slowly but steadily. Regarding the cytokine profile of $\mathrm{CD}_{14}{ }^{+}$myeloid cells upon DMF treatment, a reduction of TNF and IL-6 production was observed, while the synthesis of IL-10 remained unaltered. Those results are in line with the in vitro findings of other studies showing that myeloid cell cytokine production undergoes an anti-inflammatory shift upon DMF exposure (Michell-Robinson et al. 2016; Lehmann et al. 2007). However, the functional consequences of these phenotypical findings need to be assessed in ensuing studies. One must take notice of the fact, that DMF generally dampens the activation, differentiation and pro-inflammatory cytokine production of $\mathrm{CD}^{+}{ }^{+} \mathrm{T}$ cells (Montes Diaz et al. 2018).

The inhibitory effect on activation, co-stimulation and cytokine production of $\mathrm{CD} 14^{+}$ myeloid APC observed in the present study matches with the assumption of others that DMF mediates a shift towards the presumed anti-inflammatory M2 phenotype of macrophages (Schulze-Topphoff et al. 2016). Indirectly, properties of B cells such as antigen presentation and cytokine production influence the response of myeloid cells both in a proinflammatory and a regulatory manner (Häusser-Kinzel and Weber 2019). These changes then may translate into a reduced activation of pathogenic $\mathrm{T}$ cells. Thus, one can assume that the anti-inflammatory effects on B cells and CD $14^{+}$myeloid cells, together with direct effects on T cells, converge and intensify the efficacy of DMF. Taken together, these finding suggest that DMF has an overall beneficial effect on the $\mathrm{CD}_{1} 4^{+}$cell compartment despite the quantitative increase (Schulze-Topphoff et al. 2016). 


\subsubsection{T cells}

Evidence suggests that $\mathrm{T}$ cells are major contributors to MS pathology, as they migrate to the CNS upon activation by peripheral and CNS-resident APC. There, they are assumed to contribute to the initiation of processes that trigger demyelinating and inflammatory cascades resulting in the development of focal lesions typical for MS. Early-active lesions are characterized by the abundance of $\mathrm{CD}^{+}$and $\mathrm{CD} 8^{+} \mathrm{T}$ cells. Hence, $\mathrm{T}$ cells are of major interest when evaluating the efficacy of new MS medications.

In the present study, no significant changes in the overall frequency of $\mathrm{CD}^{+} \mathrm{T}_{\mathrm{h}}$ cells could be observed upon DMF treatment, being in accordance with the findings of Spencer et al. (2015) and Fleischer et al. (2017). However, a recent study suggests that DMF reduces the frequency of memory and effector memory $\mathrm{CD}^{+} \mathrm{T}$ cells as well as their production of pro-inflammatory cytokine, suggesting an overall inhibiting effect of DMF on the $\mathrm{CD}^{+} \mathrm{T}$ cell compartment (Montes Diaz et al. 2018). As T cells require help of professional APC, these changes may be mediated by the abovementioned inhibition of $\mathrm{B}$ cells or the shift of myeloid cells towards the M2 phenotype. However, it is also plausible that DMF has direct effects on T cells. Wu et al. (2017) showed that in vitro treatment of T cells with DMF leads to a selective reduction of memory $\mathrm{T}$ cells and an increase of the $\mathrm{CD}^{+} / \mathrm{CD}^{+} \mathrm{T}$ cell ratio.

Next to $\mathrm{CD}^{+} \mathrm{T}_{\mathrm{h}}$ cells, $\mathrm{CD} 8^{+} \mathrm{T}$ cells are suggested to contribute to CNS damage. Cytotoxic $\mathrm{T}$ cells are assumed to contribute to axonal loss and demyelination by misleadingly directly attacking glial cells presenting autoantigens by MHC-I molecules (Liu et al. 2007). In doing so, they are thought to fortify and accelerate inflammatory and demyelinating processes in the development of MS. The results at hand show that the frequency of $\mathrm{CD}^{+} \mathrm{T}$ cells decreased upon DMF treatment in MS patients both in the longitudinal and the crosssectional analysis. Importantly, the $\mathrm{CD}^{+} \mathrm{T}$ cell frequency tended to keep declining the longer DMF was taken, suggesting a persistent and long-lasting effect. These observations are in line with former reports (Spencer et al. 2015; Fleischer et al. 2017). As $\mathrm{CD}^{+} \mathrm{T}$ cell frequencies are stable upon DMF treatment, but $\mathrm{CD}^{+} \mathrm{T}$ cell frequencies decline, one can assume that the reduction is disproportionate, resulting in an increased $\mathrm{CD} 4^{+} / \mathrm{CD} 8^{+} \mathrm{T}$ cell ratio.

Concluding, as cells were affected to a different extend, DMF may cause apoptosis through mechanisms or factors that are not equally present in all cells. Further investigation of DMF's exact mode of action may help explaining this phenomenon. Most recently, it was suggested that DMF interferes with the glycolysis of the immune cells by inhibiting the enzyme GAPDH (Kornberg et al. 2018). This may explain why the decrease of immune cells differs between the cell types; possibly due to different activation and metabolism levels. Moreover, Nrf2-dependent mechanisms are thought to contribute to DMF-mediated effects (Litjens et al. 2006). 


\subsection{Outlook}

The findings of this study reveal that DMF has broad inhibiting effects on B cell survival, maturation, activation and cytokine production, with mature, antigen-experienced and memory B cells being the major targets. Most prominently, the frequency of CD27 expressing cells is reduced by DMF. This molecule is mainly found on activated and memory B cells. Interestingly, it was shown, that the efficacy of other established MS medications correlates with a reduction of the $\mathrm{CD} 27^{+}$B cell subset (Baker et al. 2017). Hence, one can hypothesize that these cells are a favorably target in the immunotherapy of MS. In contrast to a general depletion of all B cells, a specific targeting of CD27 B cells would leave the beneficial, regulatory $\mathrm{B}$ cells unaffected. Therefore, a molecular antibody against $\mathrm{CD} 27^{+} \mathrm{B}$ cells seems favorable. However, the fact that CD27 is also expressed as a co-stimulatory molecule on $\mathrm{T}$ cells impedes its implementation. That's why to this date DMF, being relatively safe and well-tolerated, seems to be a good option for the relative reduction of pathogenic, activated B cells.

Recently approved B cell depleting therapies are very effective and suggest that B cells are major contributors to disease activity. As MS is a chronic disease that requires a long-lasting therapy, an effective B cell depleting therapy is associated with a high risk for serious side effects. Further, it is not fully understood by which phenotype B cells re-appear after therapy cessation and which factors trigger their pathogenic differentiation. Due to its extensive effects on this B cell subset, DMF might be a potential safe follow-up therapy to prevent (re-) activation of pro-inflammatory B cells.

Summing up, the oral agent DMF has been shown to exert various effects on the functional phenotype of B cells. As these days the mode of action is more and more being elucidated, its impact on distinct parts of the immune response needs to be clarified in a more detailed way. Also, long-term effects remain poorly understood. However, DMF, being a relatively safe oral drug, has the potential to play a major future role in the treatment of MS through sole application or follow-up treatment. 


\section{Summary}

Although the oral agent DMF was approved for the treatment of RRMS in 2014 and demonstrably reduces relapse rates and disability progression, its effect on B cells, which are now thought to be major contributors to disease activity, remains poorly understood. Therefore, PBMC of 31 DMF-treated and 30 treatment-naïve relapsing MS patients were analyzed both cross-sectionally and longitudinally using flow cytometry. Additional to intracellular cytokine staining, the supernatant was analyzed for cytokine concentrations using ELISA.

DMF treatment did not alter the B cell frequency within the PBMC pool. However, DMF reduced the survivability of cells, as it increased the number of size-excluded PBMC and dead B cells. Early transitional B cell frequency was increased, whereas mature, antigen-experienced and memory B cell frequencies diminished upon treatment. The same was the case for plasmablasts, indicating a shift towards less inflammatory cells. This shift was also seen in the evaluation of the expression levels of the activation markers CD25, CD69, CD95 and CD150 on B cells: This fits to the recent discovery that DMF affects mainly active immune cells with a high metabolic turnover. While DMF also dampened the costimulatory molecules CD40, CD80 and CD86, MHC-II surprisingly increased upon treatment. Functionally, DMF dampened the production of the pro-inflammatory cytokines IL-6 and TNF in B cells. Upon treatment, the observed effects were stable or even intensifying.

Future studies may investigate the phenotypical changes of the distinct B cell subsets, the functional consequences of the altered antigen presentation and long-term effects of DMF treatment. To sum up, this examination revealed that DMF has broad suppressive effects on pro-inflammatory B cell functions, possibly partly explaining the clinical success of DMF, possible drug combinations, treatment sequences or new indications of DMF in the treatment of MS and other autoimmune diseases. 


\section{References}

Akiba H, Nakano H, Nishinaka S, Shindo M, Kobata T, Atsuta M, Morimoto C, Ware CF, Malinin NL, Wallach D et al. (1998): CD27, a Member of the Tumor Necrosis Factor Receptor Superfamily, Activates NF- $\varkappa$ B and Stress-activated Protein Kinase/c-Jun Nterminal Kinase via TRAF2, TRAF5, and NF- $x$ B-inducing Kinase. J Biol Chem 273, 13353-13358

Albrecht P, Bouchachia I, Goebels N, Henke N, Hofstetter HH, Issberner A, Kovacs Z, Lewerenz J, Lisak D, Maher P et al. (2012): Effects of dimethyl fumarate on neuroprotection and immunomodulation. J Neuroinflammation $\underline{9}, 163$

Altmeyer PJ, Matthes U, Pawlak F, Hoffmann K, Frosch PJ, Ruppert P, Wassilew SW, Horn T, Kreysel HW, Lutz G (1994): Antipsoriatic effect of fumaric acid derivatives.

Results of a multicenter double-blind study in 100 patients. J Am Acad Dermatol $\underline{30}$, 977981

Baker D, Marta M, Pryce G, Giovannoni G, Schmierer K (2017): Memory B Cells are Major Targets for Effective Immunotherapy in Relapsing Multiple Sclerosis. EBioMedicine $\underline{16}, 41-50$

Banki K, Colombo E, Sia F, Halladay D, Mattson DH, Tatum AH, Massa PT, Phillips PE, Perl A (1994): Oligodendrocyte-specific expression and autoantigenicity of transaldolase in multiple sclerosis. J Exp Med 180, 1649-1663

Barnett MH, Prineas JW (2004): Relapsing and remitting multiple sclerosis: pathology of the newly forming lesion. Ann Neurol $\underline{55}, 458-468$

Bar-Or A, Oliveira EM, Anderson DE, Krieger JI, Duddy M, O'Connor KC, Hafler DA (2001): Immunological memory: contribution of memory B cells expressing costimulatory molecules in the resting state. J Immunol 167, 5669-5677

Bar-Or A, Fawaz L, Fan B, Darlington PJ, Rieger A, Ghorayeb C, Calabresi PA, Waubant E, Hauser SL, Zhang J et al. (2010): Abnormal B-cell cytokine responses a trigger of T-cellmediated disease in MS? Ann Neurol 67, 452-461

Bar-Or A, Pachner A, Menguy-Vacheron F, Kaplan J, Wiendl H (2014): Teriflunomide and its mechanism of action in multiple sclerosis. Drugs $\underline{74}$, 659-674

Barr TA, Shen P, Brown S, Lampropoulou V, Roch T, Lawrie S, Fan B, O'Connor RA, Anderton SM, Bar-Or A et al. (2012): B cell depletion therapy ameliorates autoimmune disease through ablation of IL-6-producing B cells. J Exp Med 209, 1001-1010

Bartholomäus I, Kawakami N, Odoardi F, Schläger C, Miljkovic D, Ellwart JW, Klinkert WEF, Flügel-Koch C, Issekutz TB, Wekerle H et al. (2009): Effector T cell interactions with meningeal vascular structures in nascent autoimmune CNS lesions. Nature $\underline{462}, 94-98$ 
Bhatia S, Edidin M, Almo SC, Nathenson SG (2006): B7-1 and B7-2: similar costimulatory ligands with different biochemical, oligomeric and signaling properties. Immunol Lett $\underline{104}$, $70-75$

Blair PA, Noreña LY, Flores-Borja F, Rawlings DJ, Isenberg DA, Ehrenstein MR, Mauri C (2010): CD19(+)CD24(hi)CD38(hi) B cells exhibit regulatory capacity in healthy individuals but are functionally impaired in systemic Lupus Erythematosus patients. Immunity $\underline{32}$, 129-140

Blewett MM, Xie J, Zaro BW, Backus KM, Altman A, Teijaro JR, Cravatt BF (2016): Chemical proteomic map of dimethyl fumarate-sensitive cysteines in primary human $\mathrm{T}$ cells. Sci Signal $\underline{9}$, rs10

Bruck W, Stadelmann C (2003): Inflammation and degeneration in multiple sclerosis. Neurol Sci 24 Suppl 5, S265-S267

Burton JM, O'Connor PW, Hohol M, Beyene J (2012): Oral versus intravenous steroids for treatment of relapses in multiple sclerosis. Cochrane Database Syst Rev $\underline{12}$, CD006921

Cambiaggi C, Scupoli MT, Cestari T, Gerosa F, Carra G, Tridente G, Accolla RS (1992): Constitutive expression of CD69 in interspecies T-cell hybrids and locus assignment to human chromosome 12. Immunogenetics $\underline{36}, 117-120$

Chen C, Gault A, Shen L, Nabavi N (1994): Molecular cloning and expression of early T cell costimulatory molecule-1 and its characterization as B7-2 molecule. J Immunol $\underline{152}$, 4929-4936

Chen H, Assmann JC, Krenz A, Rahman M, Grimm M, Karsten CM, Kohl J, Offermanns S, Wettschureck N, Schwaninger M (2014): Hydroxycarboxylic acid receptor 2 mediates dimethyl fumarate's protective effect in EAE. J Clin Invest 124, 2188-2192

Compston A, Coles A (2002): Multiple sclerosis. Lancet $\underline{\text { 359, }}$, 1221-1231

Compston A, Coles A (2008): Multiple sclerosis. Lancet $\underline{372,1502-1517}$

Connick P, Angelis F de, Parker RA, Plantone D, Doshi A, John N, Stutters J, MacManus D, Prados Carrasco F, Barkhof F et al. (2018): Multiple Sclerosis-Secondary Progressive Multi-Arm Randomisation Trial (MS-SMART): a multiarm phase IIb randomised, doubleblind, placebo-controlled clinical trial comparing the efficacy of three neuroprotective drugs in secondary progressive multiple sclerosis. BMJ Open $\underline{8}$, e021944

Cross AH, Naismith RT (2014): Established and novel disease-modifying treatments in multiple sclerosis. J Intern Med 275, 350-363

Cross SA, Cook DR, Chi AWS, Vance PJ, Kolson LL, Wong BJ, Jordan-Sciutto KL, Kolson DL (2011): Dimethyl fumarate, an immune modulator and inducer of the antioxidant response, suppresses HIV replication and macrophage-mediated neurotoxicity: A novel candidate for HIV neuroprotection. J Immunol 187, 5015-5025 
Dorstyn DS, Roberts RM, Murphy G, Haub R (2017): Employment and multiple sclerosis: A meta-analytic review of psychological correlates. J Health Psychol 24, 38-51

Duddy M, Niino M, Adatia F, Hebert S, Freedman M, Atkins H, Kim HJ, Bar-Or A (2007): Distinct effector cytokine profiles of memory and naive human B cell subsets and implication in multiple sclerosis. J Immunol 178, 6092-6099

Faissner S, Nikolayczik J, Chan A, Hellwig K, Gold R, Yoon M-S, Haghikia A (2016): Plasmapheresis and immunoadsorption in patients with steroid refractory multiple sclerosis relapses. J Neurol 263, 1092-1098

Fernández O, Calleja-Hernández MA, Meca-Lallana J, Oreja-Guevara C, Polanco A, PérezAlcántara F (2017): Estimate of the cost of multiple sclerosis in Spain by literature review. Expert Rev Pharmacoecon Outcomes Res 17, 321-333

Filippi M, Rocca MA, Ciccarelli O, Stefano N de, Evangelou N, Kappos L, Rovira A, Sastre-Garriga J, Tintorè M, Frederiksen JL et al. (2016): MRI criteria for the diagnosis of multiple sclerosis: MAGNIMS consensus guidelines. Lancet Neurol 15, 292-303

Fillatreau S, Sweenie CH, McGeachy MJ, Gray D, Anderton SM (2002): B cells regulate autoimmunity by provision of IL-10. Nat Immunol $\underline{3}, 944-950$

Fischer MT, Sharma R, Lim JL, Haider L, Frischer JM, Drexhage J, Mahad D, Bradl M, van Horssen J, Lassmann H (2012): NADPH oxidase expression in active multiple sclerosis lesions in relation to oxidative tissue damage and mitochondrial injury. Brain $\underline{135}$, 886-899 Fleischer V, Friedrich M, Rezk A, Bühler U, Witsch E, Uphaus T, Bittner S, Groppa S, Tackenberg B, Bar-Or A et al. (2017): Treatment response to dimethyl fumarate is characterized by disproportionate CD8+ T cell reduction in MS. Mult scler 24, 632-641 Fox RJ, Miller DH, Phillips JT, Hutchinson M, Havrdova E, Kita M, Yang M, Raghupathi K, Novas M, Sweetser MT et al. (2012): Placebo-controlled phase 3 study of oral BG-12 or glatiramer in multiple sclerosis. N Engl J Med $\underline{367}, 1087-1097$

Fox RJ, Chan A, Gold R, Phillips JT, Selmaj K, Chang I, Novas M, Rana J, Marantz JL, Ambler Z et al. (2016): Characterizing absolute lymphocyte count profiles in dimethyl fumarate-treated patients with MS: Patient management considerations. Neurol Clin Pract $\underline{6}, 220-229$

Franciotta D, Salvetti M, Lolli F, Serafini B, Aloisi F (2008): B cells and multiple sclerosis. Lancet Neurol ㅁ, 852-858

Fraussen J, Claes N, Bock L de, Somers V (2014): Targets of the humoral autoimmune response in multiple sclerosis. Autoimmun Rev $\underline{13}, 1126-1137$

Ghoreschi K, Brück J, Kellerer C, Deng C, Peng H, Rothfuss O, Hussain RZ, Gocke AR, Respa A, Glocova I et al. (2011): Fumarates improve psoriasis and multiple sclerosis by inducing type II dendritic cells. J Exp Med 208, 2291-2303 
Gillard GO, Collette B, Anderson J, Chao J, Scannevin RH, Huss DJ, Fontenot JD (2015): DMF, but not other fumarates, inhibits NF- $x \mathrm{~B}$ activity in vitro in an Nrf2-independent manner. J Neuroimmunol 283, 74-85

Gold R, Kappos L, Arnold DL, Bar-Or A, Giovannoni G, Selmaj K, Tornatore C, Sweetser MT, Yang M, Sheikh SI et al. (2012): Placebo-controlled phase 3 study of oral BG-12 for relapsing multiple sclerosis. N Engl J Med $\underline{367}, 1098-1107$

Gold R, Arnold DL, Bar-Or A, Hutchinson M, Kappos L, Havrdova E, MacManus DG, Yousry TA, Pozzilli C, Selmaj K et al. (2017): Long-term effects of delayed-release dimethyl fumarate in multiple sclerosis: Interim analysis of ENDORSE, a randomized extension study. Mult Scler 23, $253-265$

Granberg T, Martola J, Kristoffersen-Wiberg M, Aspelin P, Fredrikson S (2013):

Radiologically isolated syndrome--incidental magnetic resonance imaging findings suggestive of multiple sclerosis, a systematic review. Mult Scler 19 , 271-280

Gross CC, Schulte-Mecklenbeck A, Klinsing S, Posevitz-Fejfár A, Wiendl H, Klotz L (2016): Dimethyl fumarate treatment alters circulating T helper cell subsets in multiple sclerosis. Neurol Neuroimmunol Neuroinflamm $\underline{3}$, e183

Grützke B, Hucke S, Gross CC, Herold MVB, Posevitz-Fejfar A, Wildemann BT, Kieseier BC, Dehmel T, Wiendl H, Klotz L (2015): Fingolimod treatment promotes regulatory phenotype and function of B cells. Ann Clin Transl Neurol 2, 119-130

Grytten N, Torkildsen $\varnothing$, Myhr K-M (2015): Time trends in the incidence and prevalence of multiple sclerosis in Norway during eight decades. Acta Neurol Scand 132, 29-36

Hauser SL, Waubant E, Arnold DL, Vollmer T, Antel J, Fox RJ, Bar-Or A, Panzara M, Sarkar N, Agarwal S et al. (2008): B-cell depletion with rituximab in relapsing-remitting multiple sclerosis. N Engl J Med $\underline{358}, 676-688$

Hauser SL, Bar-Or A, Comi G, Giovannoni G, Hartung H-P, Hemmer B, Lublin F, Montalban X, Rammohan KW, Selmaj K et al. (2017): Ocrelizumab versus Interferon Beta-1a in Relapsing Multiple Sclerosis. N Engl J Med 궁, 221-234

Häusser-Kinzel S, Weber MS (2019): The Role of B Cells and Antibodies in Multiple Sclerosis, Neuromyelitis Optica, and Related Disorders. Front Immunol 10, 201

Hein T, Hopfenmüller W (2000): Hochrechnung der Zahl an Multiple Sklerose erkrankten Patienten in Deutschland. Nervenarzt $\underline{71}, 288-294$

Held KD, Epp ER, Clark EP, Biaglow JE (1988): Effect of Dimethyl Fumarate on the Radiation Sensitivity of Mammalian Cells in Vitro. Radiat Res 115, 495-502

Hemmer B, Kerschensteiner M, Korn T (2015): Role of the innate and adaptive immune responses in the course of multiple sclerosis. Lancet Neurol 14, 406-419 
Henderson APD, Barnett MH, Parratt JDE, Prineas JW (2009): Multiple sclerosis: distribution of inflammatory cells in newly forming lesions. Ann Neurol 66, 739-753 Holm Hansen R, Højsgaard Chow H, Sellebjerg F, Rode von Essen M (2018): Dimethyl fumarate therapy suppresses B cell responses and follicular helper T cells in relapsingremitting multiple sclerosis. Mult Scler 25, 1289-1297

Howell OW, Reeves CA, Nicholas R, Carassiti D, Radotra B, Gentleman SM, Serafini B, Aloisi F, Roncaroli F, Magliozzi R et al. (2011): Meningeal inflammation is widespread and linked to cortical pathology in multiple sclerosis. Brain 134, 2755-2771

Howie D (2002): Molecular dissection of the signaling and costimulatory functions of CD150 (SLAM): CD150/SAP binding and CD150-mediated costimulation. Blood 99, 957965

Hunter CA, Jones SA (2015): IL-6 as a keystone cytokine in health and disease. Nat Immunol 16, 448-457

Izquierdo G, Venegas A, Sanabria C, Navarro G (2015): Long-term epidemiology of multiple sclerosis in the Northern Seville District. Acta Neurol Scand 132, 111-117

Jong R de, Bezemer AC, Zomerdijk TP, van de Pouw-Kraan T, Ottenhoff TH, Nibbering PH (1996): Selective stimulation of T helper 2 cytokine responses by the anti-psoriasis agent monomethylfumarate. Eur J Immunol 26, 2067-2074

Juanatey A, Blanco-Garcia L, Tellez N (2018): Ocrelizumab: Eficacia y seguridad en la esclerosis multiple. Rev Neurol 66, 423-433

Kappos L, Gold R, Miller DH, MacManus DG, Havrdova E, Limmroth V, Polman CH, Schmierer K, Yousry TA, Yang M et al. (2008): Efficacy and safety of oral fumarate in patients with relapsing-remitting multiple sclerosis: A multicentre, randomised, doubleblind, placebo-controlled phase IIb study. Lancet $\underline{372}$, 1463-1472

Kesselring J, Beer S (2005): Symptomatic therapy and neurorehabilitation in multiple sclerosis. Lancet Neurol 4, 643-652

van Kester MS, Bouwes Bavinck JN, Quint KD (2015): PML in Patients Treated with Dimethyl Fumarate. N Engl J Med $\underline{373}$, 583-584

Khan F, Turner-Stokes L, Ng L, Kilpatrick T (2007): Multidisciplinary rehabilitation for adults with multiple sclerosis. Cochrane Database Syst Rev, CD006036

Kipnis J (2016): Multifaceted interactions between adaptive immunity and the central nervous system. Science $\underline{353}, 766-771$

Kleinewietfeld M, Manzel A, Titze J, Kvakan H, Yosef N, Linker RA, Muller DN, Hafler DA (2013): Sodium chloride drives autoimmune disease by the induction of pathogenic TH17 cells. Nature $\underline{496}, 518-522$ 
Knippenberg S, Peelen E, Smolders J, Thewissen M, Menheere P, Cohen Tervaert JW, Hupperts R, Damoiseaux J (2011): Reduction in IL-10 producing B cells (Breg) in multiple sclerosis is accompanied by a reduced naïve/memory Breg ratio during a relapse but not in remission. J Neuroimmunol $\underline{239}$, 80-86

Kornberg MD, Bhargava P, Kim PM, Putluri V, Snowman AM, Putluri N, Calabresi PA, Snyder SH (2018): Dimethyl fumarate targets GAPDH and aerobic glycolysis to modulate immunity. Science $\underline{6387}, 449-453$

Krumbholz M, Meinl E (2014): B cells in MS and NMO: pathogenesis and therapy. Semin Immunopathol $\underline{36}, 339-350$

Krumbholz M, Theil D, Derfuss T, Rosenwald A, Schrader F, Monoranu C-M, Kalled SL, Hess DM, Serafini B, Aloisi F et al. (2005): BAFF is produced by astrocytes and upregulated in multiple sclerosis lesions and primary central nervous system lymphoma. J Exp Med 201, 195-200

Kuhlmann T, Lingfeld G, Bitsch A, Schuchardt J, Brück W (2002): Acute axonal damage in multiple sclerosis is most extensive in early disease stages and decreases over time. Brain $\underline{125}, 2202-2212$

Kurosaki T, Kometani K, Ise W (2015): Memory B cells. Nat Rev Immunol 15, 149-159

Kurtzke JF (1983): Rating neurologic impairment in multiple sclerosis An expanded disability status scale (EDSS). Neurology $\underline{33}, 1444$

Lassmann H (2010): Acute disseminated encephalomyelitis and multiple sclerosis. Brain $\underline{133}, 317-319$

Lassmann H, Brück W, Lucchinetti CF (2007): The immunopathology of multiple sclerosis: an overview. Brain Pathol 17, 210-218

Lehmann JCU, Listopad JJ, Rentzsch CU, Igney FH, Bonin A von, Hennekes HH, Asadullah K, Docke W-DF (2007): Dimethylfumarate induces immunosuppression via glutathione depletion and subsequent induction of heme oxygenase 1. J Invest Dermatol 127, 835-845

Leonard W, Donlon T, Lebo R, Greene W (1985): Localization of the gene encoding the human interleukin-2 receptor on chromosome 10. Science 228, 1547-1549

Linker RA, Lee D-H, Ryan S, van Dam AM, Conrad R, Bista P, Zeng W, Hronowsky X, Buko A, Chollate $S$ et al. (2011): Fumaric acid esters exert neuroprotective effects in neuroinflammation via activation of the Nrf2 antioxidant pathway. Brain 134, 678-692 Link H, Huang Y-M (2006): Oligoclonal bands in multiple sclerosis cerebrospinal fluid: an update on methodology and clinical usefulness. J Neuroimmunol 180, 17-28 
Li R, Rezk A, Healy LM, Muirhead G, Prat A, Gommerman JL, Bar-Or A (2015):

Cytokine-Defined B Cell Responses as Therapeutic Targets in Multiple Sclerosis. Front Immunol $\underline{6}, 626$

Li R, Rezk A, Ghadiri M, Luessi F, Zipp F, Li H, Giacomini PS, Antel J, Bar-Or A (2017): Dimethyl Fumarate Treatment Mediates an Anti-Inflammatory Shift in B Cell Subsets of Patients with Multiple Sclerosis. J Immunol 198, 691-698

Litjens NHR, Rademaker M, Ravensbergen B, Rea D, van der Plas MJA, Thio B, Walding A, van Dissel JT, Nibbering PH (2004): Monomethylfumarate affects polarization of monocyte-derived dendritic cells resulting in down-regulated Th1 lymphocyte responses. Eur J Immunol $\underline{34}, 565-575$

Litjens NHR, Rademaker M, Ravensbergen B, Thio HB, van Dissel JT, Nibbering PH (2006): Effects of monomethylfumarate on dendritic cell differentiation. Br J Dermatol $\underline{154}, 211-217$

Liu F, Bardhan K, Yang D, Thangaraju M, Ganapathy V, Waller JL, Liles GB, Lee JR, Liu K (2012): NF- $x$ B directly regulates Fas transcription to modulate Fas-mediated apoptosis and tumor suppression. J Biol Chem 287, 25530-25540

Liu G-Z, Fang L-B, Hjelmström P, Gao X-G (2007): Increased CD8+ central memory T cells in patients with multiple sclerosis. Mult Scler $\underline{13}, 149-155$

Loewe R, Holnthoner W, Groger M, Pillinger M, Gruber F, Mechtcheriakova D, Hofer E, Wolff K, Petzelbauer P (2002): Dimethylfumarate inhibits TNF-induced nuclear entry of NF-kappa B/p65 in human endothelial cells. J Immunol 168, 4781-4787

Longbrake EE, Cross AH (2016): Effect of Multiple Sclerosis Disease-Modifying Therapies on B Cells and Humoral Immunity. JAMA Neurol 73, 219-225

Lowenthal A, van Sande M, Karcher D (1960): The differential diagnosis of neurological diseases by fractionating electro-phorectically the CSF ? J Neurochem $\underline{6}, 51-56$

Lublin FD, Reingold SC, National Multiple Sclerosis Society Advisory Committee on Clinical Trials of New Agents in Multiple (1996): Defining the clinical course of multiple sclerosis Results of an international survey. Neurology $\underline{46}$, 907-911

Lublin FD, Reingold SC, Cohen JA, Cutter GR, Sorensen PS, Thompson AJ, Wolinsky JS, Balcer LJ, Banwell B, Barkhof F et al. (2014): Defining the clinical course of multiple sclerosis: The 2013 revisions. Neurology $\underline{83}$, 278-286

Lucchinetti C, Brück W, Parisi J, Scheithauer B, Rodriguez M, Lassmann H (2000): Heterogeneity of multiple sclerosis lesions: implications for the pathogenesis of demyelination. Ann Neurol 47, 707-717 
Lukasova M, Hanson J, Tunaru S, Offermanns S (2011): Nicotinic acid (niacin): new lipidindependent mechanisms of action and therapeutic potentials. Trends Pharmacol Sci $\underline{32}$, $700-707$

Lund FE (2008): Cytokine-producing B lymphocytes-key regulators of immunity. Curr Opin Immunol 20, 332-338

Lundy SK, Wu Q, Wang Q, Dowling CA, Taitano SH, Mao G, Mao-Draayer Y (2016): Dimethyl fumarate treatment of relapsing-remitting multiple sclerosis influences B-cell subsets. Neurol Neuroimmunol Neuroinflamm $\underline{3}$, e211

Manzel A, Muller DN, Hafler DA, Erdman SE, Linker RA, Kleinewietfeld M (2014): Role of "Western diet" in inflammatory autoimmune diseases. Curr Allergy Asthma Rep $\underline{14}, 404$

Martinelli Boneschi F, Rovaris M, Capra R, Comi G (2005): Mitoxantrone for multiple sclerosis. Cochrane Database Syst Rev, CD002127

McDonald WI, Compston A, Edan G, Goodkin D, Hartung HP, Lublin FD, McFarland HF, Paty DW, Polman CH, Reingold SC et al. (2001): Recommended diagnostic criteria for multiple sclerosis: guidelines from the International Panel on the diagnosis of multiple sclerosis. Ann Neurol 50, 121-127

McGinley MP, Moss BP, Cohen JA (2017): Safety of monoclonal antibodies for the treatment of multiple sclerosis. Expert Opin Drug Saf $\underline{16}$, 89-100

McGuire VA, Ruiz-Zorrilla Diez T, Emmerich CH, Strickson S, Ritorto MS, Sutavani RV, Weibeta A, Houslay KF, Knebel A, Meakin PJ et al. (2016): Dimethyl fumarate blocks proinflammatory cytokine production via inhibition of TLR induced M1 and K63 ubiquitin chain formation. Sci Rep $\underline{6}, 31159$

Medina S, Villarrubia N, La Sainz de Maza S, Lifante J, Costa-Frossard L, Roldán E, Picón C, Álvarez-Cermeño JC, Villar LM (2017): Optimal response to dimethyl fumarate associates in MS with a shift from an inflammatory to a tolerogenic blood cell profile. Mult Scler 24, 1317-1327

Meissner M, Valesky EM, Kippenberger S, Kaufmann R (2012): Dimethyl fumarate - only an anti-psoriatic medication? J Dtsch Dermatol Ges $\underline{10}$, 793-801

Metz I, Traffehn S, Straßburger-Krogias K, Keyvani K, Bergmann M, Nolte K, Weber MS, Bartsch T, Gold R, Brück W (2015): Glial cells express nuclear nrf2 after fumarate treatment for multiple sclerosis and psoriasis. Neurol Neuroimmunol Neuroinflamm $\underline{2}$, e99 Michel L, Touil H, Pikor NB, Gommerman JL, Prat A, Bar-Or A (2015): B Cells in the Multiple Sclerosis Central Nervous System: Trafficking and Contribution to CNSCompartmentalized Inflammation. Front Immunol $\underline{6}, 636$ 
Michell-Robinson MA, Moore CS, Healy LM, Osso LA, Zorko N, Grouza V, Touil H, Poliquin-Lasnier L, Trudelle A-M, Giacomini PS et al. (2016): Effects of fumarates on circulating and CNS myeloid cells in multiple sclerosis. Ann Clin Transl Neurol $\underline{3}, 27-41$

Mikol DD, Barkhof F, Chang P, Coyle PK, Jeffery DR, Schwid SR, Stubinski B, Uitdehaag BMJ (2008): Comparison of subcutaneous interferon beta-1a with glatiramer acetate in patients with relapsing multiple sclerosis (the REbif vs Glatiramer Acetate in Relapsing MS Disease [REGARD] study): A multicentre, randomised, parallel, open-label trial. Lancet Neurol 1, 903-914

Miller D, Barkhof F, Montalban X, Thompson A, Filippi M (2005): Clinically isolated syndromes suggestive of multiple sclerosis, part I: Natural history, pathogenesis, diagnosis, and prognosis. Lancet Neurol 4, 281-288

Miller DH, Leary SM (2007): Primary-progressive multiple sclerosis. Lancet Neurol $\underline{6}$, 903912

Mills EA, Ogrodnik MA, Plave A, Mao-Draayer Y (2018): Emerging Understanding of the Mechanism of Action for Dimethyl Fumarate in the Treatment of Multiple Sclerosis. Front Neurol $\underline{9}, 5$

Milo R, Kahana E (2010): Multiple sclerosis: Geoepidemiology, genetics and the environment. Autoimmun Rev $\underline{9}$, A387-394

Mi S, Hu B, Hahm K, Luo Y, Kam Hui ES, Yuan Q, Wong WM, Wang L, Su H, Chu T-H et al. (2007): LINGO-1 antagonist promotes spinal cord remyelination and axonal integrity in MOG-induced experimental autoimmune encephalomyelitis. Nat Med $\underline{13}, 1228-1233$

Moharregh-Khiabani D, Blank A, Skripuletz T, Miller E, Kotsiari A, Gudi V, Stangel M (2010): Effects of Fumaric Acids on Cuprizone Induced Central Nervous System De- and Remyelination in the Mouse. PLOS ONE $\underline{5}$, e11769

Molnarfi N, Schulze-Topphoff U, Weber MS, Patarroyo JC, Prod'homme T, Varrin-Doyer M, Shetty A, Linington C, Slavin AJ, Hidalgo J et al. (2013): MHC class II-dependent B cell $\mathrm{APC}$ function is required for induction of CNS autoimmunity independent of myelinspecific antibodies. J Exp Med 210, 2921-2937

Montalban X, Gold R, Thompson AJ, Otero-Romero S, Amato MP, Chandraratna D, Clanet M, Comi G, Derfuss T, Fazekas F et al. (2018): ECTRIMS/EAN Guideline on the pharmacological treatment of people with multiple sclerosis. Mult Scler 24, 96-120

Montes Diaz G, Fraussen J, van Wijmeersch B, Hupperts R, Somers V (2018): Dimethyl fumarate induces a persistent change in the composition of the innate and adaptive immune system in multiple sclerosis patients. Sci Rep $\underline{8}, 8194$

Mosser DM, Zhang X (2008): Interleukin-10: new perspectives on an old cytokine. Immunol Rev 226, 205-218 
Nakahara J, Maeda M, Aiso S, Suzuki N (2012): Current Concepts in Multiple Sclerosis: Autoimmunity Versus Oligodendrogliopathy. Clin Rev Allergy Immunol 42, 26-34

Nam S, Lim J-S (2016): Essential role of interferon regulatory factor 4 (IRF4) in immune cell development. Arch Pharm Res $\underline{39}, 1548-1555$

Nieuwkamp DJ, Murk J-L, van Oosten BW, Cremers CHP, Killestein J, Viveen MC, van Hecke W, Frijlink DW, Wattjes MP (2015): PML in a patient without severe lymphocytopenia receiving dimethyl fumarate. N Engl J Med $\underline{372}$, 1474-1476

Nylander A, Hafler DA (2012): Multiple sclerosis. J Clin Invest 122, 1180-1188

Obermeier B, Lovato L, Mentele R, Brück W, Forne I, Imhof A, Lottspeich F, Turk KW, Willis SN, Wekerle H et al. (2011): Related B cell clones that populate the CSF and CNS of patients with multiple sclerosis produce CSF immunoglobulin. J Neuroimmunol 233, 245248

Ontaneda D, Fox RJ (2015): Progressive multiple sclerosis. Curr Opin Neurol 28, 237-243 van Oosten BW, Killestein J, Barkhof F, Polman CH, Wattjes MP (2013): PML in a Patient Treated with Dimethyl Fumarate from a Compounding Pharmacy. N Engl J Med $\underline{368}$, 1658-1659

Orme M, Kerrigan J, Tyas D, Russell N, Nixon R (2007): The effect of disease, functional status, and relapses on the utility of people with multiple sclerosis in the UK. Value Health $\underline{10}, 54-60$

Parodi B, Rossi S, Morando S, Cordano C, Bragoni A, Motta C, Usai C, Wipke BT, Scannevin RH, Mancardi GL et al. (2015): Fumarates modulate microglia activation through a novel HCAR2 signaling pathway and rescue synaptic dysregulation in inflamed CNS. Acta Neuropathol 130, 279-295

Paty DW, Li DK (1993): Interferon beta-1b is effective in relapsing-remitting multiple sclerosis. II. MRI analysis results of a multicenter, randomized, double-blind, placebocontrolled trial. UBC MS/MRI Study Group and the IFNB Multiple Sclerosis Study Group. Neurology $\underline{43}$, 662-667

Pierrot-Deseilligny C, Souberbielle J-C (2013): Contribution of vitamin D insufficiency to the pathogenesis of multiple sclerosis. Ther Adv Neurol Disord $\underline{6}, 81-116$

Pittock SJ, Rodriguez M: Benign Multiple Sclerosis: A Distinct Clinical Entity with Therapeutic Implications; In: Advances in multiple sclerosis and experimental demyelinating diseases, edited by Rodriguez M. (Current topics in microbiology and immunology v. 318): Springer. Berlin 2008, 1-17

Polman CH, Reingold SC, Edan G, Filippi M, Hartung H-P, Kappos L, Lublin FD, Metz LM, McFarland HF, O'Connor PW et al. (2005): Diagnostic criteria for multiple sclerosis: 2005 revisions to the "McDonald Criteria". Ann Neurol 모, 840-846 
Polman CH, Reingold SC, Banwell B, Clanet M, Cohen JA, Filippi M, Fujihara K, Havrdova E, Hutchinson M, Kappos L et al. (2011): Diagnostic criteria for multiple sclerosis: 2010 revisions to the McDonald criteria. Ann Neurol 69, 292-302

Prasad KV, Ao Z, Yoon Y, Wu MX, Rizk M, Jacquot S, Schlossman SF (1997): CD27, a member of the tumor necrosis factor receptor family, induces apoptosis and binds to Siva, a proapoptotic protein. Proc Natl Acad Sci U S A 모, 6346-6351

Pype S, Declercq W, Ibrahimi A, Michiels C, van Rietschoten JG, Dewulf N, Boer M de, Vandenabeele P, Huylebroeck D, Remacle JE (2000): TTRAP, a novel protein that associates with CD40, tumor necrosis factor (TNF) receptor-75 and TNF receptorassociated factors (TRAFs), and that inhibits nuclear factor-kappa B activation. J Biol Chem $\underline{275}, 18586-18593$

Raffel J, Wallace A, Gveric D, Reynolds R, Friede T, Nicholas R (2017): Patient-reported outcomes and survival in multiple sclerosis: A 10-year retrospective cohort study using the Multiple Sclerosis Impact Scale-29. PLoS Med 14, e1002346

Reich K, Thaci D, Mrowietz U, Kamps A, Neureither M, Luger T (2009): Efficacy and safety of fumaric acid esters in the long-term treatment of psoriasis--a retrospective study (FUTURE). J Dtsch Dermatol Ges $\underline{7}, 603-611$

Reick C, Ellrichmann G, Thöne J, Scannevin RH, Saft C, Linker RA, Gold R (2014): Neuroprotective dimethyl fumarate synergizes with immunomodulatory interferon beta to provide enhanced axon protection in autoimmune neuroinflammation. Exp Neurol 257, $50-56$

Rosenkranz T, Novas M, Terborg C (2015): PML in a Patient with Lymphocytopenia Treated with Dimethyl Fumarate. N Engl J Med $\underline{372}$, 1476-1478

Rovaris M, Confavreux C, Furlan R, Kappos L, Comi G, Filippi M (2006): Secondary progressive multiple sclerosis: Current knowledge and future challenges. Lancet Neurol $\underline{5}$, $343-354$

Scannevin RH, Chollate S, Jung M-y, Shackett M, Patel H, Bista P, Zeng W, Ryan S, Yamamoto M, Lukashev M et al. (2012): Fumarates promote cytoprotection of central nervous system cells against oxidative stress via the nuclear factor (erythroid-derived 2)-like 2 pathway. J Pharmacol Exp Ther 341, 274-284

Schimrigk S, Brune N, Hellwig K, Lukas C, Bellenberg B, Rieks M, Hoffmann V, Pöhlau D, Przuntek H (2006): Oral fumaric acid esters for the treatment of active multiple sclerosis: an open-label, baseline-controlled pilot study. Eur J Neurol 13, 604-610

Schmidt TJ, Ak M, Mrowietz U (2007): Reactivity of dimethyl fumarate and methylhydrogen fumarate towards glutathione and $\mathrm{N}$-acetyl-L-cysteine--preparation of Ssubstituted thiosuccinic acid esters. Bioorg Med Chem 15, 333-342 
Schulze-Topphoff U, Varrin-Doyer M, Pekarek K, Spencer CM, Shetty A, Sagan SA, Cree BAC, Sobel RA, Wipke BT, Steinman L et al. (2016): Dimethyl fumarate treatment induces adaptive and innate immune modulation independent of Nrf2. Proc Natl Acad Sci U S A $\underline{113}, 4777-4782$

Schweckendieck W (1959): Heilung von Psoriasis vulgaris. Med Monatsschr 13, 103-104 Scott LJ, Figgitt DP (2004): Mitoxantrone: a review of its use in multiple sclerosis. CNS Drugs 18, 379-396

Sedal L, Winkel A, Laing J, Law LY, McDonald E (2017): Current concepts in multiple sclerosis therapy. Degener Neurol Neuromuscul Dis $7,109-125$

Simpson S, Blizzard L, Otahal P, van der Mei I, Taylor B (2011): Latitude is significantly associated with the prevalence of multiple sclerosis: a meta-analysis. J Neurol Neurosurg Psychiatr $\underline{82}, 1132-1141$

Smith MD, Martin KA, Calabresi PA, Bhargava P (2017): Dimethyl fumarate alters B-cell memory and cytokine production in MS patients. Ann Clin Transl Neurol 4, 351-355

Sospedra M, Martin R (2005): Immunology of multiple sclerosis. Annu Rev Immunol $2 \underline{3}$, 683-747

Spencer CM, Crabtree-Hartman EC, Lehmann-Horn K, Cree BAC, Zamvil SS (2015): Reduction of CD8(+) T lymphocytes in multiple sclerosis patients treated with dimethyl fumarate. Neurol Neuroimmunol Neuroinflamm 2, e76

Stadelmann C, Brück W (2004): Lessons from the neuropathology of atypical forms of multiple sclerosis. Neurol Sci $\underline{25}$, s319-s322

Stys PK, Zamponi GW, van Minnen J, Geurts JJG (2012): Will the real multiple sclerosis please stand up? Nat Rev Neurosci $\underline{13}, 507-514$

Tahvili S, Zandieh B, Amirghofran Z (2015): The effect of dimethyl fumarate on gene expression and the level of cytokines related to different $T$ helper cell subsets in peripheral blood mononuclear cells of patients with psoriasis. Int J Dermatol $\underline{54}$, e254-260

Thiessen A, Schmidt MM, Dringen R (2010): Fumaric acid dialkyl esters deprive cultured rat oligodendroglial cells of glutathione and upregulate the expression of heme oxygenase 1. Neurosci Lett $\underline{475}, 56-60$

Thompson AJ, Polman CH, Miller DH, McDonald WI, Brochet B, Filippi M Montalban X, Sá J de (1997): Primary progressive multiple sclerosis. Brain 120, 1085-1096

Thompson AJ, Banwell BL, Barkhof F, Carroll WM, Coetzee T, Comi G, Correale J, Fazekas F, Filippi M, Freedman MS et al. (2018): Diagnosis of multiple sclerosis: 2017 revisions of the McDonald criteria. Lancet Neurol 17, 162-173 
Torkildsen O, Brunborg LA, Myhr K-M, Bø L (2008): The cuprizone model for demyelination. Acta Neurol Scand Suppl 188, 72-76

Traub J, Traffehn S, Ochs J, Häusser-Kinzel S, Stephan S, Scannevin R, Brück W, Metz I, Weber MS (2019): Dimethyl fumarate impairs differentiated B cells and fosters central nervous system integrity in treatment of multiple sclerosis. Brain Pathol 29, $640-657$

Treumer F, Zhu K, Glaser R, Mrowietz U (2003): Dimethylfumarate is a potent inducer of apoptosis in human T cells. J Invest Dermatol 121, 1383-1388

Vargas DL, Tyor WR (2017): Update on disease-modifying therapies for multiple sclerosis. J Investig Med 모, 883-891

Wallbrecht K, Drick N, Hund A-C, Schön MP (2011): Downregulation of endothelial adhesion molecules by dimethylfumarate, but not monomethylfumarate, and impairment of dynamic lymphocyte-endothelial cell interactions. Exp Dermatol 20, 980-985

Wang Q, Chuikov S, Taitano S, Wu Q, Rastogi A, Tuck SJ, Corey JM, Lundy SK, MaoDraayer Y (2015): Dimethyl Fumarate Protects Neural Stem/Progenitor Cells and Neurons from Oxidative Damage through Nrf2-ERK1/2 MAPK Pathway. Int J Mol Sci 16, 1388513907

Weinshenker BG (1998): The natural history of multiple sclerosis: Update 1998. Semin Neurol 18, 301-307

Wilms H, Sievers J, Rickert U, Rostami-Yazdi M, Mrowietz U, Lucius R (2010):

Dimethylfumarate inhibits microglial and astrocytic inflammation by suppressing the synthesis of nitric oxide, IL-1beta, TNF-alpha and IL-6 in an in-vitro model of brain inflammation. J Neuroinflammation $\underline{7}, 30$

Wucherpfennig KW, Catz I, Hausmann S, Strominger JL, Steinman L, Warren KG (1997): Recognition of the immunodominant myelin basic protein peptide by autoantibodies and HLA-DR2-restricted T cell clones from multiple sclerosis patients. Identity of key contact residues in the B-cell and T-cell epitopes. J Clin Invest 100, 1114-1122

Wu Q, Wang Q, Mao G, Dowling CA, Lundy SK, Mao-Draayer Y (2017): Dimethyl Fumarate Selectively Reduces Memory T Cells and Shifts the Balance between Th1/Th17 and Th2 in Multiple Sclerosis Patients. J Immunol 198, 3069-3080

Wynn D, Kaufman M, Montalban X, Vollmer T, Simon J, Elkins J, O'Neill G, Neyer L, Sheridan J, Wang C et al. (2010): Daclizumab in active relapsing multiple sclerosis (CHOICE study): A phase 2, randomised, double-blind, placebo-controlled, add-on trial with interferon beta. Lancet Neurol $\underline{9}$, 381-390

Zhu K-J, Zhou W-F, Zhou K, Zheng M, Ulrich M (2002): Effects of fumaric acid esters on differentiation of dendritic cells in vitro. Zhejiang Da Xue Xue Bao Yi Xue Ban $\underline{31}$, 453456 
